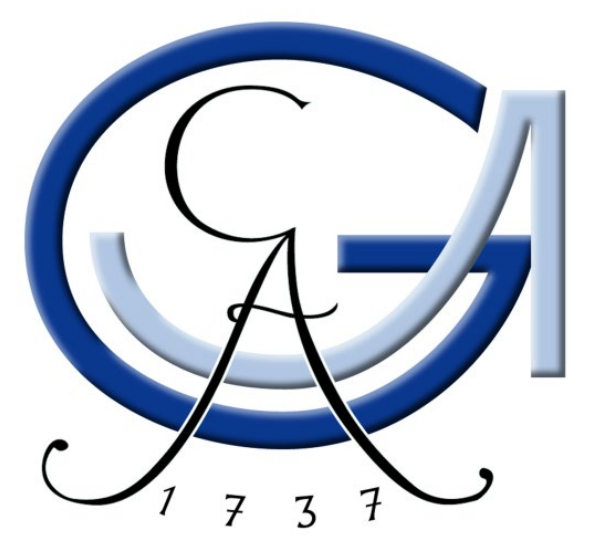

\title{
Integration of autonomous and pattern recognition controls in hand prostheses
}

\author{
Dissertation \\ for the award of the degree \\ "Doctor rerum naturalium" \\ of the Georg-August-Universität Göttingen
}

Within the doctoral program Computer Science (PCS) of the Georg-August University School of Science (GAUSS)

Submitted by Jérémy Manual Jacques MOUCHOUX From Saint Cyr l'École, FRANCE

Göttingen, 2021 


\section{Thesis Committee}

First supervisor: Prof. Dr. Florentin Wörgötter

Third Institute of Physics

Georg-August-Universität Göttingen

Second Supervisor: Prof. Dr. Arndt F. Schilling

Department for Trauma Surgery, Orthopedics, and Plastic Surgery

University Medical Center Göttingen

Third Supervisor: Dr. Marko Markovic

Department for Trauma Surgery, Orthopedics, and Plastic Surgery

University Medical Center Göttingen

Members of the Examination Board:

Reviewer: Prof. Dr. Florentin Wörgötter

Second Reviewer: Prof. Dr. Arndt F. Schilling

Further members of the Examination Board:

Prof. Dr. Marcus Baum

Prof. Dr. Alexander Ecker

Prof. Dr. David Liebetanz

Prof. Dr. Strahinja Dosen

Date of the oral examination: $19^{\text {th }}$ November 2021 


\section{Declaration}

This dissertation results from my own work and includes no outcome of work done in collaboration except where specifically indicated in the text. It has not been previously submitted, in part or whole, to any university or institution for any degree, diploma, or other qualification.

This work was funded by the German Ministry for Education and Research (BMBF) under the project INOPRO 16SV7657.

Hereby, I declare that I have written this thesis independently and with no other aids and sources than quoted. 


\section{Abstract}

Thanks to the progress in the robotic and mechatronic fields, upper limb prostheses became more and more dexterous, getting closer to closing the gap between the natural limb and the medical device replacing it. However, the standard prosthesis controller spread on the market is still limited in the number of functions it can efficiently control and constitute a bottleneck in prosthesis use. Two leading solutions are currently under research to tackle this discrepancy: machine learning algorithms and autonomous controllers. Myocontrollers based on machine learning algorithms enable the user to control a high but still limited number of functions directly. On the other hand, autonomous controllers are based on a diversification of the sensor modalities to integrate the context and the user's intention in control and automatise part of the grasping process, relieving the user from the physical and potentially cognitive workload associated with it. This thesis focuses on the impact of the combination of these two solutions. Therefore, two studies investigated the gain of performance gain and the range of applicability of an association of a semiautonomous system to a machine learning myo-controller for upper limb prostheses.

This dissertation introduces first a method to preshape the prosthesis based on the prediction of the user's intended grasping strategy. This method system supports the user in real-time by preshaping the prosthetic device's hand and wrist during a reaching phase of a prehensile action. This result is achieved by merging data from inertial measurement units, computer vision, and positions and pressure sensors to reproduce artificial proprioception, artificial exteroception, and short-term memory. The autonomous controller developed was designed to support the user in dynamic objects-crowded conditions.

In a second phase, it has been assessed whether a semi-autonomous system associated with a machine learning myo-controller could improve the 
performance compared to the same machine learning controller alone. To assess this system, eight able-bodied and two amputee participants performed a newly developed test featuring a sequence of re-localisation tasks in a scene with multiple objects. The two amputee participants completed a standard rehabilitation test as well. The results revealed that the semi-autonomous system increased the time performance and reduced the physical effort for the total duration of the trial, and more specifically, in the preshaping phase of the task. The semi-autonomous system also reduced the need to control the prosthetic wrist manually.

Eventually, the last part of this dissertation focuses on the interactions between the autonomous controller and the user. It investigates the impact, through the addition of artificial error on the autonomous system output, of different shared control modalities which define the application, according to the user's commands, of the decisions of the autonomous controller on the prosthesis. Ten able-bodied participants performed a dual-task combining a reach-and-grasp task and an auditory reaction task. Time performance, physical and cognitive workload were recorded. This test implemented different controlsharing modalities at different levels of error. The results revealed that the shared control modalities significantly impact the task performance and the physical effort required to complete the task. The effect of the level of added errors on the three different outcomes varies between the control-sharing modalities. These results, therefore, provide valuable information to design and compare semi-autonomous upper limb prosthesis systems.

In conclusion, by demonstrating the benefit of semi-autonomous systems and the investigation of their application conditions, this thesis advances the development of a new generation of prosthesis controllers that automatize parts of the actuation of the prosthesis. This research enlarges the current control bottleneck, which prevents prosthesis users from taking full advantage of their highly developed device. Therefore, it can contribute to increasing the support and the autonomy that upper limb prostheses can provide to their users in daily life. 


\section{Acknowledgements}

First, I would like to thank my first supervisor, Prof. Dr. Florentin Wörgötter, for his general view on the project, which definitely influenced my work.

I would also like to express my thankfulness to Prof. Dr. Arndt F. Schilling for his implication in this project, the always valuable advice, and the management of the whole laboratory.

I would like to sincerely thank Dr. Marko Markovic for his close supervision, availability during this thesis, and technical support with the laboratory equipment.

Moreover, I thank the whole Applied Rehabilitation Technology Laboratory for the very nice and always helpful ambience. Especially, I would like to thank Frau Modrok, who has always been helping and caring when I struggled with the administration or when I arrived in Göttingen.

Further, I thank all the subjects for their participation, their patience, and their feedback.

My last thanks are to my family for the patience, motivation, and support they provided me.

Thanks, Covid-19. Without you, it would have been much less challenging. 


\section{Content}

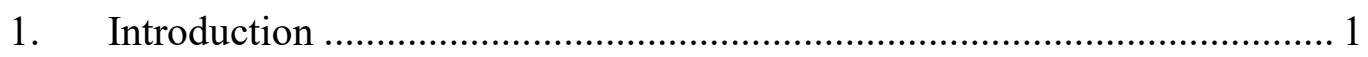

1.1. Treatment solutions for upper hand deficiency .................................... 3

1.1.1. Surgical treatment: Hand transplantation ............................................. 3

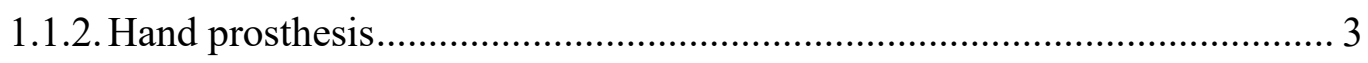

1.2. The different myocontrol implementations ............................................. 7

1.2.1. The historical "two-channels control" .................................................. 7

1.2.2. Pattern recognition-based myo-control .................................................. 9

1.3. Technical solutions to improve manual control .................................... 11

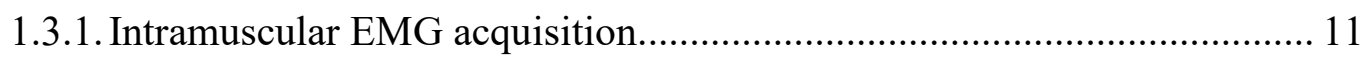

1.3.2. Diversifying the sensor modalities ...................................................... 12

1.3.3. Using other modalities to control the prosthesis .................................... 13

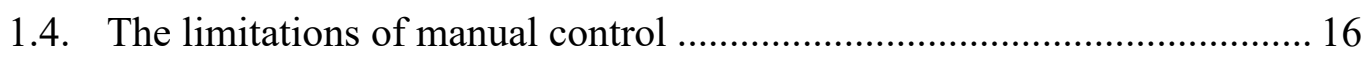

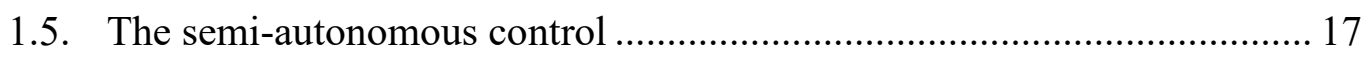

1.5.1. Intention prediction and associated automation ................................... 19

1.5.2. The rules governing the semi-autonomous system operation .................. 26

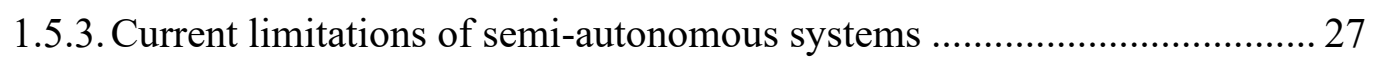

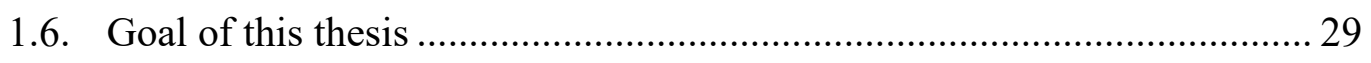

2. Automatization of the wrist and the hand based on the prediction of the intended object's grasped side............................................................. 31

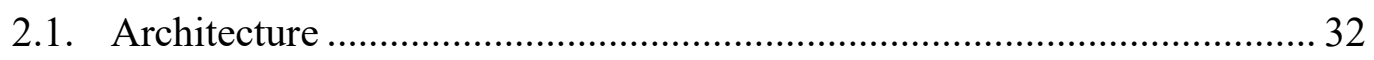

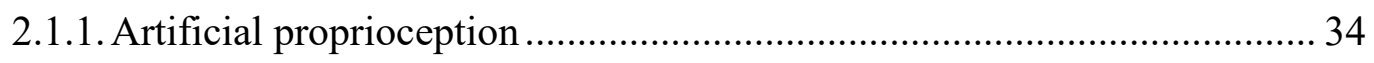

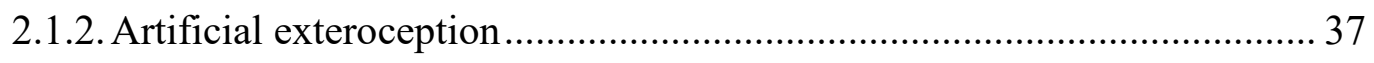


2.1.3. Scene generation: short-term memory .................................................. 41

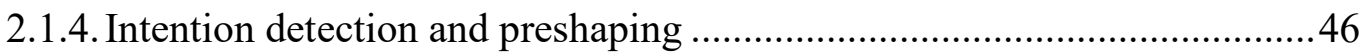

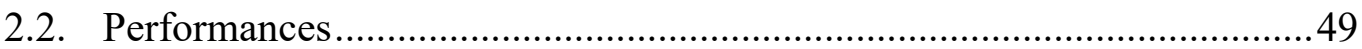

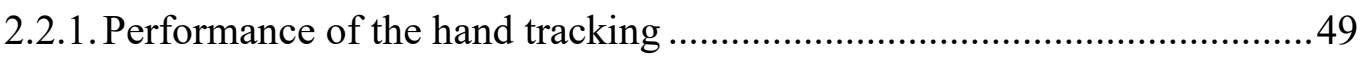

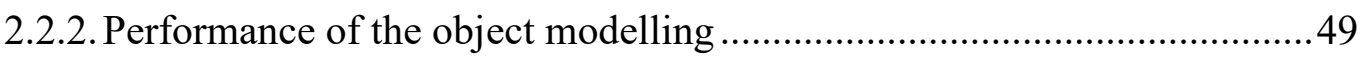

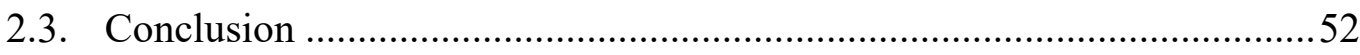

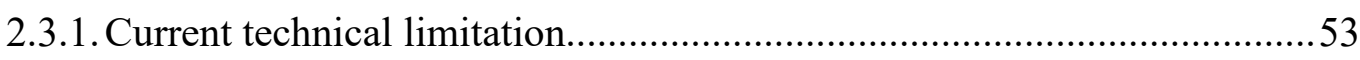

3. Assessing the support provided by an autonomous controller when combined with an ML controller..............................................................55

3.1. Implemented Human Machine Interface ...............................................56

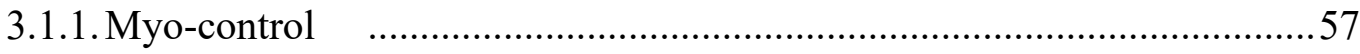

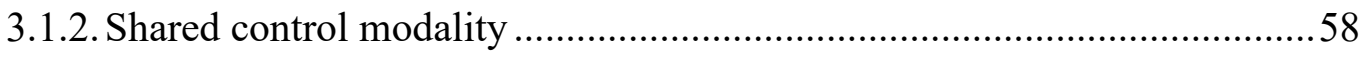

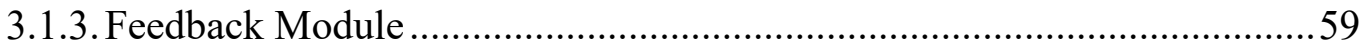

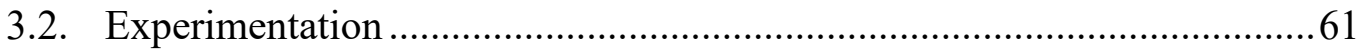

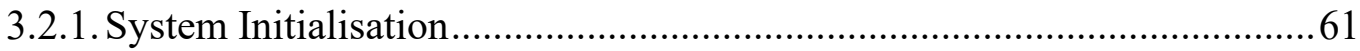

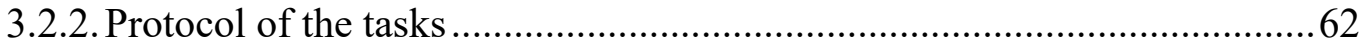

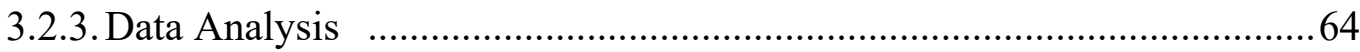

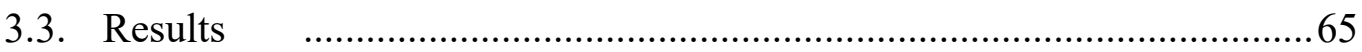

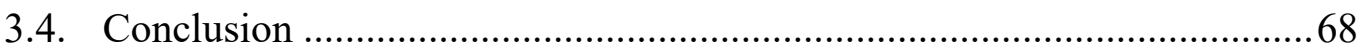

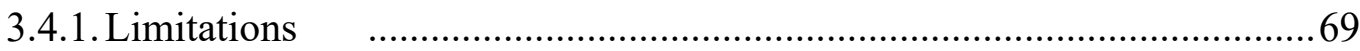

4. Impact of the sharing control modality on the user's performance and

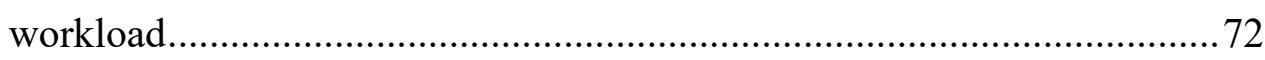

4.1. A "Wizard-of-Oz" study design........................................................ 74

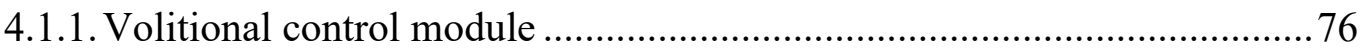

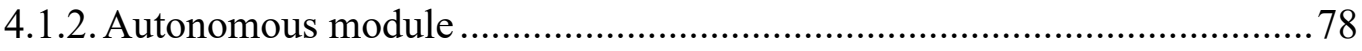

4.2. Implementation of the different shared control modalities ........................ 82 


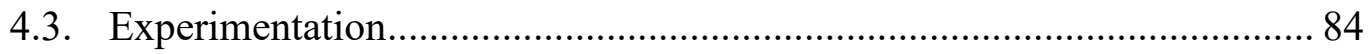

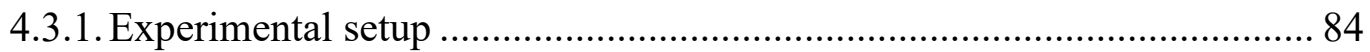

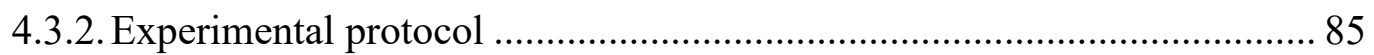

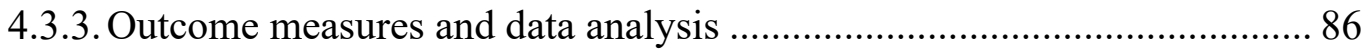

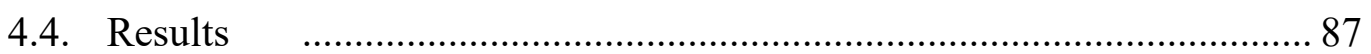

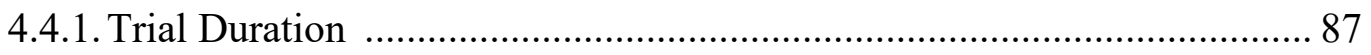

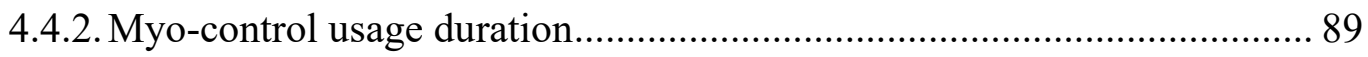

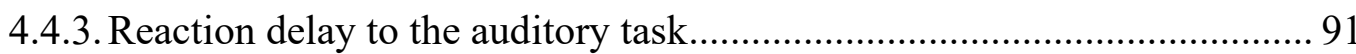

4.5. Representative examples of user's behaviour in continuous, simultaneous,

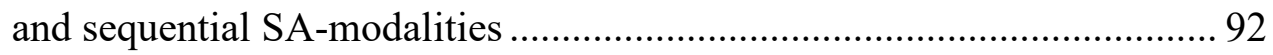

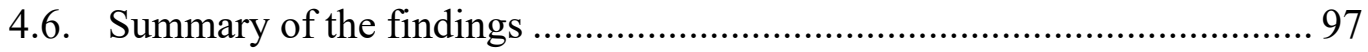

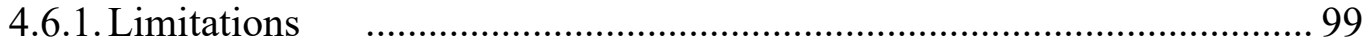

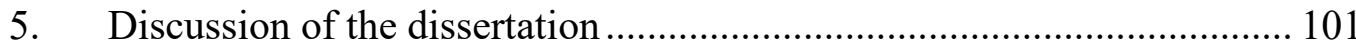

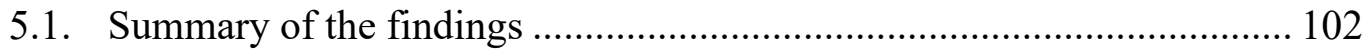

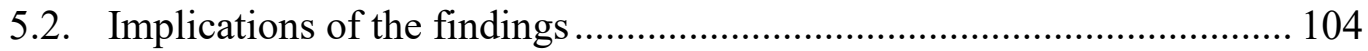

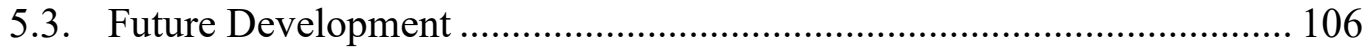




\section{Abbreviations}

ADLs

AR

DoFs

HMI

IMU

LCCP

LDA

ML

PCL

RANSAC

SA

SHAP

SoA

VCCS
Activities of Daily Living

Augmented Reality

Degrees of Freedom

Human Machine Interface

Inertial Measurement Unit

Locally Convex Connected Patches

Linear Discriminant Analysis

Machine Learning

Point Cloud Library

Random sample consensus

Semi-Autonomous

Southampton Hand Assessment Procedure

State-of-the-Art

Voxel Cloud Connectivity Segmentation 


\section{List of figures}

Figure 1.1 Illustration of the different levels of amputations with the associated number of lost DoFs

Figure 1.2 Illustration of a transplanted hand.............................................. 3

Figure 1.3 Illustration of a body-powered prosthesis ..................................... 4

Figure 1.4 Functioning of a myoelectric prosthesis.......................................... 5

Figure 1.5 Illustration of the 2-channel control............................................. 7

Figure 1.6 Scheme of a Pattern recognition based myo-control........................ 9

Figure 1.7 Illustration of the use of another modality to control one functionality

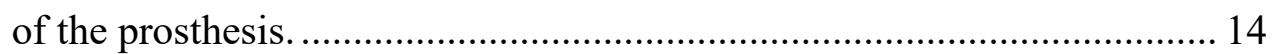

Figure 1.8 Illustration of the limitations of Manual control........................... 16

Figure 1.9 Scheme of functioning of a semi-autonomous system.................. 18

Figure 2.1 Photography of the material used with highlight of the components used for the artificial proprioception module. ......................................... 34

Figure 2.2 Scheme of the computer vision processing.................................. 38

Figure 2.3 Comparison between the real scene and the generated scene. ........ 41

Figure 2.4. Enumeration of the different possible updates coming from the

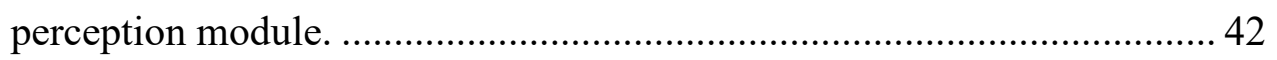

Figure 2.5 Flowchart of the shape parliament algorithm............................. 45

Figure 2.6. Illustration of the selection's areas........................................... 47

Figure 2.7 Acquired objects position during the assessment of the object modelling module performances. ........................................................... 50

Figure 2.8 Deviation to the average position for different objects at five different

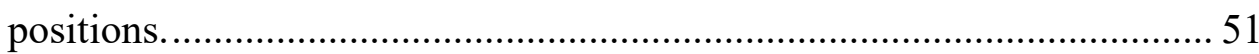


Figure 2.9 Acquired objects size during the assessment of the object modelling module

Figure 3.1 Photography of the material used during the experiment with the

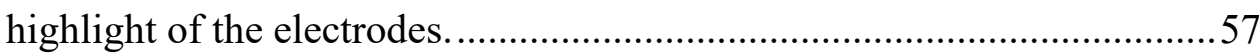

Figure 3.2 Scheme of the control part of the SA system................................58

Figure 3.3 Photography of the components used with highlight of the AR

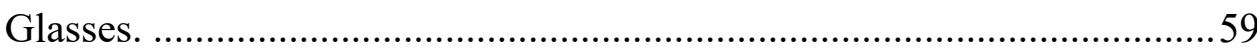

Figure 3.4 Illustration of the process of a grasp with detail of the different phases and representation of the feedback provided to the user through AR glasses

Figure 3.5 Overview of the Cluttered scene interaction test (CSI) 63

Figure 3.6 Trial time decomposition for the cluttered scene interaction (CSI) test performed by both able participants (A) and by amputee participants (B).

Figure 3.7 Performance of the two amputee subjects during the SHAP tests...67

Figure 4.1 Schema of the SA system replicated in a controlled environment .. 76

Figure 4.2 Sensorized prosthesis adaptor 77

Figure 4.3 Decomposition of the different phases of the automatic preshaping. .79

Figure 4.4 Scheme of the different shared control modalities implemented. ...82

Figure 4.5 Illustration of the experimental setup. .84

Figure 4.6 Outcomes of the duration of the trial during the different conditions.

Figure 4.7 Outcomes of the duration of the myocontrol usage during the different conditions. .89

Figure 4.8 Outcomes of the reaction delay during the different conditions......91

Figure 4.9 Examples of the behaviour of the pose of the three DoFs of the prosthesis under manual condition. .92 
Figure 4.10 Examples of the behaviour of the pose of the three DoFs of the prosthesis under the no error condition for the three shared control modalities.

Figure 4.11 Examples of the pose behaviour of the prosthesis's three DoFs under the $30^{\circ}$ error condition for the three shared control modalities. 95

Figure 4.12 Examples of the pose behaviour of the prosthesis's three DoFs under the $60^{\circ}$ error condition for the three shared control modalities. 96 


\section{Introduction}

Our hands are one of the most important tools we have to interact with the world. Within a (life-)long process, we learn and improve how to control and use them with increasing precision and accuracy. For this reason, losing one of them has a major impact on the motor system and the interaction abilities that one has with the objects surrounding us. It has been estimated that in 2005, 541.000 persons were living with an upper hand amputation in the United States [1]. Other countries such as Italy and the United Kingdom report 3500 and 5200 upper limb amputations every year [2]. The first cause of upper limb amputation is traumatism, followed by neoplasia and vascular and infectious diseases, as stated by [3] (Frontera \& Silver, 2004 (cited in Cordella et al., 2016).

Depending on the level of amputation, the individual can lose a different number of functionalities, summarised in Figure 1.1. When taking a hand model with 19 Degrees of Freedom (DoFs) [4], a transradial amputation, depraving the individual from the limb below the elbow, leads to losing up to 22 natural DoFs.

Such amputations have repercussions on the whole body. Patients do not only lose an excellent tool to interact with their environment, but they also suffer from the unbalance caused by the ablation of the upper limb extremity. Indeed, it has been 
shown that Upper limb amputees have more Musculoskeletal complaints [5]. In unilateral amputation, the healthy arm can also get affected by overuse injuries due to over-reliance on it [6]-[8]. Similar impairments can also occur for people with a congenital hand deficiency, with an incidence of 5.25 per 10,000 live births [9].

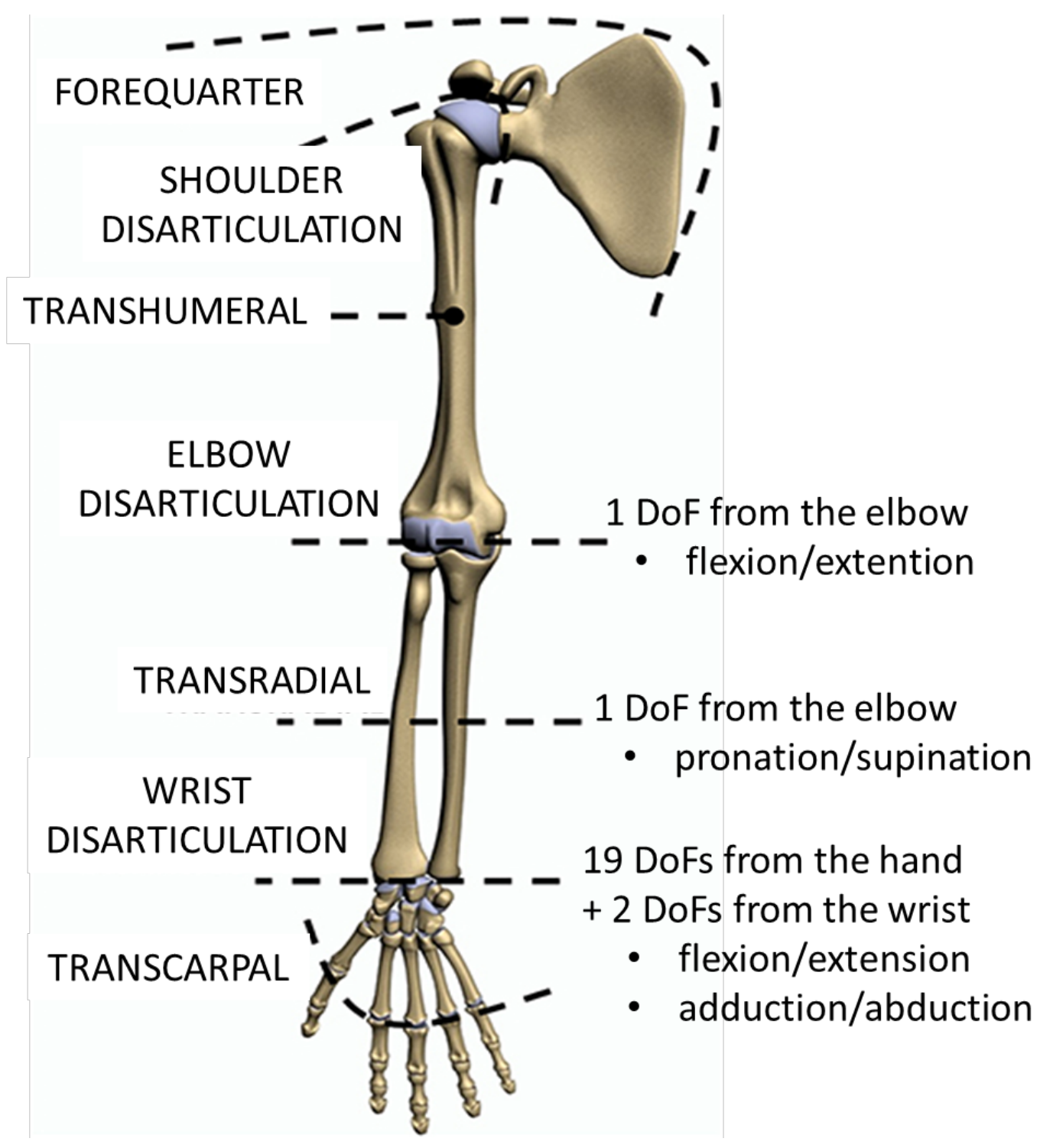

Figure 1.1 Illustration of the different levels of amputations with the associated number of lost DoFs Adapted with permission from [2] 


\subsection{Treatment solutions for upper hand deficiency}

The following section provides an overview of different approaches that have been developed, up to now, to provide help for people with upper limb deficiency to (re-)gain possibilities of self-reliant interaction abilities with their environments.

\subsubsection{Surgical treatment: Hand transplantation}
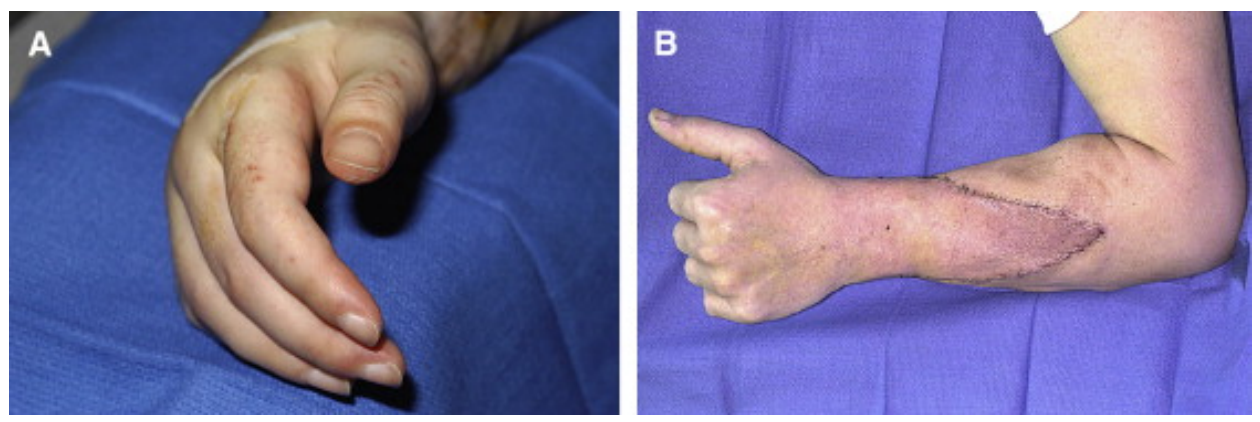

Figure 1.2 Illustration of a transplanted hand. A) shows a swelling and rash hand during an acute rejection. B) shows a hand from another patient after six weeks. Used with permission from [10]

To provide a solution to improve the life of amputees, hand transplantation has been developed since 1998 [11]. During this procedure, a hand is taken from a compatible donor and grafted on the individual. The acceptance of the new hand by the patient's body requires immunosuppressants for the rest of his/her life. A recent review [12] reports 113 transplants performed on 76 patients worldwide. The cost of such a procedure, the graft rejection rate of $9.5 \%$, and the high risks associated with immunosuppression are the main reasons for the relatively low number of transplants compared to the number of amputations.

The other way to help patients with a missing limb is to provide an artificial replacement, called a prosthesis. Already existing for at least 3000 years [13], prostheses have continued to develop and become more complex to resemble the appearance of the original limb and restore more of the lost functionality. With regard to the upper limb prostheses, several types of prostheses emerged according to their actuation. 


\section{I) $\quad$ Passive prostheses}

Esthetic prostheses, often called "cosmesis", are passive prostheses mainly aiming to replace the visual appearance of the missing limb. While passive, some designs allow some functionalities using springs or bending materials to support the user. The knight hands with the famous example of the hand of Götz von Berlichingen [14], a Franconian imperial knight in the $16^{\text {th }}$ century known to be able to hold his sword with his prosthesis thanks to metal slides acting as springs. Thus this prosthesis offered a thumb with one degree of freedom (DoF) mechanically actuated. Passive prostheses with mechanical actuation are still developed, such as the Delft SelfGrasping Hand using the wrist angle to open and close the hand [15], which contributes to answering their apparent underdevelopment [16].

\section{II) Body powered}

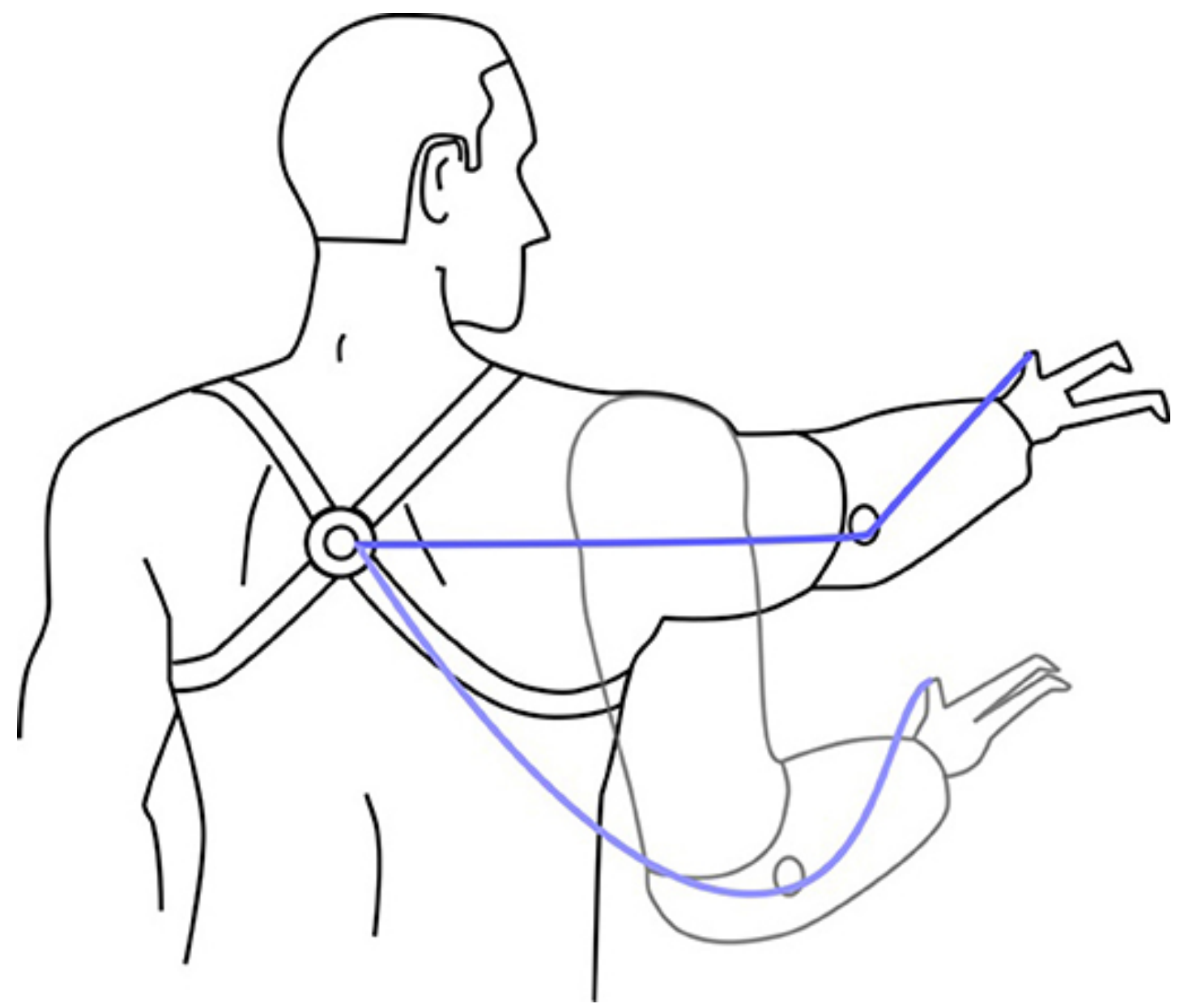

Figure 1.3 Illustration of a body-powered prosthesis , used with permission from [17] 


\section{Chapter 1: Introduction}

Body-powered prostheses are active prostheses, able to perform some movements like opening-closing, entirely powered by the remaining muscles of the user, potentially dedicated to other functionalities of the body. They were greatly developed during the early 20th century [18]. A century later, they still equip $30 \%$ of the upper limb deficient population [19]. The standard model uses a cable pulled between the shoulders to actuate the prosthesis [20]. Other models use the wrist or the elbow to control the aperture of the finger [21], [22], according to the joints available.

While they appear technologically less developed than myoelectric prostheses, the feedback provided by the cable's tension and the simplicity of the device actuation make body-powered prostheses better in durability, training time, frequency of adjustment, maintenance, and feedback, according to [23]. It was also a body-powered prosthesis user who won the Cybathlon 2016, a competition comparing the completion time of different tasks reproducing daily life activities [24].

Despite these good performances, body-powered prostheses are being overlooked because of the better appearance of myoelectric prostheses, slower movement, insufficient grasp strength, increased mass, and energy expenditure/consumption for operation.

\section{III) $\underline{\text { Myoelectric }}$}
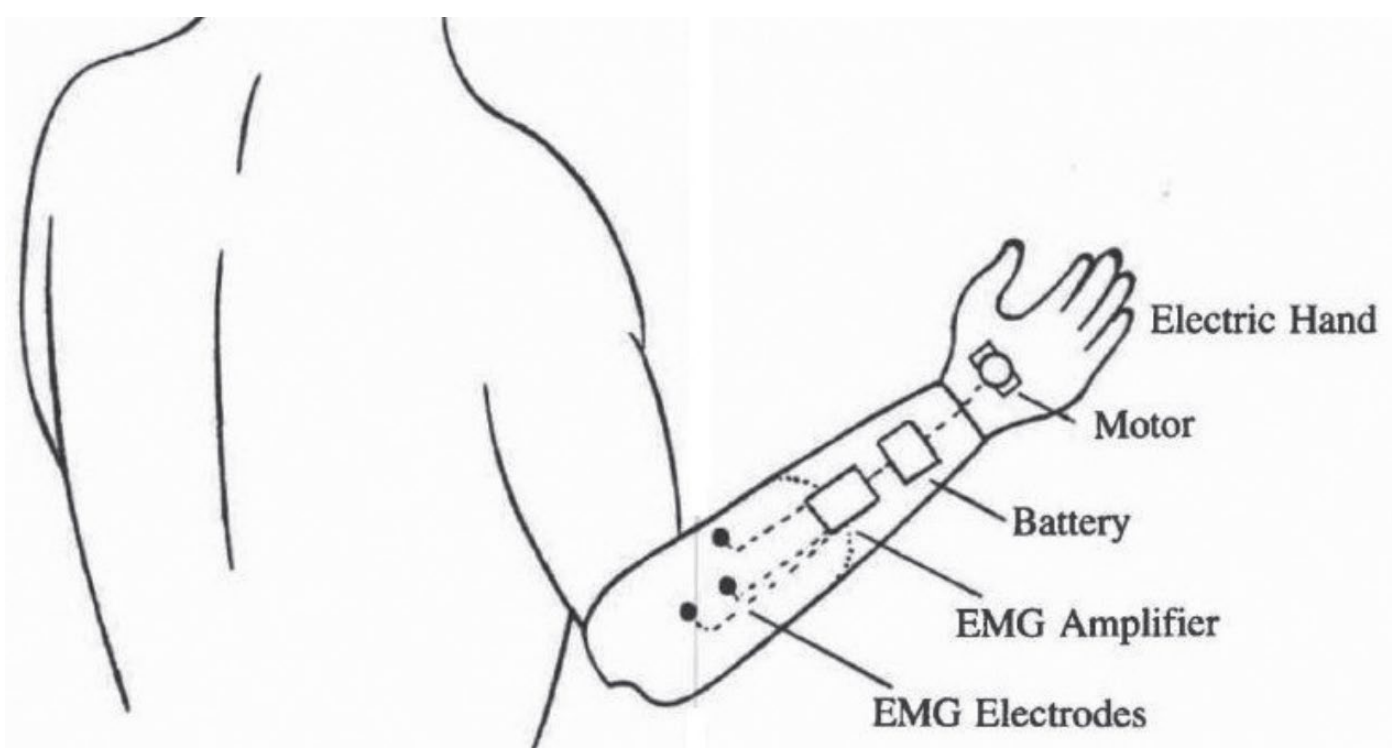

Figure 1.4 Functioning of a myoelectric prosthesis, used with permission from [25] 


\section{Chapter 1: Introduction}

On the other hand, myoelectric prostheses are actuated by battery-operated motors. The support provided by the engine relieves the user of applying and maintaining tension to hold an object. The actuation of these prostheses is controlled by an interface allowing the interaction of the human and the prostheses (humanmachine Interface, HMI). The original HMI was based on detecting the contractions of the user's remaining muscles using electromyogram signals to trigger the actuation of the prosthesis. The control of the prosthesis by muscle contractions is called myocontrol. These prostheses currently equip 36\% of the upper limb prosthesis users population [19].Today, myoelectric hand prostheses are diversifying. The first models, such as those from Reinhold Reiter in 1948 [26], Alexander Kobrinski in 1960 [27], or Miodrag Rakic in 1957-1962 [28], had only one DoF actuating all the fingers together. To mimic the flexibility of the human hand, the number of different possible hand actuation was increased. Firstly, the thumb was given an extra DoF, thus diversifying the number of grasp types. Secondly, the fingers were independently actuated, increasing the number of grasps of the prosthetic hand to 20 in 2015 [29]. This increase in the number of grasps brings the dexterity of myoelectric prostheses closer to that of the human hand, which can perform 33 possible grasps [30].

Another mechanical design offers independent fingers but with fewer actuators. This design, called underactuation, enables the fingers to adapt to the object's shape or even to the external constraints such as the table's surface when grasping the object [31], [32]. The trade-off of under-actuation is the reduced possibility to activate a specific finger scheme.

Despite a high dexterity, a lack of wrist rotation causes compensatory movement, damaging the body in the long term [33], [34]. Therefore, in addition to the hand, active prosthetic wrists have been developed and are currently available. They provide either active rotation, flexion, or both [35].

Overall, the comparison between new prosthetic devices and a natural hand shows that, thanks to the improved technology, the capabilities of hand prostheses have come closer to the mechanical capabilities of a natural hand. The combination of the mechanical improvement with a particular focus on the most used grasps has 
further increased the usability of the prostheses. However, this also shows that there is still room for improvement.

\subsection{The different myocontrol implementations}

\subsubsection{The historical "two-channels control"}

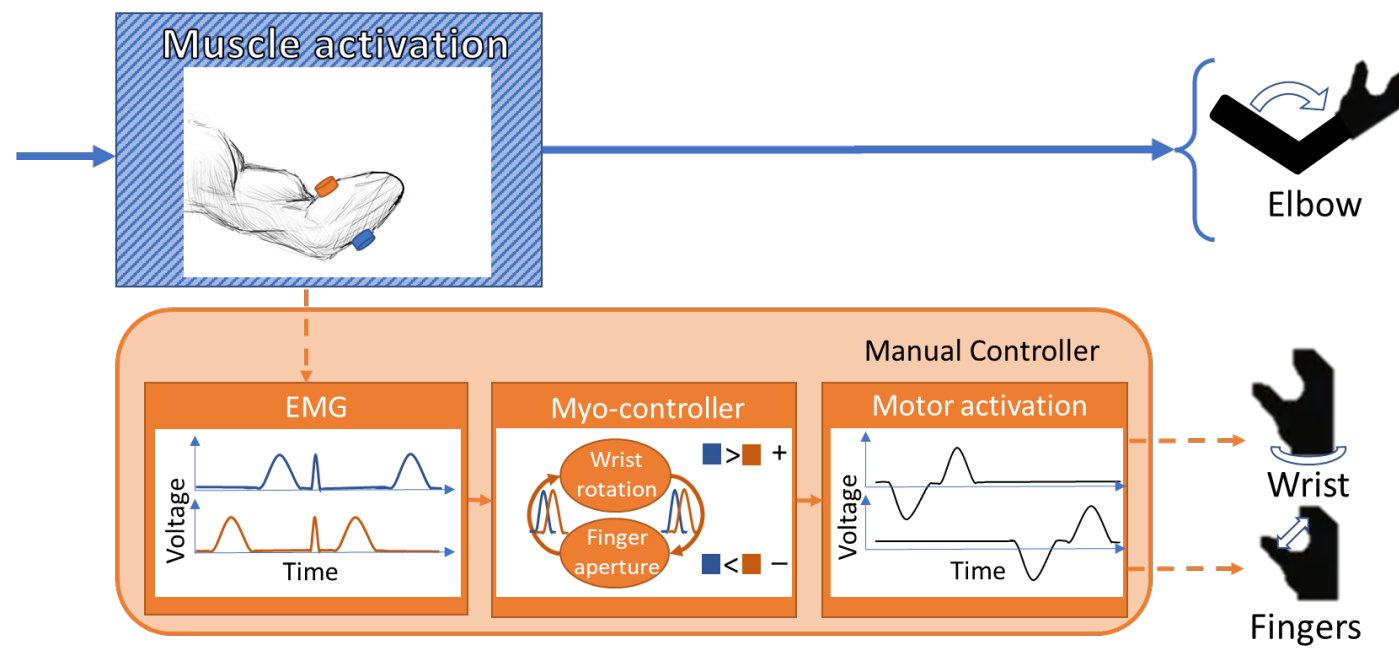

Figure 1.5 Illustration of the 2-channel control. In blue are the remaining functionalities handled by the user. In orange is the replacing solution to actuate the missing limbs. The 2-channel control can be decomposed into three phases: EMG acquisition, signal processing, and motor activation

Until recently, the control of myoelectric prostheses was nearly exclusively based on a similar paradigm implemented in the first myoelectric prostheses, namely, the "two-channels control". This implementation bases the control on two electrodes placed on two antagonists' muscles. These electrodes acquire the EMG signals produced by the muscular fibres when contracting. These signals are amplified and filtered to extract the signal's power, called "envelope of the signal", before sending it to the controller. The role of this controller is to map the pattern of EMG signals produced by the user's muscles to the desired prosthesis actuation. In the case of single DoF prostheses (often opening/closing), the controller has to determine three things: whether the prosthesis should move, in which direction the prosthesis should move, and at what speed, for prostheses allowing a regulated actuation. Hence, the prosthesis is actuated only if at least one of the envelopes exceeds the activation threshold. A 


\section{Chapter 1: Introduction}

clinician determines this threshold to avoid unwanted prosthesis activation due to movement or noise in the signal. When an envelope is high enough, the direction of actuation is determined by comparing the two envelopes. Each electrode is associated with a direction of the movement. The electrode placed on the flexion muscle (wrist or fingers) is often associated with the hand's closing and the other electrode in the opposite direction. When the flexion muscle electrode signal is higher than the other envelope, the controller sends a closing command to the prosthesis. Otherwise, the controller sends an opening command. The strength of the signal then determines the speed of the actuation. The prosthesis moves at minimal speed when the envelope just reaches the activation threshold and moves at the maximal speed when the envelope is slightly below the Maximal Voluntary Contraction (MVC) recorded by a clinician.

In the case of a multi-DoF prosthesis, the controller must, as a fourth task, select the DoF to be controlled. Hence, a state-machine is implemented. Each state corresponds to a DoF and behaves like the single DoF behaviour described above. The standard implementation requires the user to produce a cocontraction: a simultaneous, brief, and high contraction of the two muscles to switch from one state/DoF to another. To detect this cocontraction, the controller requires two windows of time without activations before and after a high-enough simultaneous activation of the two electrodes. The user needs to repeat this contraction pattern until reaching the desired DoF, with or without auditory feedback, to identify the new state of the controller at each change. The combination of the time window without activation and the time required by the cocontraction requires some time to select a specific DoF. This time required to select a DoF increases with the number of DoF proposed by the device and makes the control slow and unintuitive when controlling a multi-DoFs prosthesis [36]. 


\subsubsection{Pattern recognition-based myo-control}

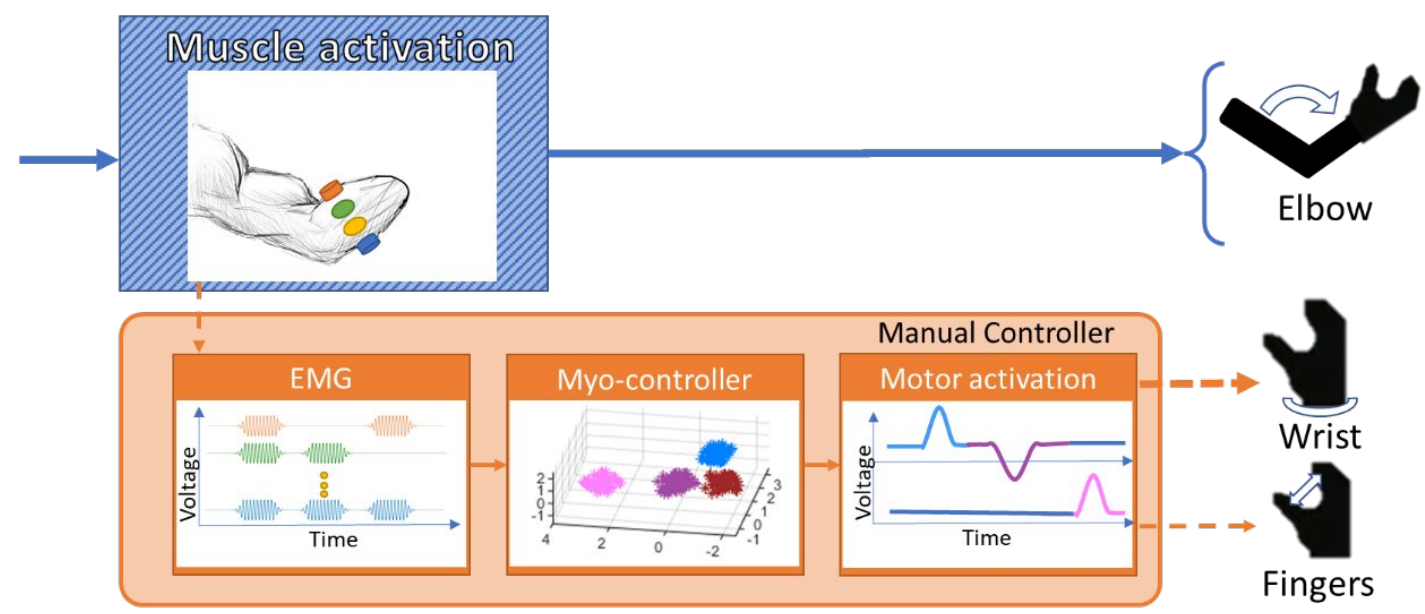

Figure 1.6 Scheme of a Pattern recognition based myo-control. A Raw of electrodes is placed on the limb. Each of them acquires EMG signals. All the channels are then processed to be classified into one pattern associated with one functionality of the prosthesis.

Another control type has been developed using more electrodes to acquire muscle pattern contractions to overcome this slow and complex DoF selection. Indeed, after an amputation or at the birth of limb-deficient children, some muscles controlling the different motions of the missing limb can remain. When attempting to perform specific movements with their missing limb, limb-deficient individuals contract the muscle or the set of muscles associated with this movement. Different movements are associated with different muscle activations and produce, therefore, different patterns of EMG signals. Pattern recognition based myo-control aims to recognise the pattern of muscle contraction associated with a specific movement and associate with it a specific prosthesis movement. This method requires a detailed acquisition of the EMG produced by the different muscle contractions. Therefore, most common implementations acquire the EMG signals through six to eight electrodes placed on the skin. For the same phantom movement, signals acquired by the electrodes may differ because of the noise caused by the skin, the cross-talk between muscles, and mainly because phantom movements are produced by a set of muscles potentially located differently for each limb-deficient individual. Therefore, pattern recognition myo-control requires machine learning (ML) algorithms to learn and adapt to each different anatomy and the current electrode state. 


\section{Chapter 1: Introduction}

ML algorithms are algorithms using data to determine the rules by themselves. The most common ML myo-controller implementations use supervised algorithms and require a training phase before using the device. During the training phase, the user must perform several specific movements of the hand and the wrist to contract his muscles, potentially performed at varying levels of contractions to increase the diversity of the data. Subsequently, the computer splits the EMG data into time windows with a recommended duration of $150 \mathrm{~ms}$ [37]. It computes the different descriptors for each of these time windows (Root Mean Square, Slope Sign Changes, Waveform Length, ...[38], [39]). The machine learning algorithms build the model based on these descriptors and the a-priory knowledge of the data.

Two categories ML algorithms are used to control upper limb prostheses. Classifiers associate the acquired pattern of muscle contraction (described by the descriptors) to the modelled movement producing the most similar EMG signals. At each time window, classifiers will associate the muscle contraction produced by the user to a single prosthesis functionality such as opening, closing, pronation, supination, etc. Examples of algorithms belonging to this category are algorithms such as linear discriminant analysis (LDA) [40], support vector machine [41], [42], or neural networks [43]-[45]. Compared to the two-channels myo-control, classifiers effectively remove the time required to switch between the different prosthesis' functions [46], and consequently, significantly improve the capability of the user to perform activities of daily living (ADLs). On the other hand, regressors decompose the acquired pattern of muscle contraction, here also described by the descriptors into a combination of modelled movements. Regressors thus enable the user to manipulate several DoFs of the prosthesis by combining the trained patterns such as opening while pronating, see for example [47], [48].

However, some drawbacks still limit their use. First of all, the current myocontrol only relies on surface EMG (sEMG) electrodes. These suffer from many factors lowering the accuracy, such as muscle fatigue, electrode shifts, arm posture, learning/adaptation of user [49]. These factors are the main reason for the lack of robustness restraining the practical application of machine learning algorithms [46]. Furthermore, Li et al.[50] studied the number of classes usable in practice and obtained a drop of accuracy when classifying more than six classes with amputee participants 


\section{Chapter 1: Introduction}

using six electrodes (the recommended number nowadays [39]). Additionally to the low average number of directly controllable DoFs, the generation of sufficiently distinct and reproducible muscles patterns is very variable between individuals [51], leaving some individuals unable to exploit the potential of their prosthesis. Finally, controllers based on machine learning algorithms are very sensitive to changes in the muscle patterns detected by the electrodes. They require, therefore, frequent recalibration of the contraction pattern model, which takes time for the user. These constraints on the usability of ML algorithm-controlled prostheses underline the importance of improving the HMI as it is currently a limiting component of the system.

\subsection{Technical solutions to improve manual control}

Several solutions have been developed to increase or compensate for pattern recognition algorithms' problematic accuracy and robustness. They aim to provide better quality signals through the intramuscular EMG acquisition, diversified sensors and use other channels to control the hand in parallel of the myocontrol.

\subsubsection{Intramuscular EMG acquisition}

Intramuscular electrodes are currently developed since most of the artefacts described above are caused by the distance between the electrodes and the muscles and by the skin separating them. Intramuscular EMGs can be obtained by either fixing an electrode in the muscle or the motor nerve addressing this muscle. Thus, the signal acquired is expected to be cleaner than signals obtained with sEMG electrodes. This signal can then be classified and enable fine control of the prosthesis [52]. Compared to surface electrodes, it showed better performance in terms of control quality and a better ability to maintain the desired muscle contraction pattern while showing similar performance in terms of throughput (tradeoff of speed and accuracy) and completion rate [53]. However, this method is still limited in practice. This technology requires a heavier medical procedure implying potential surgical risks, which is a big concern for the potential users [54]. It also requires a high power consumption and is sensitive to potential electrode drifts, which lower the quality of the signal and potentially 


\section{Chapter 1: Introduction}

require a new surgical intervention [55]. Furthermore, this method does not answer all the drawbacks of pattern recognition myo-control as it has been shown that this method still suffers from limb position effects [56]. For these reasons, the application of intramuscular EMGs is still limited to a minimal amount of individuals with limb deficiencies.

\subsubsection{Diversifying the sensor modalities}

Alternative modalities to EMGs are currently under investigation to provide sensors that, when placed in contact with the skin, could provide better information of the contraction of the muscles and be less influenced by the factors usually decreasing the accuracy of EMG electrodes. These sensors can then be combined or replace the traditional EMG interface. The techniques that associate data from diverse modalities are called sensor fusion techniques [57], [58]. Diversifying modalities can bring a context to interpret the standard EMG data better or acquire the same physical process while not being sensitive to the same noise as EMG signals and therefore complete them.

Bioimpedance [59], [60], near-infrared spectroscopy [61], and ultrasound have, for example, been used alone or in combination with EMG to improve the accuracy of the classification of muscle patterns[62], [63].

Another well-studied sensor combined with EMG sensors is the inertial motion unit (IMU). As the arm moves through space, the angle of the elbow and the effect of gravity change. These changes affect the position of the forearm muscles relative to each other and the skin. This effect, called the limb position effect, is one of the main causes of muscle contraction pattern misclassification. IMUs are sensors gathering accelerometers and gyroscopes providing orientation data. Fixed on the user's forearm, IMUs act as proprioception sensors by acquiring the forearm's position, orientation, and movement. This artificial proprioception could bring valuable information to reduce the limb position effect. The combination of acceleration data with EMG data can be done in several ways, as studied by Fougner and colleagues in 2011. The authors compared four different controllers. The first controller was a baseline made of a single classifier trained with EMG data on a single limb position representing the classical training. The second controller was a single classifier trained with EMG data 


\section{Chapter 1: Introduction}

on multiple limb positions representing an advanced training that would aim to reduce the limb position effect while still a pure EMG-based HMI. The third controller combined several classifiers trained with EMG data, each on a different limb position. A second classifier, trained with acceleration data, continuously estimated which EMG classifier has been trained at the closest limb position to the current one. Eventually, the fourth controller was a single multimodal classifier combining EMG and acceleration data on multiple limb positions. Results show that the best accuracy is obtained with the fourth controller when using a multimodal classifier. The combination of several EMG classifiers with the acceleration classifier comes at second place and the multi-position classifier at the third one [64]. The use of IMUs has been further researched 1) by using non-linear classifiers [65] to avoid the assumption that the limb position has a linear effect on the EMG signals and 2) by using the angular velocity and the magnetic field in addition to the acceleration [66].

These solutions aim to improve the accuracy of ML classifiers but are still sensitive to the temporal variability of EMG signals. They also require the user to control the whole prosthesis through his/her muscle contraction. Doing so, the user is still constraint by a limited number of controllable DoF, which he or she has to control sequentially.

\subsubsection{Using other modalities to control the prosthesis}

Rather than applying diverse sensors only to acquire and interpret muscle contraction patterns, other modalities can be used to directly control the prosthesis with myo-control. This addition of a new channel to the standard myo-control interface aims to enlarge the bottleneck of the control of the prosthesis. 


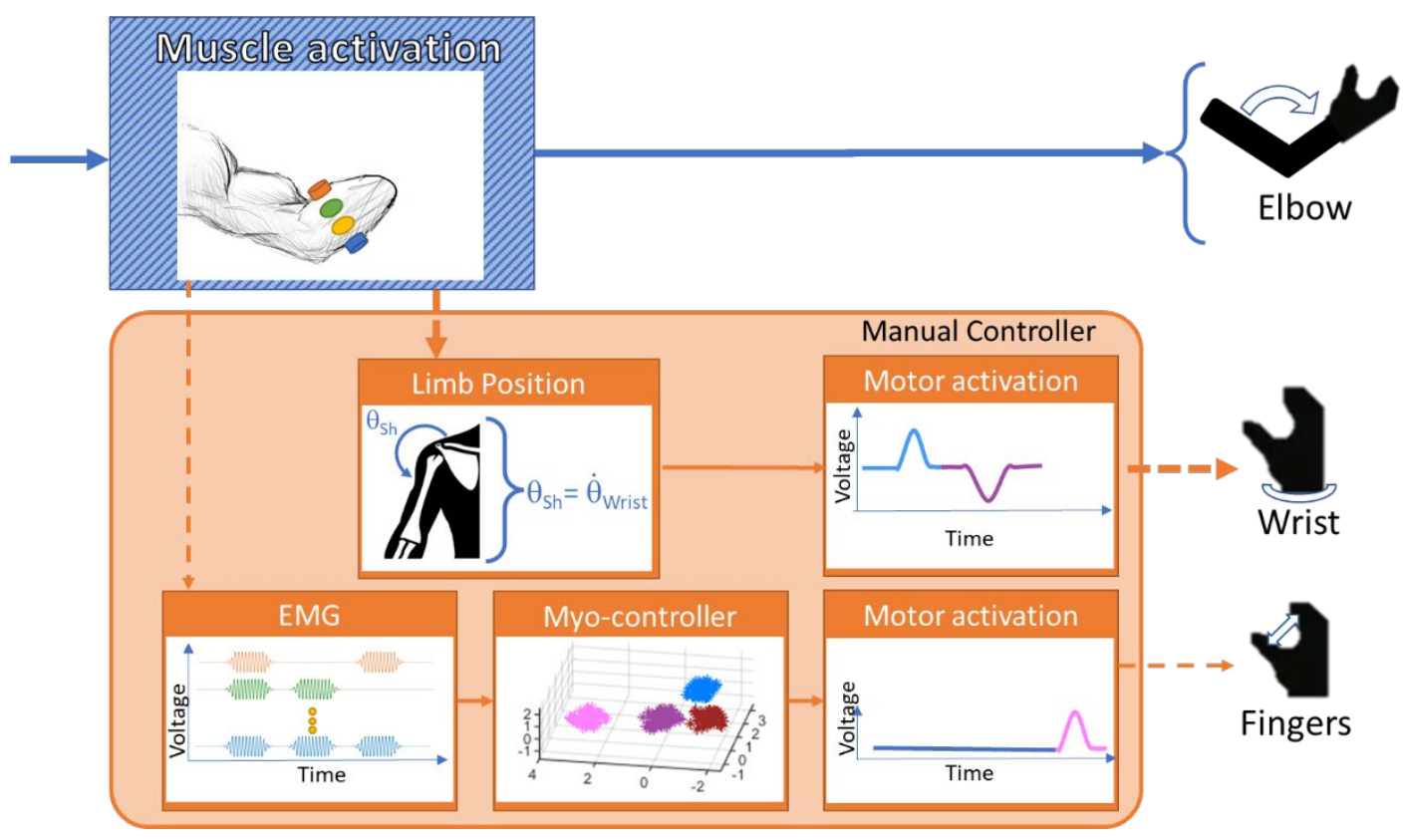

Figure 1.7 Illustration of the use of another modality to control one functionality of the prosthesis. Here the aperture and grasp type is control through a pattern recognition myocontrol, whereas the rotation of the wrist is controlled through the elevation of the shoulder

Investigating the use of the shoulder elevation to control the wrist rotation, Bennett and Goldfarb (2018) proposed a direct control of the speed and direction of the rotation of the wrist through the elevation of the shoulder [67]. This method splits the control of the different DoFs of the prosthesis over several modalities. The system has been compared to a classical two-channels control and has been shown to be $33 \%$ faster to complete the standard tests.

The tongue's position in the mouth has been used to select the grasp type to be used [68]. While intrusive, this system was faster than a traditional two-channel control since the user does have to rotate through the activation mode with Cocontraction. 


\section{Chapter 1: Introduction}

[69]. A movement of the forearm triggered the system. Then the user waited for the electrodes to stimulate the pattern associated with the grasp type he/she wanted to use and then reach the object. Once this reaching movement was detected through the accelerometers, the hand prosthesis was preshaped, and the user just had to close the prosthesis through 2-channels control. The performance obtained was similar to the two channels control alone but could be extended to propose the selection of more functionalities.

Arguing that vision has a preponderant role in the prehension process, Controzzi et al., in 2013, designed a system using the movement of the user's eyes to select the good grasp type [70]. The user had to follow the target object's shape with the eyes, while his gaze was followed through electrooculography. Assuming that the different forms of the objects would give the different patterns, the EoGs were then classified to select the grasp type to be used with the object. The study concluded with the feasibility of such a system without measuring the performance or the workload. Relatively close to other HMIs, which include autonomous controllers. (see section 1.5.1), this system proves that information about the targeted object can be obtained through "vision" and can increase the time performance of the grasp. However, this system still uses the user as the motor of the artificial exteroception and requires him/her to perform an additional step when grasping an object.

The different modalities exploited to control the prosthesis increases the diversity of the input from the user that can be used to control the device. While the potential benefits of this method need further investigation, the limitations of myocontrol are again only partially addressed. New modalities at the charge of the user induce a new cognitive load to activate muscles that are not on the path of the natural prehension process. Consequently, the user will need to learn and adapt to use the hand prosthesis and include new movements to the prehension process to control a few additional DoFs compared to standard myo-control. 


\subsection{The limitations of manual control}

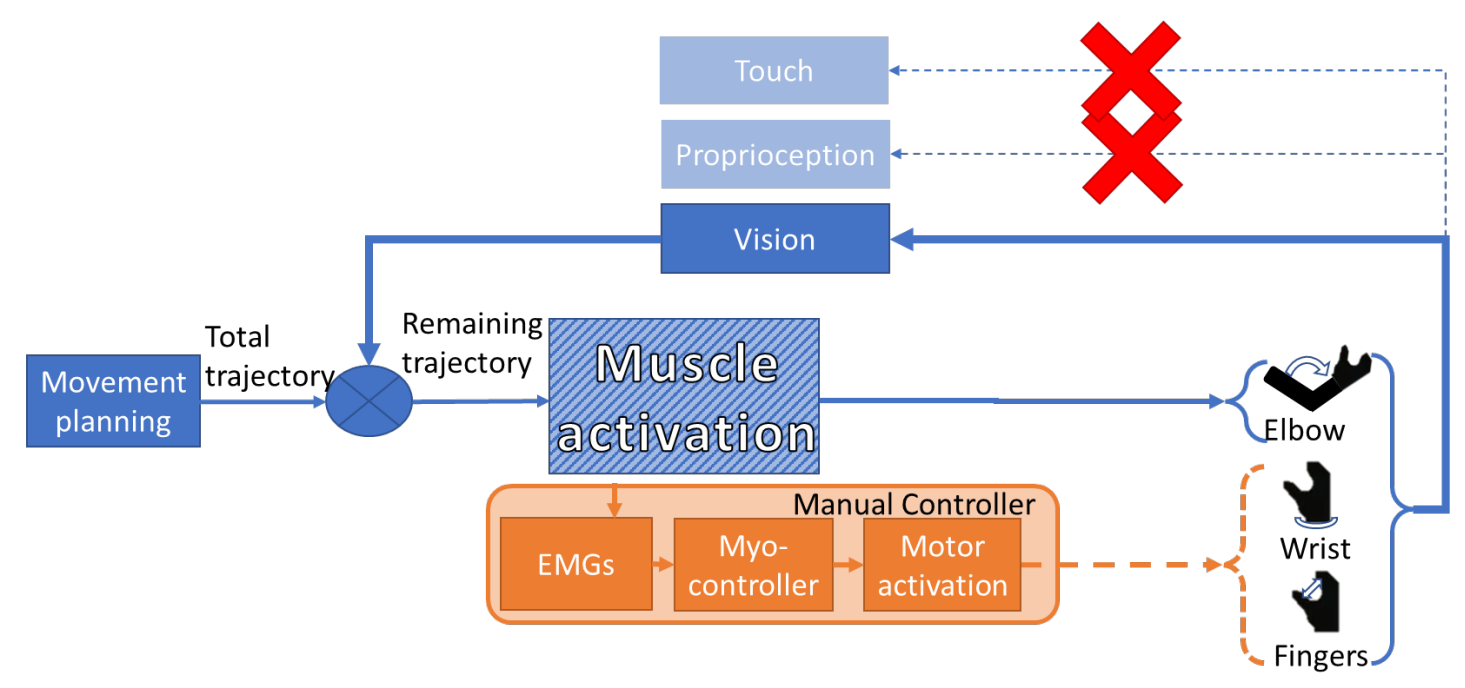

Figure 1.8 Illustration of the limitations of Manual control. Some functionalities usually handled by the individual are lost through amputation (light blue). This results in the increased used of the remaining sense to close the control loop. The modified anatomy of the arm also impacts muscle activation. The artificial path to actuate the prosthesis's functionalities relies on less stable and accurate signals.

The evolution of hand prostheses has provided them with a high number of joints, which has increased their dexterity. However, the control provided to the user has not followed this evolution. The myocontrol is limited to fewer functionalities than the healthy limb due to the state and the number of remaining muscles and the accuracy, resolution, and number of electrodes. The robustness of the myo-control is also impacted. The number of directly and simultaneously controllable DoFs, which is lower than the devices' capabilities, constitute a bottleneck in the human-machine interface due to the limited bandwidth of the myocontrol. This limited bandwidth prevents the user from benefiting from the latest mechanical development in hand prostheses.

The previously described technical solutions either achieve a more robust control by diversifying the sensors used for the muscle contraction classification, or increase the number of directly controllable DoFs. However, while definitively extending the capabilities of the myo-control, these techniques do not offer enough control modalities or classification outputs to control all the DoFs of the modern hand prostheses directly. For example, the relatively high number of different movements classified using ultrasound in [62] (15 movements including combinations of wrist 


\section{Chapter 1: Introduction}

and finger movements) is not high enough to offer direct control of the 20 grasps types proposed by the most dexterous prosthesis currently available nor the 33 human grasps types. This lack of direct control gets even larger when adding the control of the wrist to reduce the compensatory movements. Therefore, the user is still limited in the dexterity with which he or she can control the hand prosthesis.

In addition to the limited and unnatural control, prosthesis users have to adapt to a new limb with different motions or missing natural DoF (prosthetic wrists are rarely active in all the directions), replacing a fully functional hand. These mechanical differences are accompanied by the loss of proprioception and sense of touch, enabling the individual to embody and control the limb. The proprioception missing, the individual has to rely on memory and visual attention to know the current position of the prosthetic limb. This affects the visual behaviour of prosthetic users who look at their prosthetic hand much longer during reach and grasp action at the expense of lower visual attention given to the object to grasp and the preparation of the following action [71].

\subsection{The semi-autonomous control}

Modern devices significantly increased the level of autonomy with which they perform the task they are dedicated to. From the coffee machine preparing the beverage at a suitable temperature and potentially at a good time to the cars autonomously following the car in front, the diversification and the multiplication of the embedded sensors offered the capability of replacing the human agent for several subtasks. These sensors also increased the capabilities of the human itself, enabling, for example, delicate surgical operations. Insensitive to this trend, the standard manual control of upper limb prostheses still completely relies on the user for each movement without supporting or relieving obvious parts of the control. As a result, the manual control for upper-limb prostheses is still limited and fails to offer complete and intuitive control of modern prostheses. The automation of some control parts to support the user has already been implemented in other rehabilitation fields. The adaptation of some gait parameters according to the terrain was implemented for lower 


\section{Chapter 1: Introduction}

limb prostheses [72], and partly autonomous driving wheelchairs enable physically or mentally disabled persons to still move [73], [74]. The following section will provide an overview of autonomous controllers with their functioning.

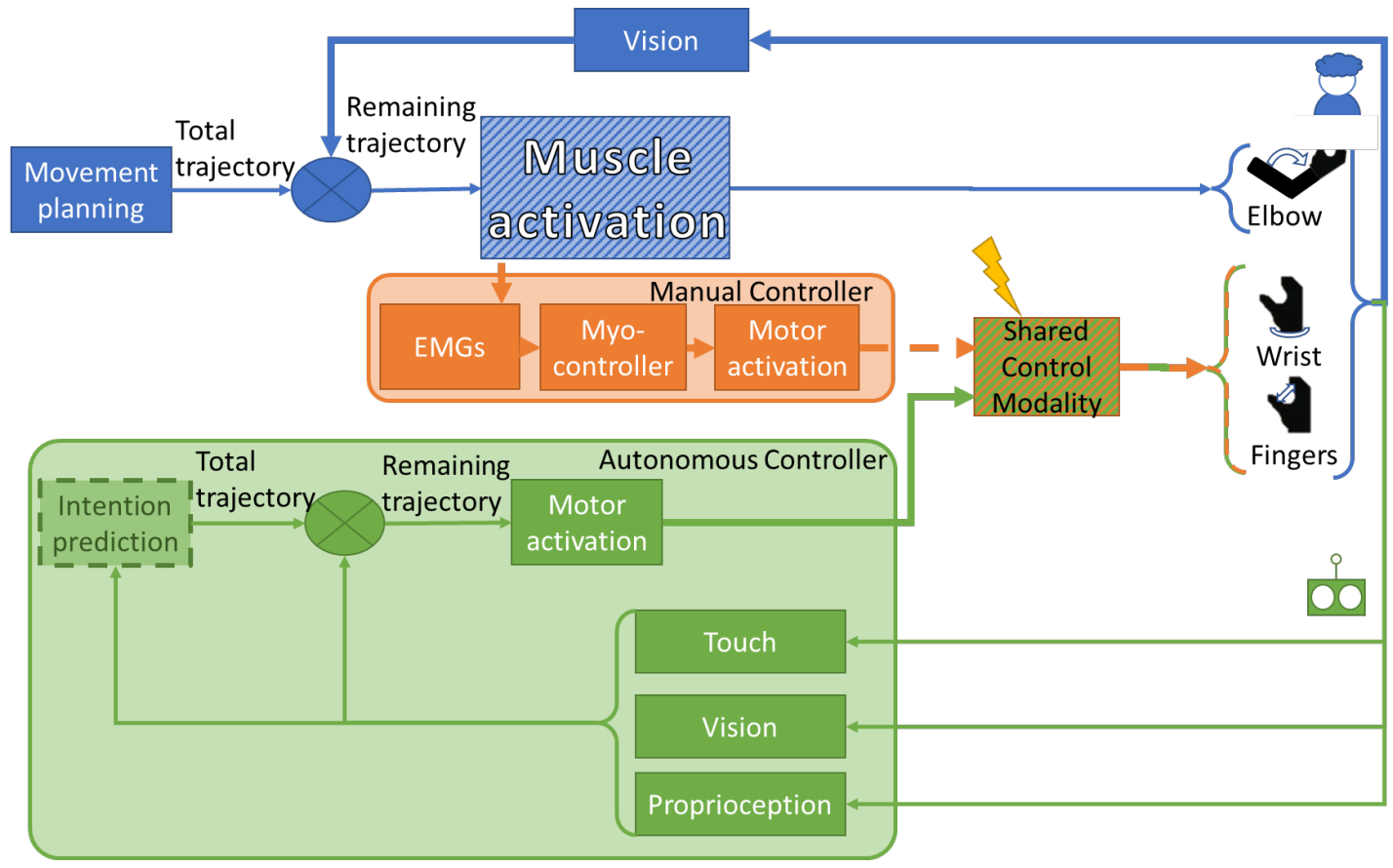

Figure 1.9 Scheme of functioning of a semi-autonomous system. The autonomous controller (green) reproduces the natural grasping process by gathering information through several modalities, emulating the natural touch, vision and proprioception, predicting the user's intent, and eventually preshaping the prosthesis using embedded sensors again. The commands from the user and the autonomous controller are combined according to the shared control modality.

Amputation or limb deficiency deprives the individual of the natural actuation channel and the feedback loop necessary for reasonable control. Standard control provides a replacement but with a limited number of inputs that disable the full recovery of capabilities. The goal of autonomous hand prosthesis controllers is to artificially reproduce the proprioception and/or the exteroception of the user to predict his/her intention and support the action by automating part of the necessary actuation. 


\section{Chapter 1: Introduction}

control is ruled by the shared controlled modality. The combination of the manual and the autonomous controller results in a semi-autonomous (SA) system where the action of the autonomous controller is conditioned by the user's decision and manual inputs.

The automation of the hand can handle all the DoFs of the device simultaneously without requiring direct supervision of the user. It can relieve the user of unnatural movement planning and adjust the actuation through artificial proprioception. It is also independent of the accuracy and robustness of the myocontrol and is therefore not affected when the accuracy of myocontrol is lowered. These qualities give autonomous controllers the potential to go beyond the current limitation of manual control, namely the limited number of directly controllable DoF and the associated cognitive workload.

\subsubsection{Intention prediction and associated automation}

The intention detection, the core of the autonomous controller, provides the goal to be achieved by the system. The following automation is a direct consequence of the amount of information predicted by the system. Several technologies have been studied, and their feasibility has been demonstrated. These diverse methods used to predict the user's intention provided either information on the object targeted by the user, on the specific grasping strategy intended by the user, or both, and at different time points of the reach-and-grasp action. This section will provide a quick overview of the solutions researched, which can be classified into prediction before the movement, during the grasp and the whole movement.

\section{I) Prediction before the movement}

Correctly preshaping all the joints of the prosthesis to give the user maximum support when grasping an object is a challenge. The more information the autonomous system has, the more accurate the predicted solution will be. For an autonomous system to provide a natural-looking movement and provide the best time performance when grasping an object, the time point of the prediction and, therefore, of the solution computation is fundamental, the earlier being the better. Therefore, the challenge is to gather all the information and predict the intention in the shortest time after the user's decision. Hence, several methods using computer vision have been proposed. 


\section{Chapter 1: Introduction}

Computer vision is the processing of data acquired by an optical sensor such as a camera providing colours (RGB) or colours and depth (RGB-D) to extract information from the context and the object of interest. The selection of the object of interest in the scene is the first issue the autonomous controller has to answer.

The first method to select the object before starting the movement is to give an active role to the user in selecting the object. Several systems proposed to do so by requiring the user to present the object to the camera. The object's selection in the camera's field of view is either done by selecting the closest object in the centre of the field of view [75], [76] or requires the object to be the only one in front of the camera [77], [78]. Thus, for the system to support the grasp of the right object, the user needs to pay attention to the camera's field of view according to where the camera is placed.

When the camera is placed in the hand prosthesis, such as in [32], [76], [77], [79], [80], the user is required to point to the object with the hand. Without feedback on the width of the field of view, it may be necessary to train the user to perform this movement [77]. When the camera is placed on the head, the user needs to orient the head to strictly face the object [75], [81]. This focus on the object requires visual attention and deprives the user of gaze flexibility, especially in a scene with many objects. Augmented reality has been used to help the user to select the correct object [75]. In both placements of the camera, the studies that tested the users required them to trigger or confirm the object selection, and the computed prosthesis preshape by a pattern of muscle contractions.

A second method to select the object without requiring an additional active step during the prehension process of an object is to predict the grasping intention from physiological information such as the gaze. Gaze tracking enables the system to gather data on the visual attention of the user. Once the camera captures the scene, objects are segmented and localised in the user's field of view. A fixed gaze on a specific object selects it. The trigger starting the preshape is then either the activation of the myocontrol [82], [83], the start of a reach movement [84], or a specific electroencephalogram pattern [85].

Once the object has been selected, the autonomous system can compute the pose of the prosthesis, providing more support to the user for grasping the object. Once 


\section{Chapter 1: Introduction}

the object has been selected, the autonomous system can compute the pose of the prosthesis, providing more support to the user for grasping the object. Humans grasp objects according to their shape and the experience they have with the object. It is, therefore, a combination of a-priory knowledge and situational information. The grasp of a milk carton can illustrate this. The shape is very regular and provides a good grasp from several sides. The grasping strategy may be different between 1) a situation where the individual wants a fast grasp to relocate the object, probably grasping the side of the carton, 2) a situation where the individual plans to put the carton in the refrigerator door, potentially grasping the top of the carton to avoid any disturbance in this crowded situation, or 3) a situation where the user wants to check the expiration date of the milk, grasping the side of the carton with a fully pronated forearm to anticipate the manipulation (end-state comfort [86], [87]). Three methods have been proposed in the literature: object recognition, object classification and object analysis.

\section{a) Object recognition}

A-priory knowledge can be provided to the system by associating the most used grasp to the object of interest. Therefore, the autonomous system uses computer vision to recognise the object by associating a correspondence from the training database. Once the match is found, the database provides the grasp strategy associated with the recognised object. The computing power and the size/resolution of the camera are two key factors impacting these algorithms' accuracy and robustness. Object recognition has been done in the upper limb prosthesis field with a lowresolution camera embedded into the hand [79] and a processor embedded into the hand [88]. The computation can also be distributed, as it has been done, on the user's smartphone [79] or a remote server [89].

The use of embedded hardware shows the technical feasibility of systems independent from computers. However, these studies only assessed the accuracy of the object recognition in this particular condition. The integration of this automatisation in an HMI and assessing performance gain had not been done yet. 


\section{Chapter 1: Introduction}

The object recognition method comes with two difficulties. The first one is that any object not belonging to the training dataset is not recognised or wrongly associated and therefore does not carry any a-priory information. The size of the dataset is therefore critical. Furthermore, any new entry in the dataset requires the algorithm to be retrained, which consumes and requires consequent computational power. The second difficulty is the fixed grasp strategy per object, which does not consider the context, such as surrounding obstacles or the manipulation planned by the user.

\section{b) Object classification}

Algorithms of computer vision made some progress in understanding and classifying objects. Instead of recognising an object previously trained as the methods described previously, several studies proposed directly classifying the object of interest to associate an appropriate grasp strategy. This method enables to preshape the hand when facing an object that does not belong to the training set. Using an RGB camera placed on the table to classify objects into four grasp strategies obtained $88 \%$ success rate classification when assessed with prosthesis users [78]. Unfortunately, the gain of performance when using the system has not been assessed. Using an RGB-D camera, a latter placed on the table implemented a neural network to classify the object into four grasp strategies. Compared to the previous one, the object classification accuracy improved for the object belonging to the training dataset and classified with $93.9 \%$ accuracy objects not belonging to the training dataset, demonstrating the flexibility of the method.

Object classification answers one of the weaknesses of the object recognition method by not being restrained to the training dataset, which potentially enables a more extensive diversity of correctly handled objects. However, it shares the single grasp strategy per object with the object recognition method, including the user's exact intention. 


\section{Chapter 1: Introduction}

\section{c) Object analysis}

Focusing on the context, such as the shape of the object and its current orientation, several methods are proposed to analyse the target object right after its selection to compute the prosthesis' pose required to grasp the object.

The first implementation combined an RGB camera fixed on the wrist with a laser sensor acquiring the distance to the object [80]. The object's size in the camera's field of view was thus combined with its distance to obtain the width of the object's side facing the camera and consequently the hand. Based on this width, the system preshaped the fingers and the wrist of the prosthesis.

Placing the cameras on the user's head, a second implementation used stereo vision to acquire the shape of the object and its orientation [75]. The thinnest side of the object was prioritised, and the prosthesis was preshaped, consequently adjusting the grasp type, the aperture of the fingers, and the hand's rotation when triggered by the user. This method to determine the grasp type and the aperture of the fingers has been assessed against the standard two-channel control and demonstrated better time performance [81]. The experimental setup was limited to a reach-and-grasp task with one single object on the table.

The weakness of these methods is that no a-priory knowledge is provided on the object, meaning that the cup's body and handle will be considered in the same way.

Eventually, a solution replacing computer vision with oculometry was explored by Controzzi et al. in 2013 [70]. The user was required to follow the object's shape with their gaze, producing electrooculographic signals classified into prosthesis preshape. The visual attention required to use the system places the individual at the core of the intention prediction. 


\section{Chapter 1: Introduction}

\section{II) Automation during the grasp}

One of the primary use of myoelectric prostheses is to hold objects or to support the manipulation [90]. While better control of the wrist and the fingers during the reaching phase may make the affected side more active, supporting the grasping phase already impacts the current use of the prosthesis. Support can be brought automatically through tactile sensors embedded in the prosthesis to gather data about the object and its situation.

Relatively early, tactile sensors embedded in the prosthesis have been used to detect a slip of the object [91]. After detection, several strategies have been developed to counter the object's fall, such as increasing the force exerted by the prosthesis' fingers [92], [93]. The force increased the fingers' friction on the object and therefore stopped it from falling. However, it also led to a deformation of the object. Another method to stop the object's fall consists of automatically rotating the wrist of the prosthesis to better counter gravity [94].

Eventually, a method using tactile sensors embedded in the prosthesis enables determining the best finger configuration to fit the object's shape [95]. A deep-learning algorithm determines the adjustment of each finger to grasp the object optimally. This method includes a myocontrol with a high number of possible classes provided by high-density electrodes to condition the application of the computed solution to the similarity of this solution with the manual command. In discordant control, the myocontrol commands have priority, and the autonomous solution is not applied. This method has not been assessed with a real prosthesis but in virtual reality. Such prosthesis exists as a prototype [96]. In contrast with the previous methods, this method focuses on the very end of the reach-and-grasp movement by adjusting the grasp. Therefore, it does not determine the targeted object nor the rotation of the wrist.

\section{III) Prediction during the whole movement}

In the goal of relieving the user from any active input to predict the object and the grasp strategy, information from the reaching movement has been included by several studies. 


\section{Chapter 1: Introduction}

A first solution studied the feasibility of combining the information from the gaze in the scene and the information from the forearm's movement [97]. Hence, the subject's gaze was tracked, and two cameras were fixed on the head and wrist. Crossing the head-mounted camera with gaze tracking enables one to determine an area of interest. Combined with the movement of the images acquired by the wristmounted camera, it was possible to determine both the type of movement of the hand (reach, rotary, linear, and retreat) and the object of interest of the subject while taking into account the discrete behaviour of the gaze fixation.

It is also possible to combine gaze tracking information with EMG data from the arm to estimate the final position of the hand [84]. Associated with information on the surrounding objects, it would enable selecting the object and determining the side of the object that the user wants to grasp. The method proposed predicts the position first through gaze information then updates the prediction through EMG data to follow the movement of the arm. This enables to take the gaze very early into account and relieve the user from forced visual attention later in the movement.

The possibility of completely removing the gaze tracking and using only the camera fixed on the wrist to predict the object of interest has also been demonstrated [98]. The authors propose to analyse the objects when the hand is far enough from the table to have all the objects of the scene present in the image. Then the movement of the image during the reaching movement would select the object through a deeplearning algorithm. Similar to the previous methods exposed, the prediction accuracy increases during the reaching movement to reach a maximum at the object's vicinity. The timing to preshape the prosthesis in the flight would need to be attentively studied to combine an actuation of the prosthesis early enough to be in position at the end of the reaching movement while still ensuring a stable goal. It should be noted that the studies previously cited did not assess the performance during clinical trials.

On the other hand, several studies proposed to predict the wrist's rotation only based on the orientation of the user's arms, acquired through inertial motion units (IMUs). Synergy was first reported between the wrist rotation and the shoulder adduction/abduction during natural reach-and-grasp actions [99]. The authors proposed to use this synergy to automatise the wrist rotation to improve the fluency 


\section{Chapter 1: Introduction}

and skill of amputees but did not present a concrete implementation. This synergy has been used in two studies.

The first study proposed to detect and reduce compensatory movement of the shoulder elevation[81]. Indeed, limb-deficient individuals rely more on the shoulder joint to compensate for their lack of forearm pronation/supination [100]. The system monitored the orientation of the upper arm from the affected side and answered to a high shoulder abduction by rotating the wrist to complete the movement. This method was assessed through a clinical trial and demonstrated a gain of time performance compared to the standard two-channels control.

The second study used the rotation of the unaffected side and the relative movement of the two hands to determine the current action during the bi-manual task [101]. Actions were then classified as idle, unilateral if the affected side is the only one moving, bi-manual asynchronous if the two hands move toward each other, and bi-manual synchronous if the two hands move in the same direction. This enabled the system to assist the user by automating the rotation of the wrist of the prosthesis. During unilateral actions, the wrist's rotation could be controlled through the shoulder abduction/adduction. During bi-manual asynchronous actions, the controller rotated the wrist so that the two palms faced each other. Eventually, during bi-manual synchronous actions, the controller mirrored the rotation of the sound forearm with the prosthetic wrist. This method also showed a gain of time performance compared to a standard 2-channel control.

\subsubsection{The rules governing the semi-autonomous system operation}

The rules for distributing the control of the same DoFs of the device to two different agents are critical for the task's performance and success rate. This is why collaborative control is a very studied field in robotics and rehabilitation [74], [102], [103]. The distinction is made between traded control, where the user and the autonomous controller have the control sequentially but never simultaneously, and shared control, where the two agents can control the device simultaneously. The distinction is also made between the different agents' roles, such as supervision when 


\section{Chapter 1: Introduction}

planning the movement and operating the device's actuation [104]. In some of the systems previously presented, both the user and the autonomous system had both roles. The user targeted the object and potentially planned the reach-and-grasp movement required (supervision), but also moved the forearm and controlled the prosthesis through myocontrol to adjust or close the fingers (operation). The autonomous system automatised the preshape of the hand by actuating some of the DoF of the hand (operation), but also determined alone which grasp strategy to apply to the target object (supervision).

This collaborative aspect has not been studied explicitly in upper limb prosthesis implementations. Several designs have been implemented without being compared, and their specific impact on the qualitative and quantitative outcomes is still unknown. Some designs implemented a traded control where the user had to trigger the punctual support of the autonomous system [75], [78], some others implemented a traded control where the autonomous system operates the prosthesis as long as the user does not control the prosthesis [101]. Shared control has been proposed through the conditioning of the application of the computed solution to similar myo-control commands [95]. The autonomous controller completes the actuation intended and partially actuated by the user. Another shared control method implements the prediction of the target position of the prosthesis during the actuation of one of the DoFs. The other DoF is then adjusted to reach the predicted position following the manually controlled joint [105].

\subsubsection{Current limitations of semi-autonomous systems}

The human grasp has been frequently studied in an attempt to describe and predict it. Given a specific object, a study showed that $96 \%$ of the subjects tended to grasp it by its thinner side [106]. This same study shows that the size of the object and its weight influence the choice of grasp type to grasp it. Another study involving manipulations showed that the "precision prismatic" and "power prismatic" grasps are more likely to be used when grasping an object [107]. These results encouraged the development of autonomous controllers associating one grasp type per object. However, these two studies also show no strict correlation between the object and the grasp type used. Indeed, the grasp type used for a specific object is also subject 


\section{Chapter 1: Introduction}

dependant [106] and task dependant [87]. This leads to the fact that associating a grasp type per object can lead to discrepancies between the user's intention and the prosthesis' automation. It can also require an adaptation of the user to the automation. In order to predict the grasping strategy intended by the user, more information has to be observed. Only a few studies, such as [81], [101], proposed such grasping strategy predictions, but they limited the prediction to the rotation of the wrist.

The methods based on computer vision can enable the actuation of the entire hand prosthesis, offering the preshape of both the grasp type and the wrist, bringing the prosthesis control at the level of its mechanical advancement. However, their application has been poorly tested. The protocols assessing the SA systems' benefits have been indeed limited to sparsely distributed objects on a table [75], [77], [85], [108], with the manipulation of a single object per trial.

Despite the numerous studies developing autonomous systems, the benefit of each of these systems in a clinical application context has been very little investigated. As cited earlier, several studies [77], [78], [88], [108] have highlighted the feasibility of designs of autonomous controllers but did not assess the interaction with the user within a SA system or the impact on the performance compared to myo-control. For the studies that compared the performance against myo-control, the comparison has been made against the standard commercial two-channel control [83], [103], nowadays outdated by machine learning algorithms already used in commercial systems. The increase of time performance and the decrease of physical and cognitive efforts due to using a SA system are still unknown.

ML-based controllers have been developed to enable the user to control several DoFs directly and it has already improved the intuitiveness and the time performance compared to the historical myo-control. The expected benefits of SA controllers also target the prosthesis's ease of use and time performance. The studies so far have shown that the SA is better than the two-channel [81], [101], but it is still unknown if the improvements brought by a SA system remain significant when combined with a more advanced myocontrol such as a muscle contraction pattern recognition. This lack of investigation prevents from justifying the development of SA systems over a solution, the advanced ML-myocontrol, which combines flexibility and proved benefits. 


\section{Chapter 1: Introduction}

Finally, SA systems induce a plurality of agents controlling the prosthesis. In these systems, two agents control the actuation of the prosthesis: the autonomous controller and the user. The shared control modality between the two agents has not been studied explicitly in the upper limb prosthesis field, where the user controls the entire forearm's movements and participates in the actuation of the prosthesis.

\subsection{Goal of this thesis}

The aim of this dissertation was to study how an intelligent controller that predicts and autonomously reacts to the user's intentions affects the user's performance and workload when it is combined with the state-of-the-art machine learning myocontrol (pattern recognition) and used to control an active 4-DoF prosthesis during the reach-to-grasp manipulation tasks. More specifically, this thesis has developed a method extending semi-autonomous systems' capabilities using computer vision and several sensor modalities. The improvement of the performance and the muscle usage of the combination of an autonomous controller with a pattern recognition myo-controller has been assessed. Eventually, a focus has been given on the shared control modalities' impact on the performances.

The rest of this dissertation is structured as follows:

- In chapter 2, a method answering some current limitations of autonomous controllers is developed. The design of a novel autonomous controller for 4DoF hand prosthesis (2-DoF in wrist and 2-DoF in hand) is exposed, implementing both artificial proprioception, exteroception, and short-term memory.

- In chapter 3, the potential improvement of the performance when associating a pattern recognition myo-controller with the autonomous controller developed in the previous chapter is examined. To this goal, the performances of able-bodied and amputee participants were recorded during clinical tasks, and several outcome measurements were analysed to provide a better 


\section{Chapter 1: Introduction}

understanding of how the novel autonomous system impacts the baseline performance of the myocontrol.

- In the light of the knowledge on the interactions between the user and the system acquired in the previous chapters, the rules that coordinate the autonomous and the manual controls were considered to impact the performance. Therefore, chapter 4 focuses on the impact on the performance of the shared control design when the goals of the two control agents differ. Hence, an autonomous controller and a pattern recognition controller were reproduced in a controlled environment. The measures of ten able-bodied participants using different shared control modalities under different levels of discrepancies were recorded and analysed. 
Chapter 2: Automatization of the wrist and the hand based on the prediction of the intended object's grasped side

\section{Automatization of the wrist and the hand based on the prediction of the intended object's grasped side}

As exposed in the introduction, the know benefits of the semi-autonomous systems to control an upper limb prosthesis are limited for several factors. First, the benefits of semi-autonomous systems are only known in the frame of the historical two-channel control [81], [101]. Second, autonomous controllers developed until now predict the object of interest and propose a single solution, ignoring the grasping strategy intended by the user. Lastly, while SA systems can potentially offer the control of all the available DoFs if the prosthesis and therefore solve the limited bandwidth of manual control, existing systems only actuate the wrist rotation when the wrist is handled.

From the existing technologies used to develop autonomous controllers, only those based on computer vision offer the potential of preshaping every DoFs of the wrist and the fingers to adapt to the grasp intended by the user. Existing systems based on pressure sensors need contact with the object to support the grasp and therefore 
Chapter 2: Automatization of the wrist and the hand based on the prediction of the intended object's grasped side

cannot assist the preshaping of the wrist. Existing systems based on the movement analysis through IMUs only assist one DoF of the prosthetic wrist. However, the existing systems based on computer vision have not been assessed in challenging environments such as 1) in a cluttered scene where several objects are present and in contact with each other, 2) dynamic scenes where objects are moved during the trial, or 3 ) standard tests. Therefore, the benefit of SA systems based on computer vision in terms of time performance and physical effort compared to SoA ML algorithms is not known in those specific conditions.

The method presented in this section aims to achieve the preshape of all the DoFs of the prosthesis by proposing several grasping types and including a two-DoFs active wrist based on the user's intention to support a reach-and-grasp task. This intention, combining object selection and grasp strategy, is therefore predicted by the position of the prosthesis placed by the user during a natural reaching movement. The preshaping support is designed to adjust the hand in real-time in the object vicinity and relieve the user from the necessity of an extra command or action to trigger the autonomous controller. Furthermore, the method presented aims to offer support to the user in complex scenes reproducing situations from everyday life comprising moving objects and crowded scenes.

\subsection{Architecture}

The system developed aims to support the user by predicting the intended grasp strategy and the target object. The proposed method combines the position of the hand and scene modelling to select the object of interest among the present ones and propose a grasp strategy enabling its grasp in the current position of the hand. Therefore, this solution requires acquiring the scene and modelling the different graspable objects present, which reproduces part of the human exteroception and tracks the movement of the prosthesis, which reproduces part of the human proprioception. The context, including scene and prosthesis, needs to be gathered to determine the user's intention and compute the consequent preshape. 
Chapter 2: Automatization of the wrist and the hand based on the prediction of the intended object's grasped side

The method proposed can be decomposed into several modules in charge of the different tasks just described. The artificial proprioception module tracks the position of the forearm, combining computer vision and IMU. The artificial exteroception of the module processes the scene in front of the user to obtain the position, shape, and size of the graspable objects through computer vision. The scene generating module combines the output of the two previous modules to reconstruct the situation in a game engine (Unity) and filter them, thus offering the ground for the user estimation module to predict the user's intention. Eventually, the preshape calculation module determines the placement of the prosthesis to grasp the previously selected object. In addition, the AR feedback module displays the processed scene, status and the autonomous controller's current decision through an augmented reality head-mounted display.

The implemented automation aims to actuate every DoF from the left-handed Michelangelo prosthesis (Ottobock, Duderstadt, Germany). This one has an active wrist with two DoFs (flexion/extension and pronation/supination) and a rotating thumb allowing two different grasp types (palmar and lateral). 
Chapter 2: Automatization of the wrist and the hand based on the prediction of the intended object's grasped side

\subsubsection{Artificial proprioception}

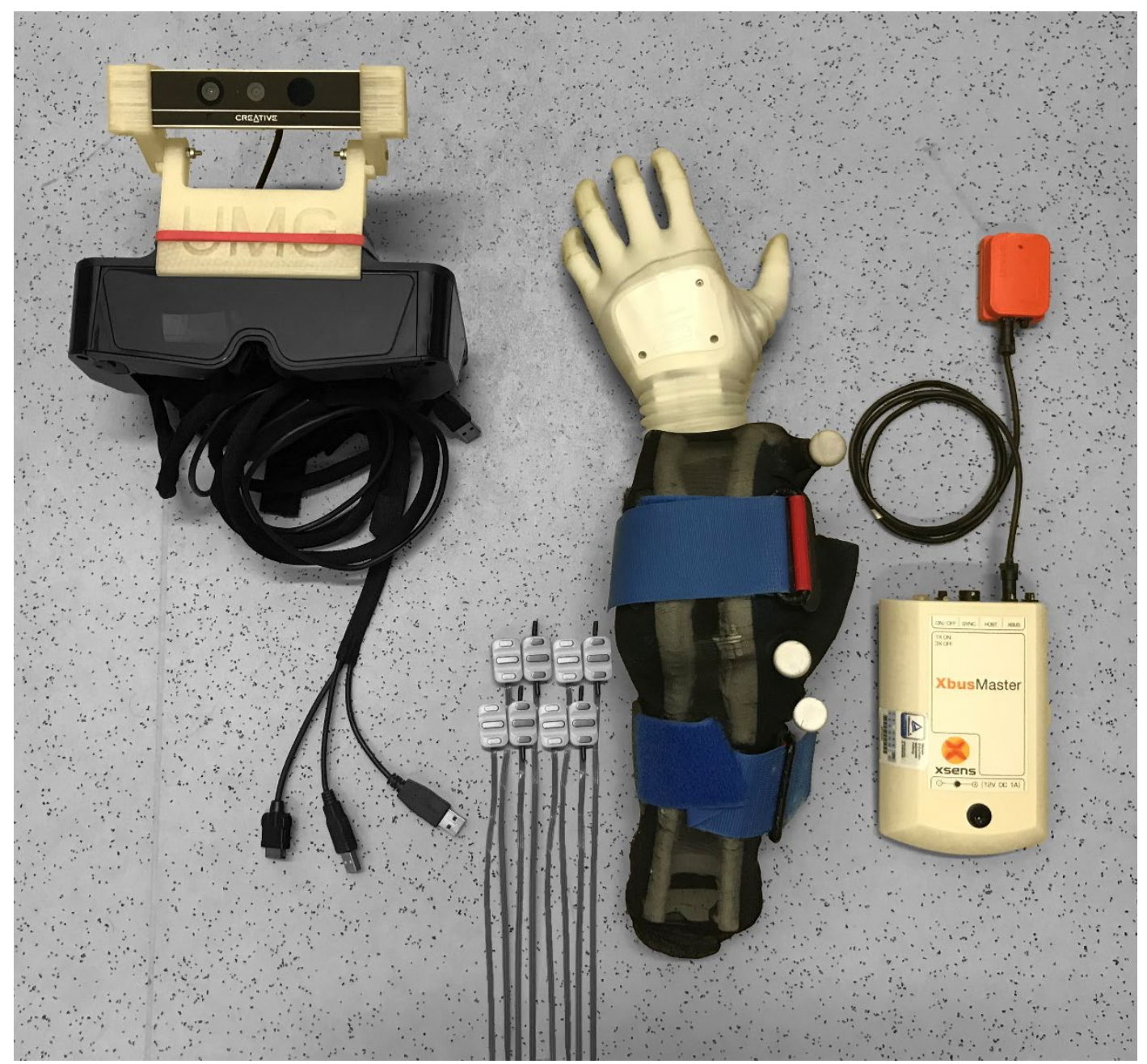

Figure 2.1 Photography of the material used with highlight of the components used for the artificial proprioception module. Those were the RGB-D camera, the sensors embedded in the prosthesis, the IR Markers placed on the socket, and the IMU fixed on the socket.

The artificial proprioception module first fixed the referential for the other sensors. Provided by the RealSense SDK, the camera's position was continuously given as its displacement compared to the first frame taken by the camera, a Creative SR300 (Intel Realsense, Santa Clara, California) illustrated in Figure 2.1. This displacement was translated into the camera's position by fixing the first frame as the origin of the generated scene.

To acquire the prosthesis position, the system used the three infrared markers fixed to the prosthesis. Since these markers are highly reflective for infrared light, a threshold was applied on the infrared data stream to detect highly reflective points in 
Chapter 2: Automatization of the wrist and the hand based on the prediction of the intended object's grasped side

the infrared stream of the camera (the threshold was fixed at $40 \%$ of the maximum detectable intensity). The binary picture (represented as black and white) was then filtered through a simple blob detection from the OpenCV library to detect the scene's round and highly reflective object. A set of filters were applied to remove noisy reflections:

- the area of the reflection had to be between 4 and 1000 pixels,

$$
\text { Circularity }=\frac{4 \pi \text { Area }}{(\text { perimeter })^{2}}
$$

- the circularity had to be higher than 0.15, with Area the area of the blob (round reflection) and perimeter its perimeter

$$
\text { Inertia }=\frac{\text { Side }_{\text {shortest }}}{\text { Side }_{\text {Longest }}}
$$

- the inertia ration had to be higher than 0.5 , with Side $e_{\text {shortest }}$ the length of the shortest side of the blob and Side $e_{\text {longest }}$ its longest side

$$
\text { Convexity }=\frac{\text { Area }}{\text { Area } a_{\text {convexHull }}}
$$

- the convexity had to be higher than 0.5, with Area $a_{\text {convexHull }}$ the area of the smallest convex shape containing the blob.

The $2 \mathrm{D}$ position of the detected blobs was then translated into a $3 \mathrm{D}$ position thanks to the depth data. The new reflections' position was then associated with the markers from the previous frame through the Hungarian algorithm [109]. This algorithm takes the previous positions of the markers and the new detected points and proposes pairs of new detection/old markers that minimise the distances between the positions of each iteration.

The average position of the three markers was then taken as the position of the prosthesis. Since edges of different lengths formed the triangle, the orientation of the retroreflective markers constellation in the camera's coordinate system has a single 
Chapter 2: Automatization of the wrist and the hand based on the prediction of the intended object's grasped side

solution based on the position of the three corners. This orientation was later used to compute the relationship between the coordinate systems of the IMU of the generated scene.

The prosthesis orientation was driven by the IMU (MTw Awinda, XSens, Enschede, Netherland), fixed on the sockets. The IMU was calibrated independently from the system at each new start to set its internal reference based on the gravity vector and magnetic north. The orientation of the forearm thus transmitted to Unity was related to this internal world coordinate system. The relationship between this internal world coordinate system and the coordinate system of the generated scene was required to include the forearm orientation into the generated scene.

When the retroreflective markers' constellation was entirely observed in the camera, the forearm orientation could be acquired from two sources: the orientation of the constellation in the camera's coordinate system and the orientation of the IMU in its own internal world coordinate system. The following equations give the relation between those two, given:

- $R_{\text {Prosthesis }}$, the rotation of the prosthesis in the coordinate system of the generated scene,

- $\quad R_{\text {Camera }}$, the rotation of the camera in the coordinate system of the generated scene provided by the Ego-motion mentioned earlier,

- $R_{\text {constellation }}$, the rotation of the constellation in the coordinate system of the camera provided by the processing of the infrared stream of the camera described earlier,

- $R_{\text {Const2Prosth }}$, the rotation of the prosthesis in the coordinate system of the marker's constellation measured on the prosthesis,

- $R_{\text {Reference }}$ IMU , the rotation of the reference of the IMU in the coordinate system of the generated scene, which we want to know,

- $R_{I M U}$, the rotation of the IMU in its referential, transmitted by the XSens software,

- $\quad R_{I M U 2 P r o s t h}$, the rotation of the Prosthesis in the coordinate system of the IMU measured on the prosthesis. 
Chapter 2: Automatization of the wrist and the hand based on the prediction of the intended object's grasped side

$$
\begin{gathered}
R_{\text {Prosthesis }}=R_{\text {Camera }} * R_{\text {Constellation }} * R_{\text {Const2Prosth }} \\
R_{\text {Prosthesis }}=R_{\text {Reference }_{I M U}} * R_{I M U} * R_{\text {IMU } 2 \text { Prosth }}
\end{gathered}
$$

The combination of the equations (2-4) and (2-5) leads to the following equation (2-6) used to calibrate the system:

$$
\begin{aligned}
R_{\text {Reference }_{I M U}} & =R_{\text {Camera }} * R_{\text {Constellation }} * R_{\text {Const } 2 \text { Prosth }} \\
& * R_{I M U}^{-1} * R_{I M U 2 \text { Prosth }}^{-1}
\end{aligned}
$$

Based on the IR markers constellation's centre and the forearm's orientation, the prosthesis model was placed and moved in the virtual space, providing the position of each component at any time for the prediction of the user's intention.

\subsubsection{Artificial exteroception}

The artificial exteroception module aimed to exploit the depth stream of the RGB-D camera to extract the graspable objects standing in the camera's field of view from the scene. Frames were extracted at a frame rate of around two Hz. In order to avoid noise caused by an image moving too fast, the image acquisition was conditioned by a low translation speed (below $10 \mathrm{~cm} \cdot \mathrm{s}^{-1}$ ) and rotation speed (below 30 deg. $\mathrm{s}^{-1}$ ) of the camera fixed on the head. The point cloud processing was split into two sequential threads and used the point cloud library (PCL) [110]. PCL is an open-source library focusing on processing point cloud data and comprising state-of-the-art methods to segment and model the data. These threads could be executed in parallel to double the frame rate. 
Chapter 2: Automatization of the wrist and the hand based on the prediction of the intended object's grasped side
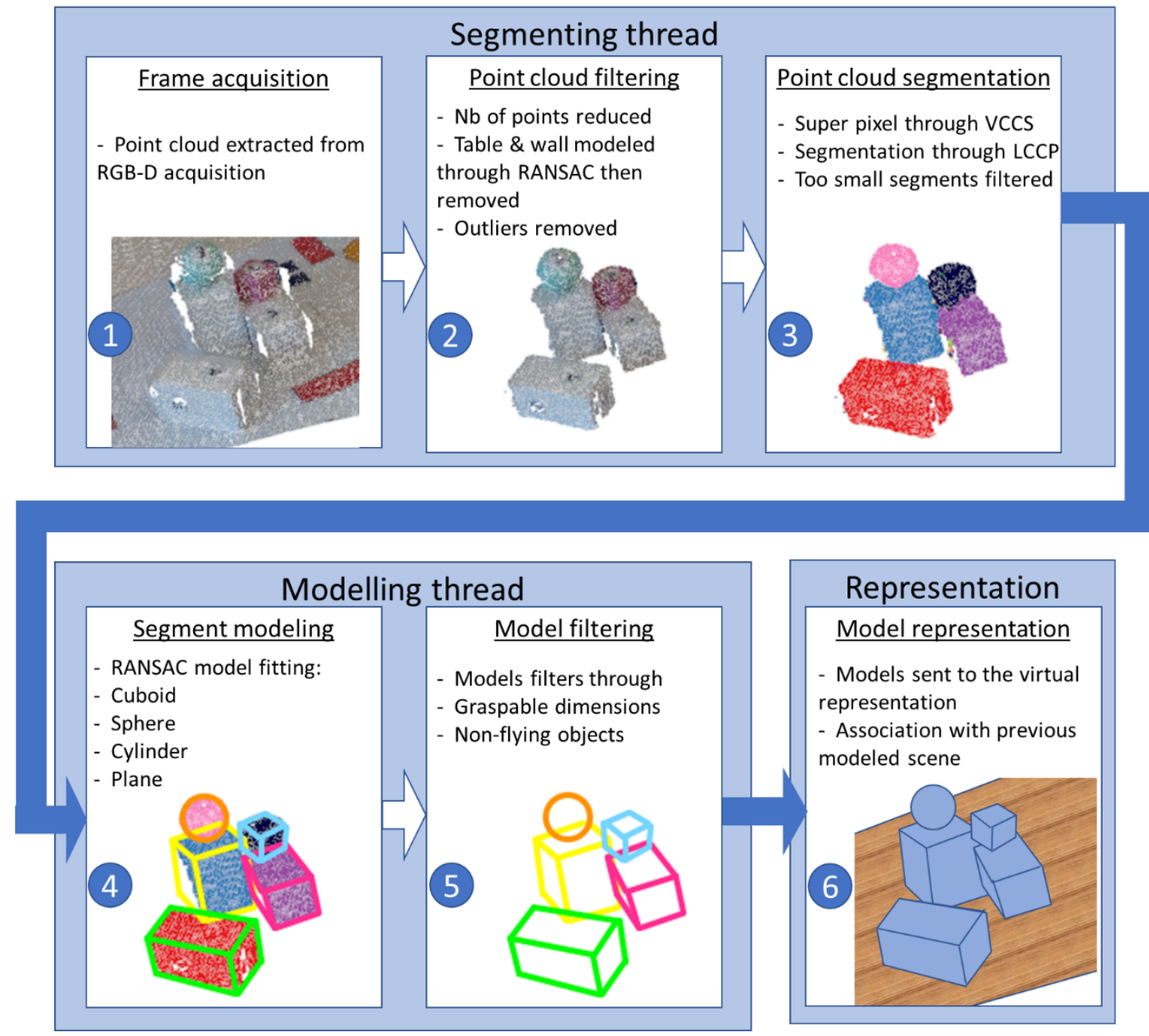

Figure 2.2 Scheme of the computer vision processing. The segmenting and the modelling threads are analysing the point cloud acquired from the RGB-D camera at $2 \mathrm{~Hz}$. The segmenting one 1) grabs the data from the camera, 2) filters the point cloud to remove the table, other big planes, and remaining single voxels, and 3) segments the remaining points using LCCP on supervoxels. Then a second thread 4) attributes to each segment the best fitting primitive using RANSAC, 5) filters the ungraspable models and sends it to Unity to 6) associate the model of this frame to the previous scene

The first thread focused on the initial processing of the data and the segmentation. A distance filter was implemented to remove points too far away or too close to the camera, based on the hypothesis that the grasp actions are happening at a maximal range of the extended arm of the user. The point cloud was then filtered to remove the outliers. The rotation of the camera was placing the table at the centre of the field of view. To isolate the graspable object, the best fitting planar surface was fit through random sample consensus (RANSAC) [111] to be removed from the scene and sent to the scene generation module. RANSAC is a brute force method that fits a pre-defined model such as a plane to random points in the point cloud. This operation 
Chapter 2: Automatization of the wrist and the hand based on the prediction of the intended object's grasped side

is done a large number of times, and the model that collides with the highest number of points (best quality) is retained. This method requires a relatively high amount of computational power, but this computational power is fixed and always results in a solution with its associated quality. Therefore, this algorithm has the advantage of not being affected by the scene's complexity and lets the choice to accept or not the proposed solution based on its quality. With a second iteration, the eventual part of the wall was removed if it occupied more than $30 \%$ of the remaining points. Depending on the position of the prosthesis, the voxels included in a cuboid centred on the socket and sharing the orientation of the prosthesis in the coordinate system of the camera were removed from the point cloud in order to avoid processing and modelling the prosthesis itself.

Once the point cloud was filtered, objects were segmented. To decompose objects in several parts to model each of these parts into independent primitives and separate different objects from each other, each cluster of remaining points was segmented into convex parts. Consequently, the supervoxel method Voxel Cloud Connectivity Segmentation (VCCS) [112] was applied on the whole filtered cloud to reduce the computing power. This method over-segments the point cloud in a regular manner in the 3D space and is considered state-of-the-art while being open-source [113]. The centroids of the supervoxels are placed on a regular grid in space. The affiliation of each voxel to one of these supervoxels is done from close to close via a connectivity graph and via a distance calculation taking into account the position, colour, and characteristics of the voxel's surface. This ensures a continuous oversegmentation, respecting the boundaries of the objects and requiring relatively little computational power. The locally convex connected patches (LCCP) method [114] is then applied to segment the different clusters into convex shapes. This method goes through every supervoxels to classify the connections with each of their neighbours as convex, concave or singular, according to their positions and their normal surface vectors. The labelling of each segment is then gradually spread to each supervoxel sharing a convex connection, and its voxels. The segments are therefore contained by concave or singular boundaries. This method relies on the supervoxels to respect the objects' boundaries and limits its need for computer power, making the combination 
Chapter 2: Automatization of the wrist and the hand based on the prediction of the intended object's grasped side

of the two methods usable online. It also has the advantage of splitting objects into graspable (convex) parts.

Each segment was modelled consecutively in the second thread by the different primitives using the RANSAC algorithm: plane, cube, sphere, and cylinder. The model with the best quality (ratio between the points included in the model and the total number of voxels in the segment) was retained. The primitive shapes were defined as:

- Plane: Standard plane detection, either perpendicular or parallel to the table. This primitive was used to filter out remaining parts of the wall and other ungraspable flat objects of the room,

- Cube: modelled by two to three planar surfaces perpendicular to each other and either perpendicular or parallel to the table.

- Sphere: standard sphere detection, with a minimum size of $3 \mathrm{~cm}$ diameter and a maximum size of $7 \mathrm{~cm}$.

- Cylinder: modelled by a tube whose axis was either parallel or perpendicular to the table and a planar surface perpendicular to the axis of the tube. 
Chapter 2: Automatization of the wrist and the hand based on the prediction of the intended object's grasped side

\subsubsection{Scene generation: short-term memory}

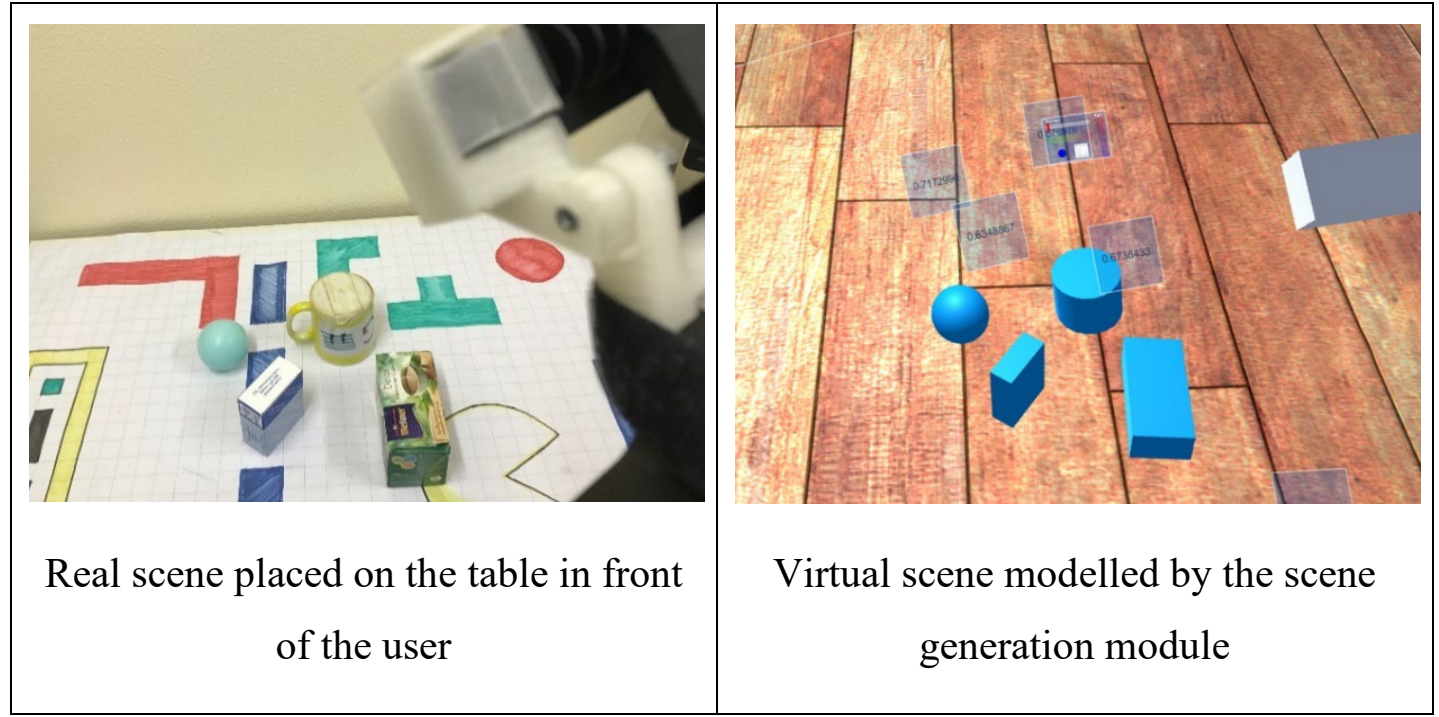

Figure 2.3 Comparison between the real scene and the generated scene. The camera is modelled as a white parallelogram, and the distance information hove above the objects. Both distance information, the modelled camera, and the modelled table were invisible to the user

The scene generating module gathered the information sent to the unit to place the different elements in the generated scene. Whereas the prosthesis, the camera and the table were single and continuously updated objects, the primitives transmitted by the artificial exteroception submodule at each frame were independent of the previous ones. Each new primitive modelled by the artificial exteroception module was associated with a possible existing model from the generated scene.

The system was designed to work in cluttered scenarios where different objects or different parts of objects were touching each other, implying potential modelling and association errors from one frame to the other. The different scenarios are presented in Figure 2.4. Due to errors from the sensor or poor modelling, future primitives and existing models may be missing when only some objects are correctly modelled or incorrect when the model does not represent any object in the real scene. In order to provide a stable and most accurate scene for the intention detection module to work with, the scene-generating module integrated median filtering over time. 
Chapter 2: Automatization of the wrist and the hand based on the prediction of the intended object's grasped side

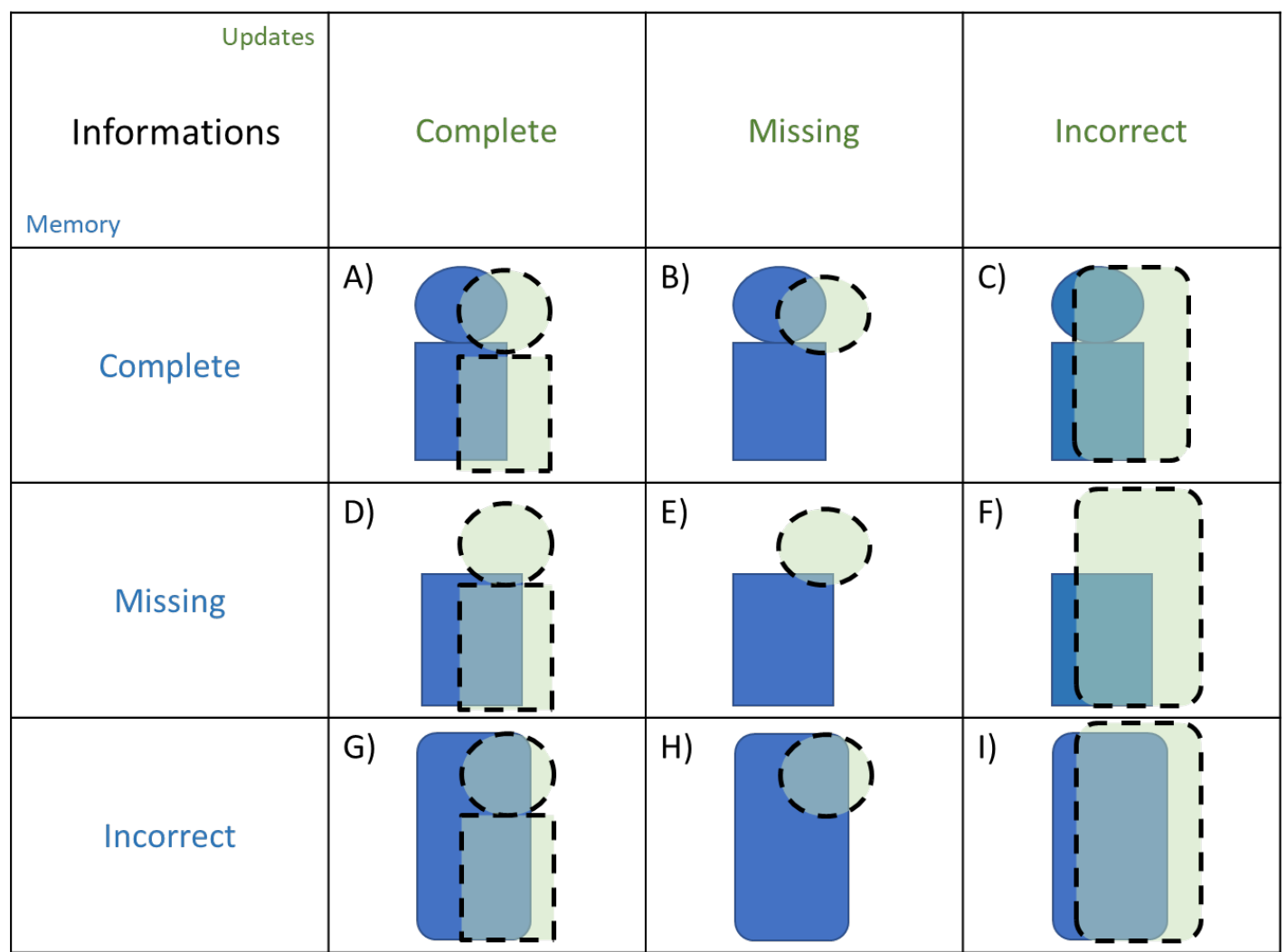

An algorithm illustrated in Figure 2.5 has been designed to associate the new primitive with the existing models and apply a median filter to answer this issue. This algorithm was based on the elections of representatives. Each object of the generated scene was associated with a working group. This working group contained the primitives associated with this object received during the ten last updates. At the end of each update, each working group computed the variance and the mean using Mahalanobis distance [115] of the variables of the primitives that it contained (position, dimensions, shape, orientation), weighted by their quality (the quality with which the RANSAC algorithm fitted the primitive to the point cloud segment). The primitive of the working groups closest to the calculated one was thus elected as the representative. The representatives of the working groups containing more than two primitives were visible in the generated world and taken into account during the intention detection. 
Chapter 2: Automatization of the wrist and the hand based on the prediction of the intended object's grasped side

The working groups were updated with each new image of the artificial exteroception submodule. The association algorithm aimed to associate the primitives and the working groups per pair, creating new groups for isolated new primitives. As such, the collisions between the new primitives and the primitives of each working group already present in the scene were first checked based on their coordinates and shape. If at least one collision was detected, the new primitive was added to the list of candidates of each working group with which it collided. The primitives which collided with only one working group were called single collision candidates, while the primitives which collided with several working groups were called multi-collisions candidates.

At each new frame primitives from the artificial exteroception submodule were checked for collisions, each working group selected the new primitive to integrate among its candidates, starting with the working groups with single collision candidates.

- If no collision was detected, a working group was created to gather the information for the newly detected object.

- When no candidate collided with the working group, an empty primitive was added to the group to report a missing update. After six misses in the ten last updates, the working group and all the models it contained were removed from the generated scene.

- When one or more new primitives collided with the group, the selection process started.

- If only one single collision candidate applied to this working group (situation illustrated in Figure 2.4 by the cases E, F, H, and I and by the cube of case A), this candidate was directly selected and was included in the "working group". If multi-collisions candidates had also collided with the working group, their number of collisions was reduced by one. If this reduction made them single collision candidates in another working group, the selection process of this other working group was launched. This process enabled to solve chains of conflictual 
Chapter 2: Automatization of the wrist and the hand based on the prediction of the intended object's grasped side

associations (see the conflictual association with sequential resolution in Figure 2.5)

- If several single collision candidates only applied to this working group (situations $D$ and $G$ ), the distance between them and the representative of the working group was computed through Mahalanobis distance, and the closest one was integrated into the working group.

- If only multi-collision candidates applied to the working group, illustrated in situation B from Figure 2.4, a recursive process first gathered all the working groups and multi-collisions candidates involved in the chain. Then the Hungarian algorithm [109] was applied to associate the candidates and the working groups per pair.

Eventually, new working groups were created for each remaining primitive. 
Chapter 2: Automatization of the wrist and the hand based on the prediction of the intended object's grasped side
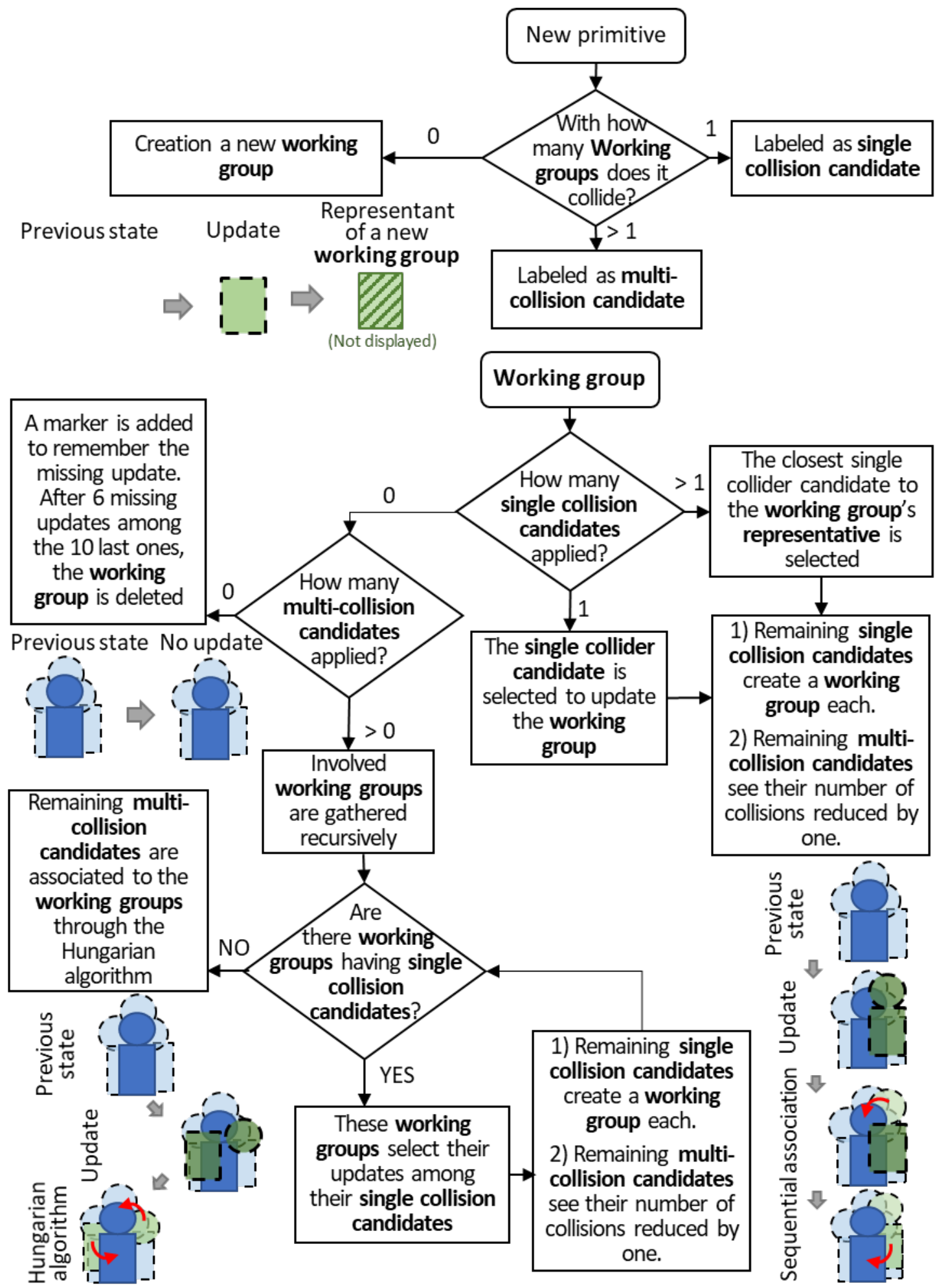
Chapter 2: Automatization of the wrist and the hand based on the prediction of the intended object's grasped side

\subsubsection{Intention detection and preshaping}

Based on the data provided by the scene-generating module, the intention detection module aimed to detect a grasping intention and determine which object was targeted by the user. This process was done by comparing the hand position with the representatives' position present in the generated scene. Only the representatives closer than $20 \mathrm{~cm}$ from the hand and not at more than $10 \mathrm{~cm}$ on the left of the prosthesis were considered. The grasping intention was triggered if a representative met these conditions while the hand had low speed (lower than $0.2 \mathrm{~m} / \mathrm{s}$ ). If only one representative met the conditions, it was selected directly. If several objects met these conditions, the selection was based on the position of the prosthesis grasping point as illustrated in Figure 2.6:

- If two objects were detected as stacked, only the object at the same height as the grasping point was considered

- In the other case, a coefficient (Selection $_{\text {Coef }}$ ) was computed inversely proportional to the distance between the grasping point and each representative involved, according to equation (2-7). The representative getting the highest coefficient was selected.

$$
\text { Selection }_{\text {Coef }}=\text { Hyst }_{\text {Coef }} *\left(1-\frac{\text { Dist }_{\text {HO }}}{{\text { MaxDistance }+ \text { Hyst }_{\text {Dist }}}_{\text {Distanction }}}\right)
$$

In this equation, Selection Coef $_{\text {is }}$ the Dist $_{H O}$ is the distance from the hand to the object, MaxDistance the maximum distance to be selected (here $20 \mathrm{~cm}$ ), Hyst $_{\text {Coef }}$ a hysteresis coefficient increasing if the object was previously selected. On the same model, Hyst $t_{\text {Dist }}$ increases the maximum distance to get selected by two centimetres if the object was previously selected while being null otherwise. This method allows a stable and real-time selection of the object of interest among a scene. 
Chapter 2: Automatization of the wrist and the hand based on the prediction of the intended object's grasped side
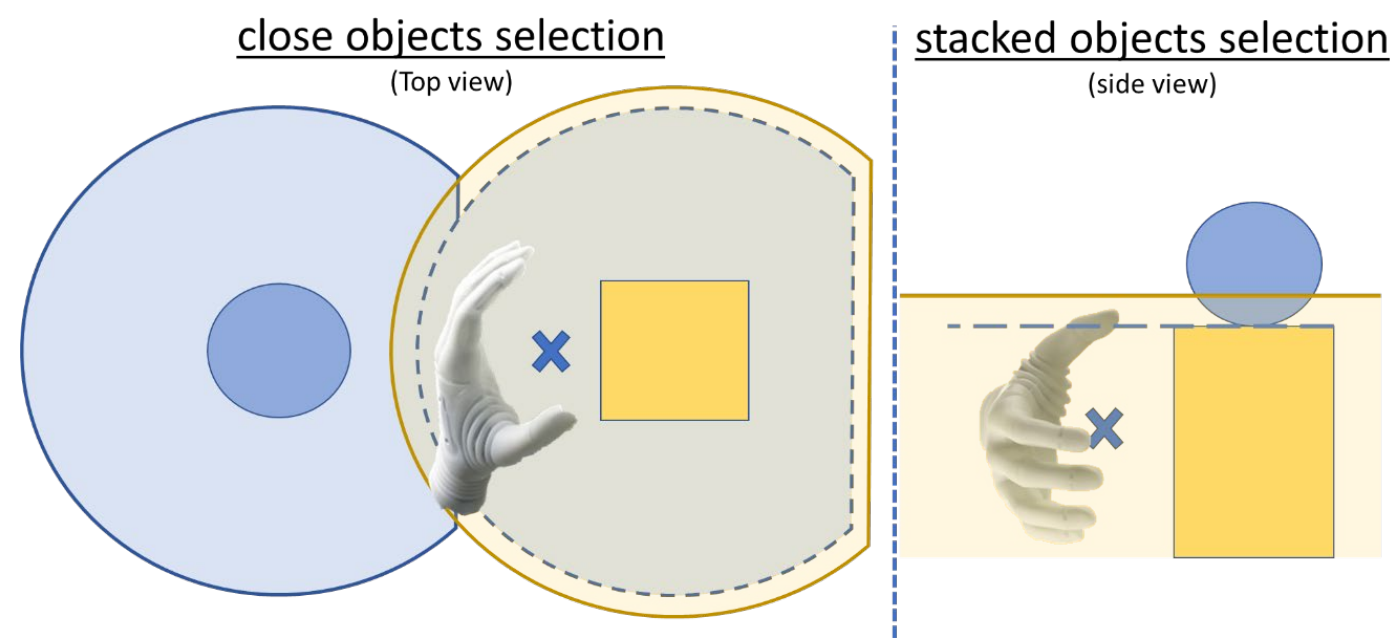

Figure 2.6. Illustration of the selection's areas. Selected objects are in yellow, unselected ones are blue. In the close objects' selection, the blue area represents the vicinity of each object. In both selections, the yellow area shows this vicinity increase by the hysteresis coefficient to stabilise the selection and avoid a flickering between two selection areas.

Once the intention of grasping was detected and the virtual object selected, the autonomous preshaping module computed and adjusted the orientation of the prosthetic wrist and fingers. Three grasp strategies were employed according to the hand's position relative to the object and the object's dimensions. The lateral grasp was employed if the object's face facing the user was smaller than three centimetres. The wrist then aimed to keep the hand parallel with the table. A variable of adjustment was added to the wrist's DoFs and adjusted each subject individually, mainly to compensate. The wrist flexion $\theta_{\text {flex }}$ and wrist rotation $\theta_{\text {rot }}$ were computed according to equations (2-8) and (2-9).

$$
\begin{gathered}
\theta_{\text {rot }}=\left|\theta_{\left(\overleftarrow{o_{u p}}, \overleftarrow{\left.P_{\text {rlght }}\right)}\right.}\right|-90^{\circ}+\theta_{\operatorname{Corr}_{\text {top-rot }}} \\
\theta_{\text {flex }}=90^{\circ}-\left|\theta_{\left(\overleftarrow{\left.o_{\text {up }}, \overleftarrow{P_{\text {forw }}}\right)}\right.}\right|+\theta_{\text {Corr }_{\text {top }- \text { flex }}}
\end{gathered}
$$

In these equations, $\theta_{\left(\overleftarrow{o_{u p}}, \overleftarrow{P_{\text {right }}}\right)}$ and $\theta_{\left(\overleftarrow{o_{u p}}, \overleftarrow{P_{\text {forw }}}\right)}$ are the angles between the vertical vector of the object's coordinate system and the right and forward vectors of the forearm. The variables $\theta_{C o r r_{\text {top-rot }}}$ and $\theta_{\text {Corr }_{\text {top-flex }}}$ are respectively the offsets for correction of rotation and flexion. 
Chapter 2: Automatization of the wrist and the hand based on the prediction of the intended object's grasped side

If the grasping point of the prosthesis was above the object, the top grasp strategy was selected, and the wrist aimed to keep the hand parallel to the table in the same way as the lateral grasp strategy. Lastly, in the remaining cases, the side grasp strategy was selected. The system oriented the wrist to place the palm perpendicular to the hand-object vector following these equations (2-10) and (2-11):

$$
\begin{aligned}
& \theta_{\text {rot }}=\left|\theta_{\overleftarrow{O_{\text {up }}, \mathrm{P}_{\text {right }}}}\right|-90^{\circ}+40^{\circ}+\theta_{\text {Corr }_{\text {side-rot }}}+0.12 \\
& *\left|\theta_{\left(\overleftarrow{P_{\text {forw }}}, \overleftarrow{\operatorname{Vec}_{H-O}}\right)}\right| \\
& \theta_{\text {flex }}=0.8 *\left|\theta_{\left(\overleftarrow{\left.P_{\text {forw }}, \overleftarrow{V e c_{H-O}}\right)}\right.}\right|-90^{\circ}+20^{\circ} \\
& +\theta_{\text {Corr }_{\text {side-flex }}}
\end{aligned}
$$

In these equations $\theta_{\left(\overleftarrow{P_{\text {forw }},}, \overleftarrow{V C_{H-O}}\right)}$ is the angle between the forearm's forward vector and the vector going from the hand to the object. The variables $\theta_{\operatorname{Corr}_{\text {side-rot }}}$ and $\theta_{\text {Corr }_{\text {side-flex }}}$ are the offsets for correction of the rotation and the flexion, adjusted for each subject individually $\left(-10^{\circ}, 10^{\circ}\right)$. The proportional gains of 0.12 and 0.8 in equations (4) and (5) were determined heuristically. 
Chapter 2: Automatization of the wrist and the hand based on the prediction of the intended object's grasped side

\subsection{Performances}

\subsubsection{Performance of the hand tracking}

The quality of the object selection and the preshape computation relied on the accuracy of the position of the prosthesis grasping point relative to the object of interest. Due to the design, previous exposed, of the hand tracking module, two factors needed to be considered: the accuracy of the distance between the reference point for the prosthesis tracking and the object or interest, and the accuracy of the orientation of the prosthesis adaptor used to compute the position of the prosthesis grasping point. According to Dr. Carfagni et al. (2017) [116], the camera SR300 enables to measure a distance of $39.5 \mathrm{~cm}$ with an accuracy of $6.05 \mathrm{~mm}$ in its working volume. Considering that the distance between the prosthesis grasping point and the prosthesis reference point was $29.5 \mathrm{~cm}$, the accuracy previously measured was appliable in the context of the autonomous controller implemented. An IMU MTw Awinda acquired the orientation of the prosthesis adaptor. The specifications of this sensor [117] claim an accuracy of $1.5 \mathrm{deg}$. The accuracy of the position of the prosthesis grasping point in relation to the object was then $14 \mathrm{~mm}$, given by (2-12):

$$
\begin{gathered}
A_{\text {Obj-Grasp }_{\text {Point }}}=A_{\text {Prosth }_{\text {Tracking }}}+D_{\text {Ref-Grasp }} \text { Point } * \sin \left(A_{\text {Orientation }}\right) \\
=6+295 * \sin (1.5)=14 \mathrm{~mm}
\end{gathered}
$$

In this equation, $A_{O b j-G r a s p}$ Point $_{\text {is }}$ is the accuracy of the position of the prosthesis grasping point in relation to the object, $A_{\text {Prosth }} h_{\text {Tracking }}$ is the accuracy of the distance between the reference point of the prosthesis tracking and the object of interest, $D_{R e f-G r a s p_{\text {Point }}}$, is the distance between the reference point and the grasping point of the prosthesis, and $A_{\text {Orientation }}$ is the accuracy of the orientation of the prosthesis adaptor.

\subsubsection{Performance of the object modelling}

The performance of the object modelling has been assessed through two measurements: the stability of the object's position and the accuracy of its size. These variables have been measured during a static test of 30 seconds during which five 
Chapter 2: Automatization of the wrist and the hand based on the prediction of the intended object's grasped side

objects (two CD boxes, one small box, one sphere and one tee box) were placed in front of the camera spread in the working area of the autonomous controller (see Figure 2.7).

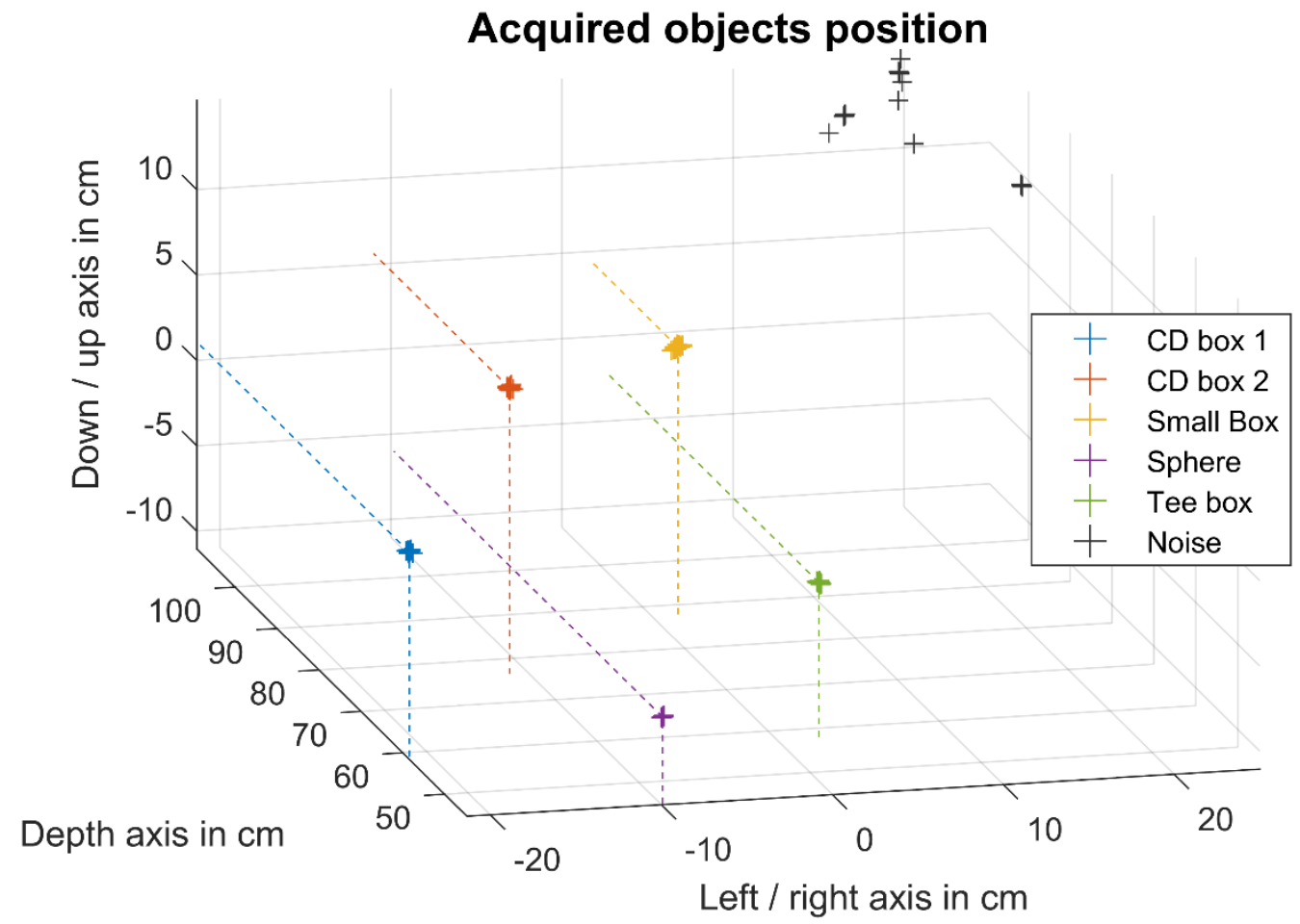

Figure 2.7 Acquired objects position during the assessment of the object modelling module performances. The objects were placed on a table. The point of view of the graph would be above on the right of the camera.

The deviation of the position of each object is given in Figure 2.8. The furthest object from the camera, the small box had the highest median deviation of $1.7 \mathrm{~mm}$ and the highest maximal deviation of $6.0 \mathrm{~mm}$. 
Chapter 2: Automatization of the wrist and the hand based on the prediction of the intended object's grasped side

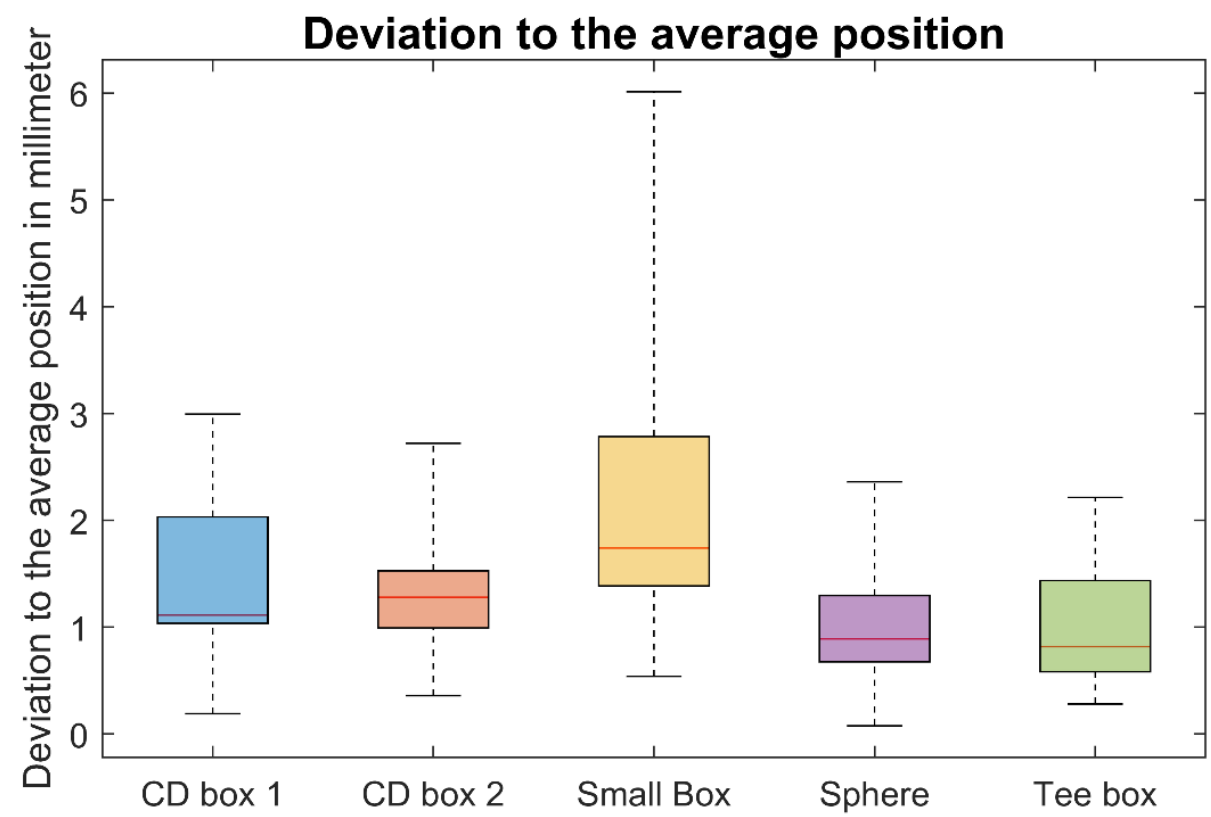

Figure 2.8 Deviation to the average position for different objects at five different positions.

Last, the measured size of the modelled objects is illustrated in X. The worst accuracy has been measured for the first CD box with a median error of $8.0 \mathrm{~mm}$.

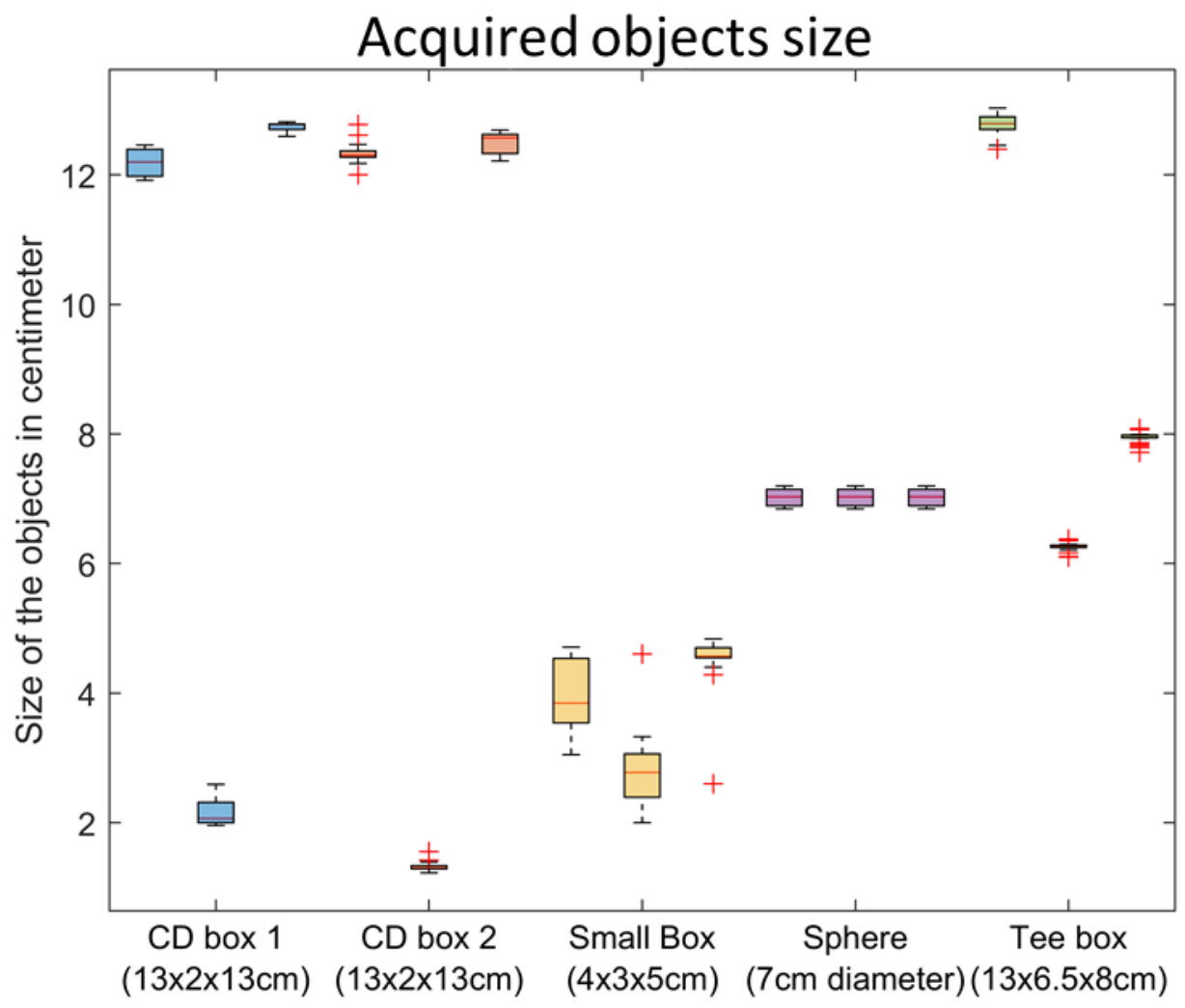

Figure 2.9 Acquired objects size during the assessment of the object modelling module. 
Chapter 2: Automatization of the wrist and the hand based on the prediction of the intended object's grasped side

\subsection{Conclusion}

The method developed in this chapter enables the autonomous preshaping of the hand when performing and reach-to-grasp task. Its design aims to answer the main limitations affecting the autonomous systems presented in the literature.

Based on the hand's position relative to the part of the object rather than its shape to predict the user's intention, the developed autonomous system follows the grasp strategy that the user implements. The object segmentation into convex parts enables the target of the particular part of the object close to the hand, allowing the user to grasp the body as the handle according to the object's disposition. The automation resulting from the prediction includes the two grasp-types from the hand and actuates the two DoFs of the wrist.

Because the automation of the hand is only conditioned by the proximity of the hand to a graspable object and the low speed of the hand, it does not require an additional action when performing a reach-and-grasp task. On the other hand, once in the vicinity of the object, the prosthesis's automatic preshaping follows the user's movement in real-time, adapting to the new predicted object or new predicted side. The accuracy of the estimation of the position of the prosthesis grasping point in relation to the object of interest is high enough to accurately determine a suitable preshaping position when the hand is at a small distance from the object. The offset of $1.5 \mathrm{~cm}$ added on the aperture of the hand prosthesis compensate for the median error of the object size estimation.

By including the input of the hand's position in regard to the object provided by the user, the autonomous controller aims to adapt to the grasping strategy of the user rather than to require the user to use one single solution. The autonomous controller handles the selection of the grasp type, the adjustment of the opening, and the two DoFs of the wrist to preshape the entire prosthesis. 
Chapter 2: Automatization of the wrist and the hand based on the prediction of the intended object's grasped side

\subsubsection{Current technical limitation}

While the prototype developed for this project answers the technical limitations previously identified, it still comprises elements limiting its usability.

Firstly, the system is still difficult and uncomfortable to wear. The Creative SR300 camera, a core component of the system, is relatively large and heavy. This component is relatively old in regard to the progress of technology and already has new versions providing better resolution [118] for smaller weight and better comfort.

Secondly, the range of applicable situations remains limited. The IR camera is sensitive to changes in lighting and sudden over-exposure due to direct sun exposure of the object to sunlight. All tests and applications were carried out under controlled lighting conditions to stabilise the performance of the autonomous controller. The technology chosen for camera tracking and its relatively low performance limits the use of this system to a small area around the calibration frame. This could potentially be improved by resetting the ego-motion periodically. The computer may also be replaced by using cloud computing and wireless transmission, which has already been implemented in similar projects [79], [89], [119], [120].

The understanding of the scene is also limited. Due to the camera's resolution, the objects or parts of objects smaller than one $\mathrm{cm}^{3}$ are not modelled. This may be solved in the future by embedding a better camera. The modelling of the objects is based on single frames obtained from the RGB-D camera and are therefore point clouds projected from a single point of view. This process can be sensitive to noise and requires post-processing like the median filter applied in this system. It also affects objects particularly sensitive to single projections, such as highly concave objects. The body of cups may be segmented into two different parts: the front and the back parts of the tube. It might be interesting to change the technology to a point cloud-building simultaneous localisation and mapping (SLAM) algorithm such as ORBSlam [121], [122], which would both enable better camera tracking over a much larger area and provide a point cloud from different camera positions. Such a point cloud would enable a finer segmentation and understanding of objects, for example [123]. 
Chapter 2: Automatization of the wrist and the hand based on the prediction of the intended object's grasped side

Finally, the computation of the hand preshape currently only includes data on the orientation and the size of the object, with no prior knowledge associated with it. This solution adapts to the user's grasping strategy to benefit from their knowledge of the object. Typically, the method proposed here does not differentiate between the function of the head and the hammer's body. This is not a critical issue because it is the user's role to place the hand in a graspable position to grasp the hammer's handle. This implementation allows the prosthesis to grasp the head if it is the intention of the user. However, the inclusion of the body part function could increase the probability of targeting the handle in case of uncertain prediction. In addition, adding information or "experience" to a body part can also enable specific grasping strategies to be forced when selected. This could facilitate the following manipulation: grasping a mug by the handle may require a particular shape of the finger, such as lateral grasping instead of a palmar grasping by default selected if the handle is on the side of the mug (and not in front of it). Ideally, these specific grasps should be individualised. 
Chapter 3: Assessing the support provided by an autonomous controller when combined with an ML controller

\section{Assessing the support provided by an autonomous controller when combined with an ML controller}

Several studies have already assessed the ability of an autonomous controller to support the user during specific tasks. However, as we have seen previously, the autonomous controller was not always integrated into an SA system allowing the user to interact with the prosthesis during the task. Moreover, no SA system has been compared to an advanced myo-control giving the user more flexibility to control all the DoF of the prosthesis.

Therefore, this chapter aims to investigate if the support of an autonomous controller can improve the performance of a prosthesis user using an ML myocontroller during a task including reach-and grasp actions. Since the SA system relieves the user of actuating the prosthesis' DoFs during the preshaping phase of the movement, reduced use of the myo-controller interface is expected. Finally, since the autonomous controller is only involved during the preshaping phase, this specific phase should have a shorter duration and lower use of myo-control from the user. 
Chapter 3: Assessing the support provided by an autonomous controller when combined with an ML controller

To assess these hypotheses, the autonomous controller previously developed has been embedded into a human-machine interface comprising a pattern recognition myo-controller and a feedback module to improve the symbiosis between the two agents in control of the prosthesis. The outcomes obtained using this SA system have been compared with the ones obtained using only the myo-controller. Those outcomes were obtained through two different experiments, the custom clustered scene interaction (CSI) and the standard functional outcomes measurement SHAP, assessing the system under different conditions reproducing more complex ADLs than the ones used to assess the previous SA systems.

\subsection{Implemented Human Machine Interface}

In order to combine the autonomous controller with the manual controller so that the user can control the prosthesis, the autonomous controller needs to be integrated into an SA system. This system comprises the autonomous controller previously developed, a myo-controller, a shared control module responsible for the distribution of the control of the prosthesis, and finally, a feedback module aiming to improve the symbiosis between the user and the autonomous controller. 
Chapter 3: Assessing the support provided by an autonomous controller when combined with an ML controller

\subsubsection{Myo-control}

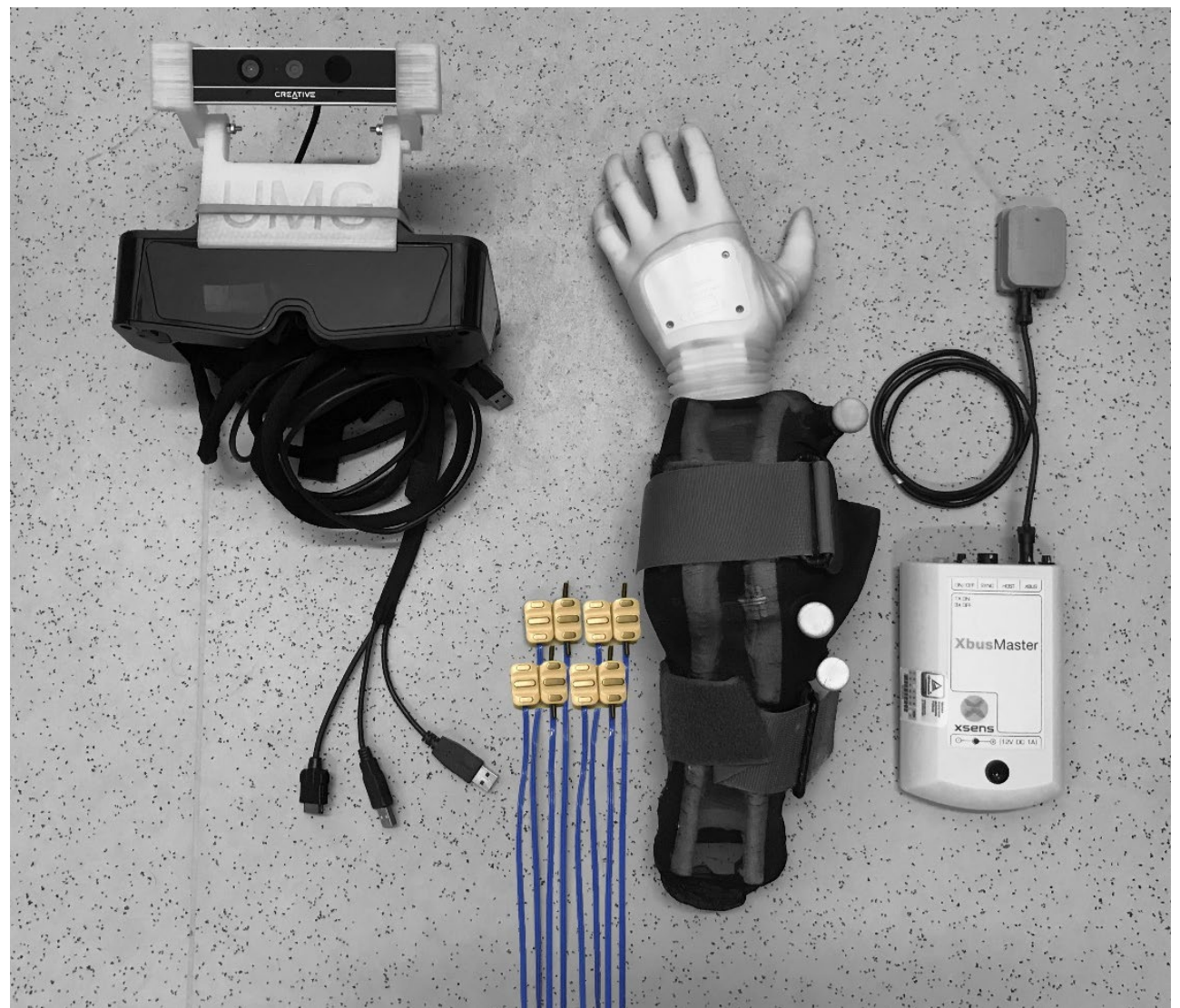

Figure 3.1 Photography of the material used during the experiment with the highlight of the electrodes. Eight DC electrodes were used to acquire the EMG signals around the forearm of the participant.

During the whole task, the user could control the prosthesis using muscle contractions. The signals provided by the eight electrodes were processed in Simulink. Eight EMG channels were acquired by the eight DC electrodes illustrated in Figure 3.1. The signal acquired were sampled at $100 \mathrm{~Hz}$ and classified through an LDA algorithm into either seven classes for the able-body subjects (close lateral, close palmar, open, pronation, supination, flexion, and extension), or four classes for the amputee subjects not able to perform seven different muscle patterns. Those amputee participants could switch from wrist control to finger control thanks to a short contraction in specific channels selected during the training session. This way, even being limited by the number of muscle contraction patterns they could produce, amputees could directly control the grasp or the wrist. 
Chapter 3: Assessing the support provided by an autonomous controller when combined with an ML controller

\subsubsection{Shared control modality}

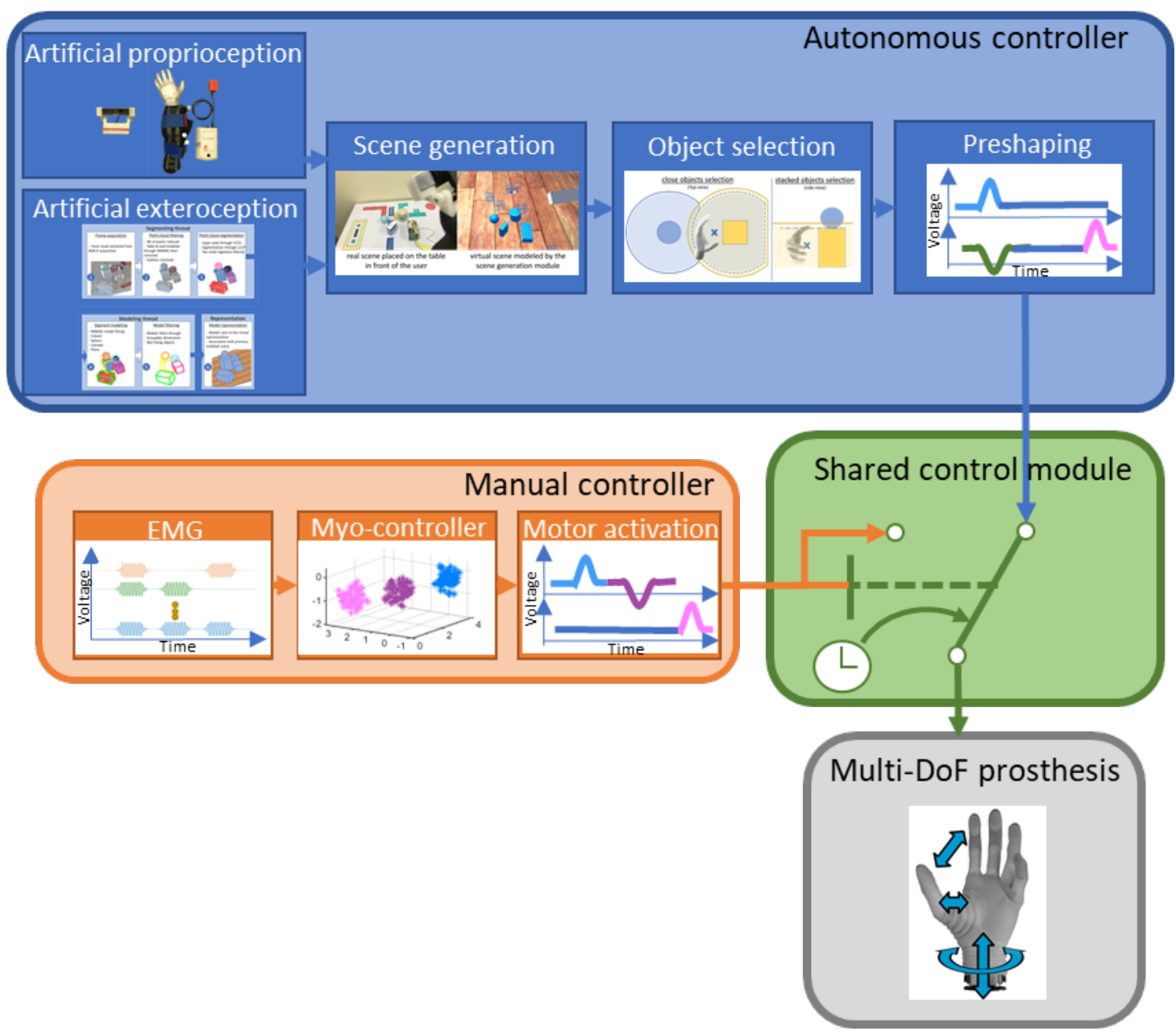

Figure 3.2 Scheme of the control part of the SA system. The autonomous and the manual controllers' outputs are merged in the shared control module to command the prosthesis

The shared control module selected the source of the control commands sent to the prosthesis through a sequential shared control modality (traded control) to merge the manual and the autonomous controller signals. The user's commands had priority over any commands from the autonomous preshape module in order to remain a support device and to ensure the direct application of the user's will. When a muscular contraction was detected, the command output of the LDA classifier was sent to the prosthesis, and the commands from the autonomous preshape module were ignored. After a window of inactivity from the user (one second), the commands from the autonomous preshape module were retaken into account. The same behaviour is produced as long as one object is grasped to ensure the user's total control of the hand. 
Chapter 3: Assessing the support provided by an autonomous controller when combined with an ML controller

This sequential control enabled to lock off the autonomous control when movements of the hand were too dangerous, such as when the hand was too close to several objects, or to ensure that the current pose of the prosthesis enabling the grasp does not change during the last centimetres of the reaching phase.

\subsubsection{Feedback Module}

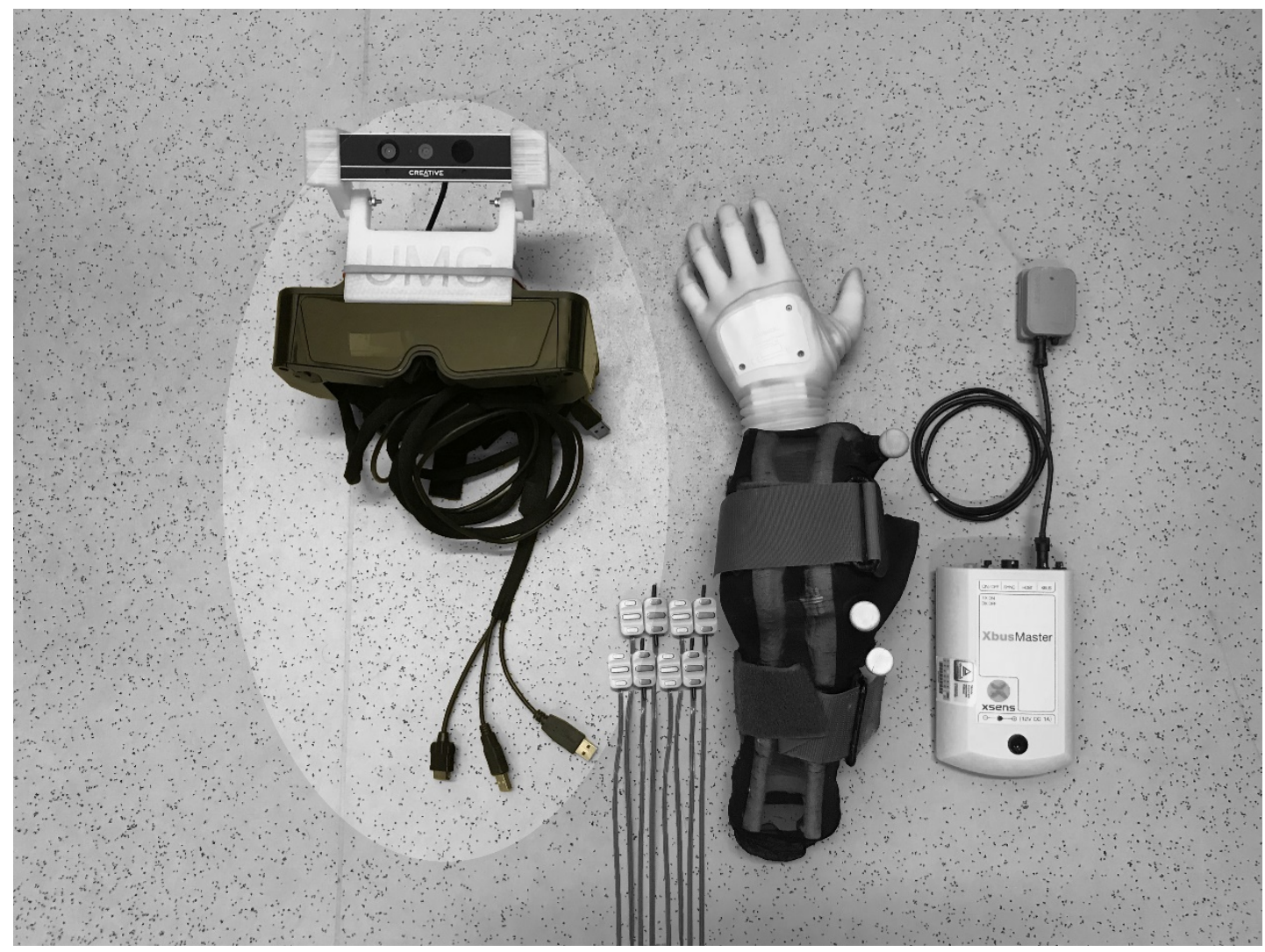

Figure 3.3 Photography of the components used with highlight of the AR Glasses. Those were connected to the computer through an HDMI cable

To provide information from the system to the user, the feedback interface displayed visual information through the AR Glasses illustrated in Figure 3.3. The virtual objects detected by the system were superposed to the user's vision at their computed positions. The colour of these objects indicated the selection and the decision taken by the system. While the shapes were modelled blue when the system planned no interaction, the objects were coloured yellow if selected. According to the grasping decision of the autonomous preshaping module, a green indication appeared on the object. 
Chapter 3: Assessing the support provided by an autonomous controller when combined with an ML controller

- If a top-grasp intention was detected, the top side of it turned green.

- If the decision was a lateral grasp, a green vertical band surrounded the object.

- If the decision was a side grasp, a green horizontal band surrounded the object.

Icons were displayed in the corners to indicate to the user the state of the system. A blue 2D box icon in the left top corner indicated that the scene modelling module is active. In the right top corner, two icons indicated the decision state:

- a yellow circle indicated that the system was selecting an object and adapting the prosthesis to grasp it,

- a green hand indicated that the system was off because of manual commands detected or because of an object in the hand.

- No icon appeared if the hand was too far away from any object of the modelled scene.

An example of a reach, grasp, and relocation action is given in Figure 3.4 with the user's vision through the AR glasses.

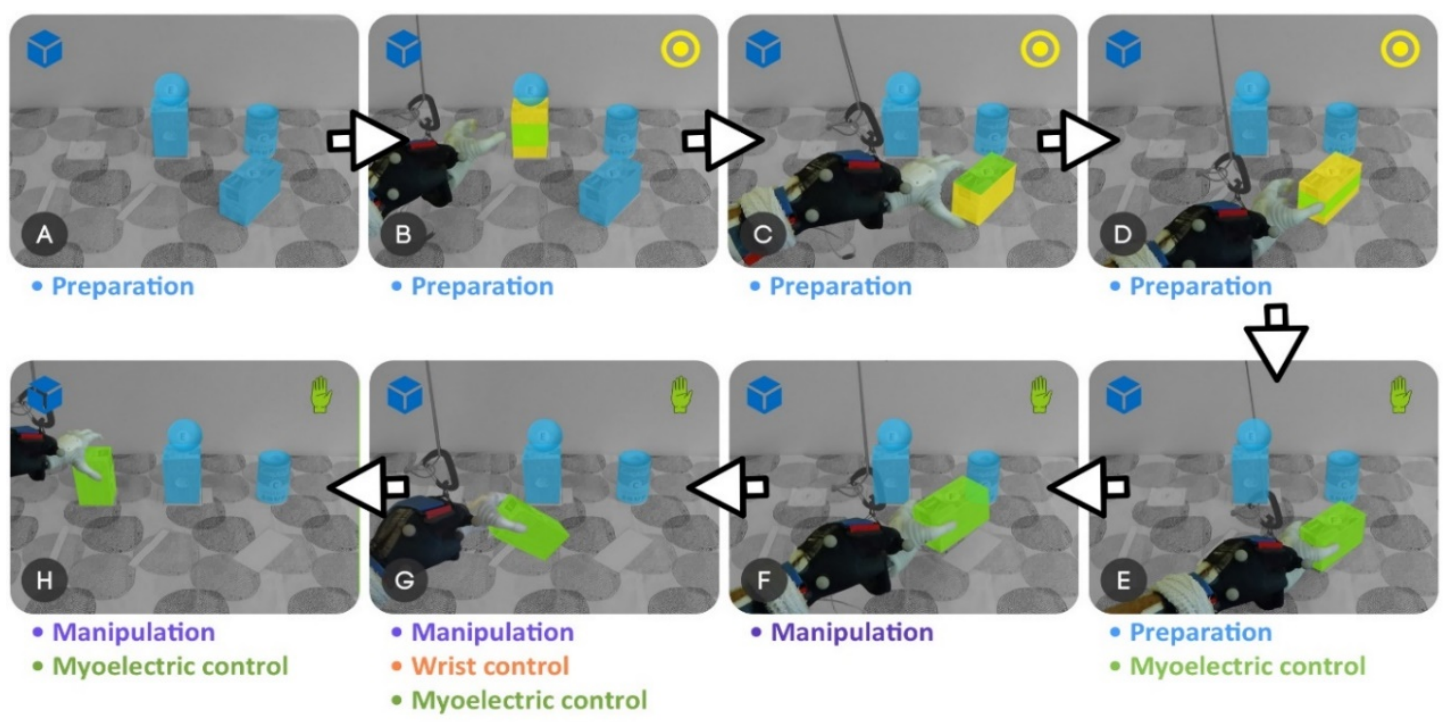

Figure 3.4 Illustration of the process of a grasp with detail of the different phases and representation of the feedback provided to the user through AR glasses , from [124] 
Chapter 3: Assessing the support provided by an autonomous controller when combined with an ML controller

\subsection{Experimentation}

Several standard protocols exist in upper-limb rehabilitation to assess the dexterity of individuals using a prosthesis [125]. In order to answer the hypotheses, the experiment was required to comprise an object-crowded scene, a sequence of manipulation, a diversity of objects, and ADLs. As no functional outcome measurement embedding all these requirements was found, the first experiment, called clustered scene interaction (CSI), was developed to confront the participant with a scene comprising a relatively high density of objects placed on a table. The task was to perform a relocation sequence designed to require a variety of grasps. The second experiment was a part of the standard and popular Southampton hand assessment protocol SHAP [126]. This protocol is divided into two parts: the abstract objects, requiring the participant to replace six objects of different forms and weights, and the ADLs reproducing fourteen manipulations from a daily life routine. This protocol has the benefit of combining different objects' shapes supposed to represent the variety of shapes present in everyday life and grasp strategies conditioned by the following manipulation.

The performance was expected to be highly impacted by the learning curve because of the novelty of the different components for the user to adapt. In order to reduce the effect of this learning curve and fatigue, the CSI experiment was conducted in three sessions consisting of a two-hour training session and one session for each condition in random order. The training session introduced the able-body and the amputee subjects to the myo-control, the autonomous controller, and the task.

\subsubsection{System Initialisation}

Before each session, the system was calibrated. First, the myoelectric interface was calibrated in two different arm positions (shoulder $20^{\circ}$ extended and elbow $70^{\circ}$ flexed, shoulder $75^{\circ}$ extended, and elbow fully extended). Three steps were necessary for the calibration: 1) measurement of the isometric maximal voluntary contraction (MVC), 2) collection of the data for the training of the classifier, done by asking the subject to perform the movement at two contraction levels ( $40 \%$ and $80 \%$ of the MVC) and 3) the fine-tuning of each threshold and each gain to regulate the class detection. 
Chapter 3: Assessing the support provided by an autonomous controller when combined with an ML controller

At the end of this process, amputee subjects were first tested on their ability to produce six different muscle patterns. If they were not satisfied with the prosthesis controllability, they tested the four-class control.

The calibration of the SA system required the calibration of the AR glasses, the IMU, and the adaptation of the autonomous preshapes. Next, the IMU was calibrated by placing the three IR markers in the camera's field of view to compute the orientation of the constellation and the relation between the coordinate system of the IMU and the camera, as developed in section 2.1.2. Last but not least, the autonomous preshapes were fine-tuned by slowly adjusting the parameters, whereas the participant put their hand in the grasping condition.

\subsubsection{Protocol of the tasks}

Eight male right-handed non-disabled participants (from 23 to 30 years old) and two amputee subjects using transradial myoelectric prostheses (59 and 61 years old) participated in the experimentations. All the participants signed a written consent form approved by the Ethical Committee of the University Medical Centre Göttingen $(22 / 04 / 16)$. Three non-disabled subjects had previous experience in myoelectric control. Both amputees regularly used transradial myoelectric prostheses.

The first test, CSI, performed by the amputee and the able body participants, has been designed based on the "abstract object" of the SHAP, requiring the participant to relocate sequentially different objects. To get closer to a daily life situation and to assess the performance of the system in challenging conditions, the CSI extended it by placing several objects on the table during the whole task. Those included two cd boxes $(130 \times 20 \times 130 \mathrm{~mm})$, two tea boxes $(130 \times 65 \times 80 \mathrm{~mm})$, one can ( $117 \mathrm{~mm}$ height, $65 \mathrm{~mm}$ diameter) and one plastic peach (around $75 \mathrm{~mm}$ diameter). The CSI also extended the SHAP by requiring an uninterrupted sequence of action to the subject. The purpose of this sequence was to assess the potential benefits of the SA system in a changing scene. The participants performed the task standing, which allowed them to utilise a wider variety of motions and body postures. As illustrated by Figure 3.5, the sequence of relocation was fixed. The participant had to put the two $\mathrm{CD}$ boxes on the left, then move the can and the peach on top of it on the marked 
Chapter 3: Assessing the support provided by an autonomous controller when combined with an ML controller

position on the right. For the fourth manipulation, the participant had to turn the tea box of $90^{\circ}$, then put the peach on it. Eventually, the last tea box was placed vertically close to the first one. Due to the limited field of view of the AR glasses, this sequence of manipulation required the user to orient the head to follow the object and therefore move the camera. The participants were also encouraged to grasp the objects as they preferred, which required the autonomous control to react to the possibly inconsistent behaviour of each participant. An oral countdown was given to start the trial, and the release of the last object was marking the end.
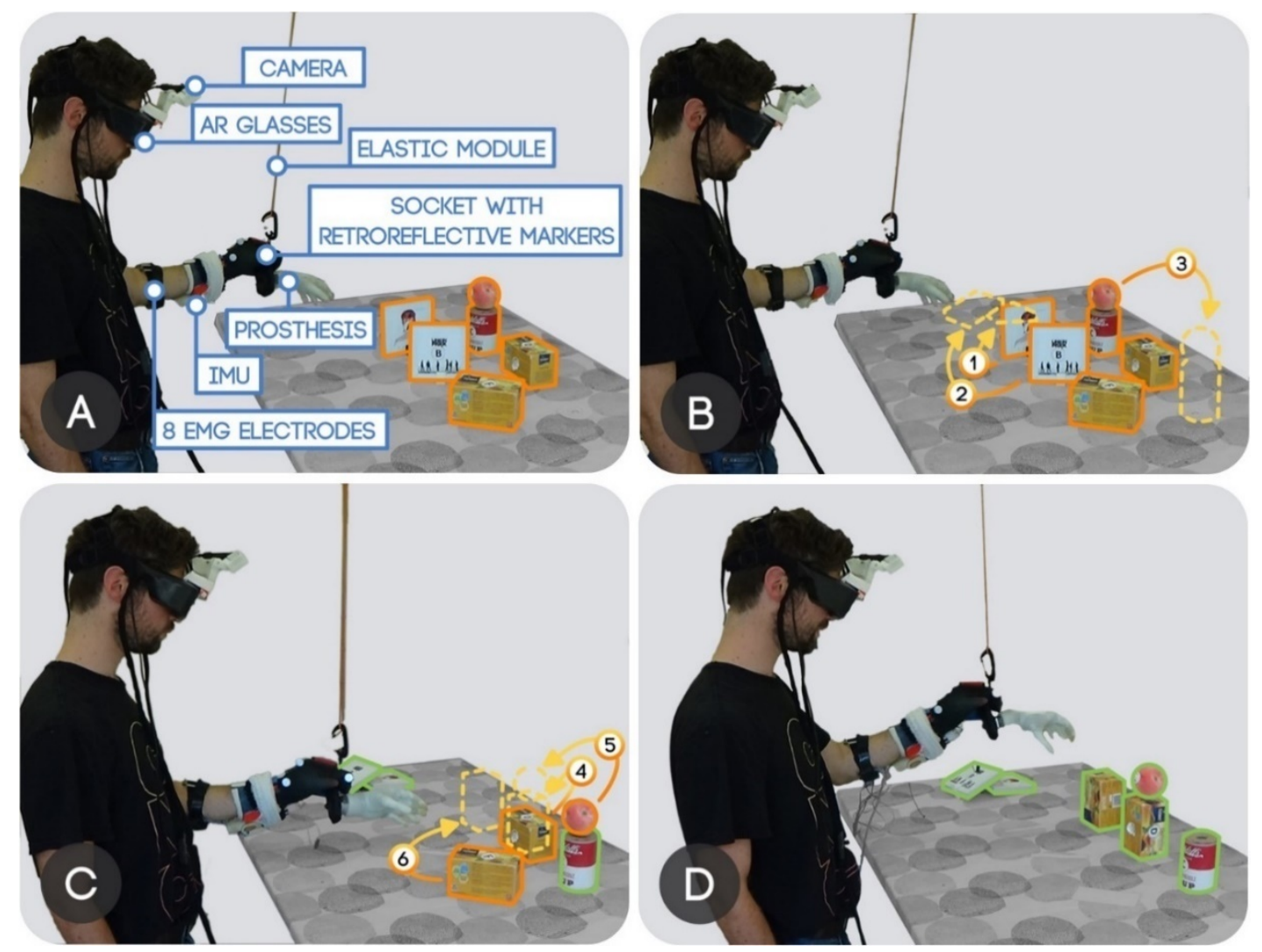

Figure 3.5 Overview of the Cluttered scene interaction test (CSI) from [124]. The experimental setup and the task sequence are here represented. (A) the initial position of the user and the objects. The elastic is fixed to a pulley allowing left/right movements and compensate for the weight of the prosthesis (B) repositioning of the $2 C D$ boxes $(1,2)$ and of the objects-set (3) \{tin can, peach\}. (C) Rotation of the first tee box (4), translation of the peach from the top of the tin can to the top of the tee box (5), and transfer of the second tee box (6). (D) Final position of the objects at the end of the trial.

To extend the assessment to more complex objects used in daily living, seventeen out of twenty-five tasks of the SHAP test were carried out by the amputee participants. These included every abstract object's relocation and the daily life 
Chapter 3: Assessing the support provided by an autonomous controller when combined with an ML controller

activities such as "Jar Lid”, “Glass Jug Pouring”, “Carton Pouring”, "Lifting a Heavy Object", and "Lifting a Light Object". The remaining seven tasks were excluded because of the incompatibility with the current setup (small object sizes, highly reflective objects, ...). Following the official instruction, the trial started and ended with the participant's push-button activation.

Participants completed ten trials per condition during the CSI test. Following the manual, the tasks from the SHAP test were not repeated. No time limit was set, but participants were instructed to perform it as fast as possible while at the same time avoiding excessive compensatory movements. No instructions were given regarding how to grasp or manipulate the objects. The experimenter observed the task execution, and the trial was repeated

1) if, when using the SA system, the subject continuously used manual commands, which prevents the autonomous system from sending any command,

2) if the user performed excessive compensatory movements;

3) if the subject dropped an object while performing the trial;

4) if hardware stopped functioning (e.g., the camera froze), before $5 \%$ of the trials had happened.

\subsubsection{Data Analysis}

The time required to complete the two experimental tasks (total time) was compared between the two conditions (the SA system versus. LDA) to assess the hypothesised increase of performance in accomplishing the tasks using the SA system compared to LDA. Therefore, this time measured from the start of the trial until its completion was the primary outcome measure in the CSI and SHAP test.

As it was also hypothesised that the SA system also decreases the overall use of myo-control, the time during which the user-generated myoelectric inputs to control the prosthesis was recorded (myo-control time) and then compared as a secondary outcome measure. The myo-control duration was the cumulation of all the intervals in which the user-generated myoelectric control inputs in any trial phase. This time was again divided into hand (i.e., aperture) and wrist control (flexion and rotation). 
Chapter 3: Assessing the support provided by an autonomous controller when combined with an ML controller

Again, it was expected that the SA system would only impact the grasp preparation duration (preparation time) without affecting the manipulation duration (manipulation time). Indeed, the system was not active during this last phase. Therefore, the evolution of the two outcome measurements in each phase was compared between the two conditions. The manipulation time was the time interval between the grasping of the object and the subsequent object release. Since the autonomous system supported the preshaping of the prosthesis before the grasp, we expected a decrease in grasp preparation time with an unchanged manipulation time when using the proposed system.

For the CSI test, the mean values of each outcome measure were compared between the interfaces (the SA system versus LDA). For the SHAP test, task durations passed the Kolmogorov-Smirnov normality test and were therefore compared with a paired t-test. The rest of the variables did not pass the Kolmogorov-Smirnov normality test. Therefore, the non-parametric Wilcoxon signed-ranks test was used to evaluate statistically significant differences. The results of the CSI test are reported as median (inter-quartile range); those of the SHAPs tests are reported as cumulative duration of the tasks. The significance level was set at $p<0.05$.

\subsection{Results}

One hundred and sixty trials ( 2 control methods x 10 trials $\mathrm{x} 8$ subjects) were performed during the CSI, test by non-disabled and 32 (2 control methods $\mathrm{x} 8$ trials $\mathrm{x}$ 2 subjects) by amputee subjects. Only the last six trials were used to minimise the influence of training on the performance outcomes. All subjects successfully performed the tasks in both the SA system and LDA conditions. 
Chapter 3: Assessing the support provided by an autonomous controller when combined with an ML controller
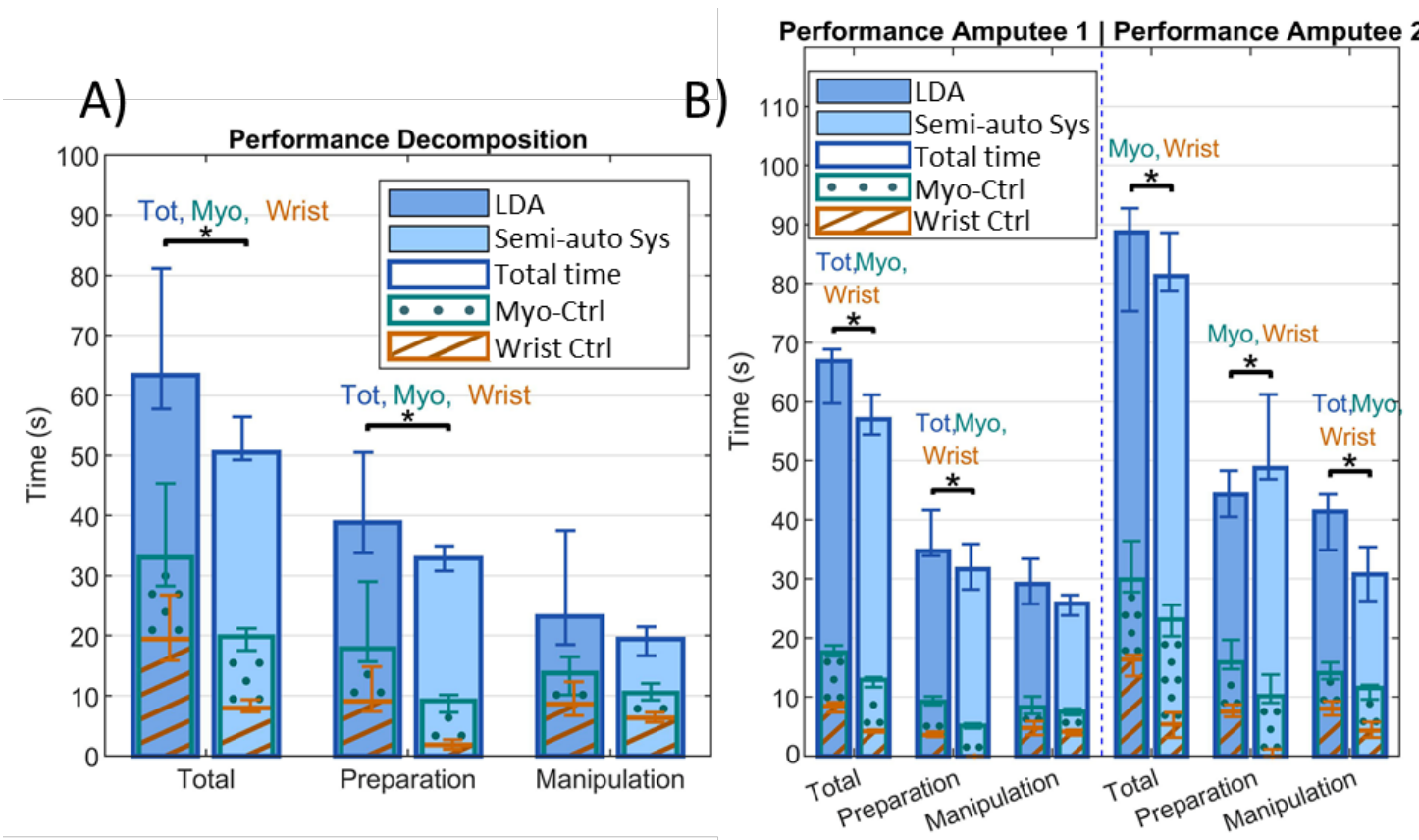

Figure 3.6 Trial time decomposition for the cluttered scene interaction (CSI) test performed by both able participants (A) and by amputee participants (B). The trial execution time and the total time spent using myocontrol are decomposed in preparation and manipulation phases. The indications above the asterisks stand respectively for a significant difference $(p<0.05)$ in the total amount of time (Tot), the time spent controlling the prosthesis manually (Myo), and the time spent controlling the wrist manually (Wrist) for each phase of the task.). From [124]

Concerning the able-body group, the total trial time was significantly longer for LDA (63.3 [23.4] seconds) than for the SA system (50.5 [7.2] seconds), indicating a better overall task performance with the SA system. In detail, only the preparation time was significantly different, reducing from 38.0[16.8] seconds when using LDA to 32.9 [4.2] seconds when using the SA system. The time spent by the subjects generating myo-control activities also reduced significantly with a total reduction of the myo-control time from 34.6 [18.3] seconds using LDA to 22.6 [6.7] seconds using the SA system. This significant difference was found in the preparation phase, where subjects reduced myo-control time from 17.9 [13.2] seconds with LDA to 9.1 [2.9] seconds with the SA system. Concerning the target of the control, the time spent on the wrist control was significantly smaller for the SA system during both grasp preparation time and total trial time, while no significant difference was observed for the aperture control. The interquartile range for the total duration time was reduced from 23.5 seconds for LDA to 7.2 seconds for the SA system 
Chapter 3: Assessing the support provided by an autonomous controller when combined with an ML controller

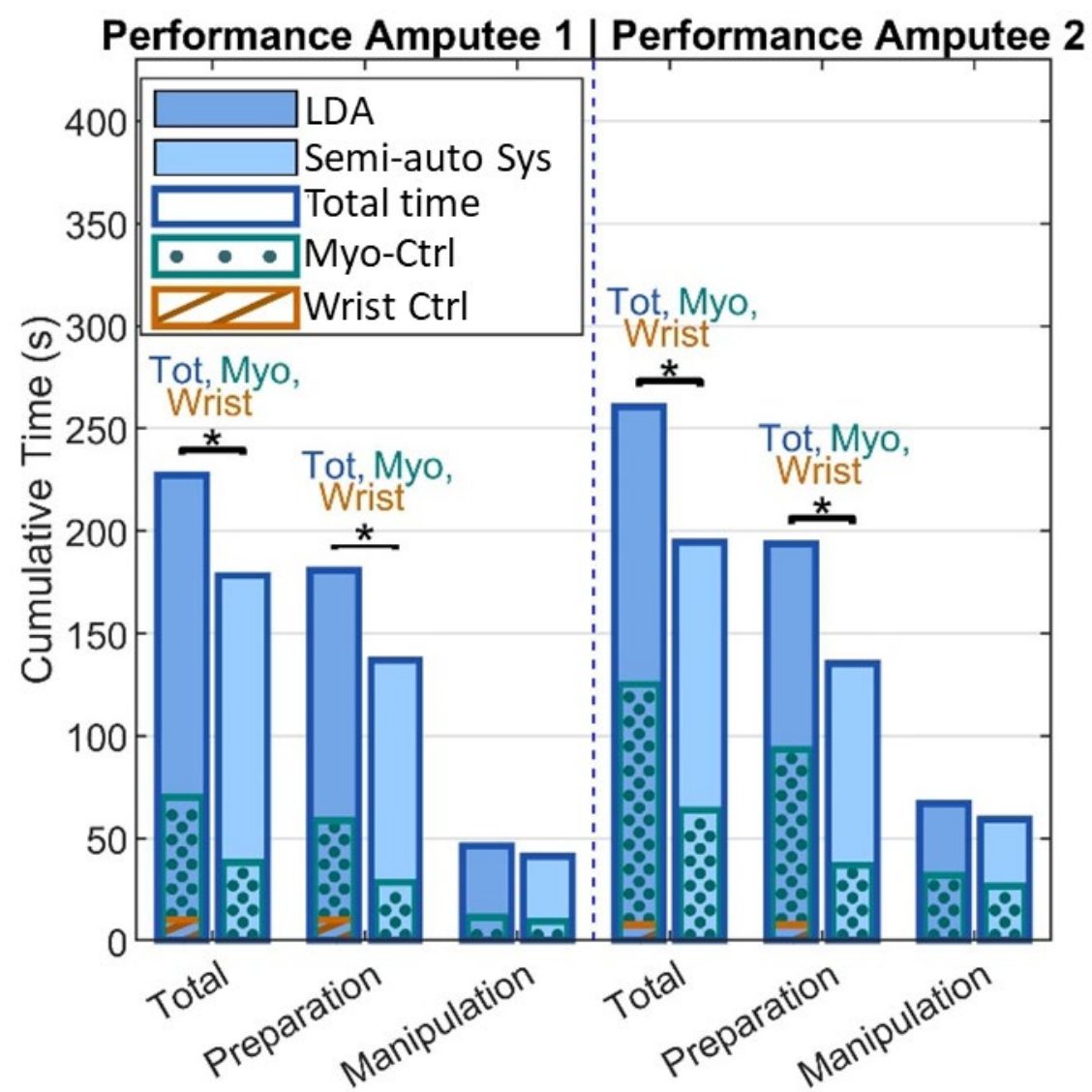

Figure 3.7 Performance of the two amputee subjects during the SHAP tests. The cumulative duration of the test and the total time spent using myo-control are decomposed in the preparation and manipulation phase. The indications above the asterisks stand respectively for a significant difference $(p<0.05)$ in the total amount of time (Tot), the time spent controlling the whole prosthesis manually (Myo), and time spent controlling the wrist manually (Wrist) for each phase of the task. Adapted from [124]

Concerning the amputee group, the first amputee performed significantly faster with the SA system (from 66.8 [9.1] seconds to 57.0 [6.7] seconds), while no significant difference was observed for the total trial time for the second amputee. The first amputee significantly reduced the duration of the preparation phase, while the second one reduced the duration of the manipulation phases significantly. Both decreased the myo-control time during the whole experiment and, more specifically, during the preparation phase. The second amputee also decreased the myo-control time during the manipulation phase.

The SHAP was executed for a total of four repetitions (two control methods $\mathrm{x}$ two subjects). Both subjects successfully performed the tasks in both the SA system and LDA conditions. When using the SA system, both amputees obtained a reduction 
Chapter 3: Assessing the support provided by an autonomous controller when combined with an ML controller

of the total time, the preparation time, the total myo-control time, and the wrist control time for the whole experiment and the preparation phase specifically. No significant difference was observed during the manipulation phase.

\subsection{Conclusion}

These experiments were designed to assess the benefits in terms of time performance of an SA system for hand prosthesis in a realistic environment.

The first experiment reproduced a sequence of tasks constantly affecting the pose of the prosthesis, the position of the objects in the scene, and reducing the possible mental preparation between each manipulation. The results of the able-body participants showed a significant reduction of the total time required to perform the sequence, confirming that the novel man-machine interface significantly improved the time performances compared to the state-of-the-art myo-control. Moreover, the detailed analysis showed that only the preshaping phase of the movement was affected with a significant time reduction as well, which was consistent with the hypothesis that the system only affects this specific phase since it was only active during this phase. The myo-control time and, more specifically, the time used to control the wrist was also significantly decreased, confirming our last hypothesis for the able body participants. Interestingly, the interquartile range of the trial's duration was also significantly reduced, indicating a consistent performance. This uniformity of performance combined with a better average performance lead to the conclusion that the SA system's performance is less sensitive to the user's skill and offers better control to anyone with potentially less time required for the user to train to operate the prosthesis.

The number of amputee participants did not enable a generalisation of the results for the amputee population. Nevertheless, those two case studies showed both the benefits of the novel SA prosthesis. One of the two amputee participants showed the same pattern as the able-body group, significantly reducing the wrist control time during the preshaping phase of the movement. This reduction of the wrist control time 
Chapter 3: Assessing the support provided by an autonomous controller when combined with an ML controller

significantly reduced the myo-control usage time for the same phase. This also decreased the global trial duration. The second amputee showed another behaviour during this experiment. Both movement phases significantly reduced the time used to control the prosthesis and, more specifically, to control the wrist. However, whereas the preshaping phase seemed unaffected by the SA system with no significant time difference observed, the manipulation time was significantly reduced. This behaviour can be explained by the use of compensatory movement to grasp the objects. The instructions given to the subjects were intentionally unspecific regarding the grasp strategy to allow natural grasps and natural behaviours. Therefore, the results suggest that the second amputee used a suboptimal grasp during the pure myo-control trials, enabling him to grasp the object faster at the cost of longer manipulation. The significant reduction of the manipulation phase duration and the myo-control usage duration supported this interpretation. Indeed, the control of the prosthesis was reduced to pure myo-control in the two scenarios. The significant difference could thus only be explained by a difference in the amount of required wrist manipulation caused by a difference of prosthesis pose at the grasp of the object each after the grasps performed using the pure-myo-control. The reduction of myo-control usage during the preshaping phase of the movement confirmed that the participant used the SA system interface to take over the preshaping manipulation during this phase. The SA system interface enabled the participant to preshape the prosthesis in a similar amount of time than his performance during the pure myo-control scenario, leading to better hand position when considering the manipulations to be performed afterwards.

On the other hand, the SHAP results clearly answer the initial hypotheses for both amputee participants. Indeed, the SA system interface condition shows a significant improvement of the time performance, linked to a significant reduction of the preshaping phase duration and the reduction of the myo-control usage and wrist control time. The relatively small manual control of the wrist required by the SHAP, as observed in the results, highlights the benefits of the SA interface.

\subsubsection{Limitations}

As previously mentioned, the amputee population participating in these tests (two amputee individuals) does not draw general conclusions related to the end-users 
Chapter 3: Assessing the support provided by an autonomous controller when combined with an ML controller

application of the semi-autonomous system. The two case studies show an improvement in the total performance in the two experiments. However, the behaviour is inconsistent in the CSI experiment, which shows that further investigations are needed to assert the benefits of semi-autonomous systems for amputee individuals.

The results obtained have been impacted by the limitations of the autonomous controller discussed in the previous chapter, but also by the AR Glasses added to improve the interaction between the user and the autonomous system by limiting the incomprehension of an un-intuitive automatic actuation. The pure myo-control condition was performed without the AR glasses. Those limit the user's field of view, while their weight can lead to higher fatigue. This low wearability of the semiautonomous system might have decreased the benefits it provided. In the future, the AR glasses might be replaced by a newer version with a larger field of view [127], or even when the reduction of the occasional failures and delays makes the system more intuitive. Also, due to the limitations of the autonomous controller, the SHAP could not be used in its entirety. Indeed, some objects were too small or too reflective to be correctly acquired by the artificial exteroception module. This impacts the characteristic of the SHAP of representing the variety of objects and manipulation present in everyday life.

The experiment duration required distributing the participant's training and the measurement of the conditions on different sessions. Because of the temporal variability of the EMG signals, the quality of the myo-control may have differed between the different experiment sessions. The pseudo-randomisation of the sequence of the conditions aimed to handle the effect of this variability.

Eventually, the myo-control proposed to the amputee participants included a co-contraction to switch between the different controllable DoFs. In the standard twochannel control frame, this switch has already been criticised for its potential unintuitiveness and reduced performance when controlling a multi-functional prosthesis [36]. The myo-control implemented for the amputee participants does not enable a direct control for all the DoFs at the same time but answers the low number of distinct muscle contraction patterns that the two amputee participants were able to produce. In a study produced by Li et al. in 2020 [50], a drop of accuracy was observed when 
Chapter 3: Assessing the support provided by an autonomous controller when combined with an ML controller

classifying more than six different muscle contraction patterns performed by amputee subjects. This number is not high enough to directly handle all the DoFs of the prosthesis used in this experiment, which justifies using an alternative design and encourages the development of semi-autonomous systems, reducing the need to control the DoFs of the wrist. 
Chapter 4: Impact of the sharing control modality on the user's performance and workload

\section{Impact of the sharing control modality on the user's performance and workload}

The previous chapter demonstrated the benefits of the SA system compared with the single use of an ML myo-controllers to control a multifunction prosthesis in terms of time performance and the reduction of physical effort of using a SA system to automate part of the preshaping actuation necessary to grasp the intended object.

While the applicability of an SA prosthesis controller to a challenging scenario and its demonstrated potential benefit for the user motivates the development of an autonomous controller, it also raises the issue of the interaction between the user and the autonomous agent. The impact of the shared control modality on the benefits of a SA system should be studied to both integrate it in the design of future systems and understand the user's interaction with such a system.

Many studies in recent years developed autonomous grasping systems and assessed the accuracy with which they reproduced the expected behaviour but did not implement HMI, enabling to merge them with the myo-control of a user (for example 
Chapter 4: Impact of the sharing control modality on the user's performance and workload

[77], [78], [80], [88]). This can be seen as a consequence of the novelty of such systems, which contrasts with robotics [128] or other rehabilitation fields (e.g., wheelchair control [129]) where different SA control modalities are already well established. For the few studies from the upper limb prosthesis field which implemented SA interfaces, several designs of shared control modalities have already been implemented, such as 1) triggered, where the user actively triggers an automatic set of actions computed by the autonomous system with no possible interaction with it [75], [85]; 2) sequential, where there is a strict alternation between the control coming from the user and the autonomous system [101]; or 3) simultaneous, where the two agents control different DoFs at the same time [105]. However, the effect of the sharing modality on the performance and the user's interactions with the device remains unknown.

The study presented in this chapter investigates if different modalities of shared control, in the context of a hand prosthesis user supported by an autonomous controller, impact the time performance, physical and cognitive effort. It was expected that the accuracy of the autonomous controller interacts with the shared control modality used and therefore has a significant impact on the performance. Therefore, the chapter investigates the effect on the performance, the physical and the cognitive workload of different combinations of shared control modalities, and the error level added on the autonomous controller. Standard functional outcomes measurements do not measure all these outcomes. Most of them focus on time performance. To measure the effects on the performance, the physical and the cognitive workload, a new assessment has been developed. The participants performed a dual-task comprising a standard reach-and-grasp task from different objects and from a different side, which enabled them to measure the time performance and the physical workload associated with error level and shared control modality. They also had to perform a reaction task simultaneously by pressing a button in reaction to an auditory tone to measure the cognitive workload on the model of the detection response task [130].

To assess these impacts, an SA system was implemented in a sensor-abundant environment implementing a "Wizard of Oz" concept, a concept already used to study the effect of SA systems in rehabilitation [131]. Thus, while not directly 
Chapter 4: Impact of the sharing control modality on the user's performance and workload

implementable with wearable technology, the SA system developed in this study enabled to measure the interaction between the user and the system accurately.

\subsection{A "Wizard-of-Oz" study design}

In the previous chapter, the system developed with off-the-shelf components provided support to the user in a relatively challenging context. The presented results proved the feasibility of developing wearable SA systems in an object-crowded environment and supporting the user during a continuous sequence of tasks requiring a continuous update of the scene understanding. It proved the benefits of time performance and muscle usage brought by the system in these conditions. However, these systems suffer from the lack of accuracy of the sensors on which they rely. For example, systems relying on computer vision to build an artificial exteroception suffer from factors lowering the performances of RGB-D cameras, such as lighting conditions and the speed of user's movement [132]-[134]. Similarly, myo-control also suffers from factors lowering the accuracy of the muscle contraction pattern recognitions. These non-controllable factors may influence the interaction between the user and the autonomous system.

In order not to be affected by the technical issues such as sensors' accuracy and sensitivity to light or noise, and to focus the study on the shared control modality in a controlled environment, a "Wizard of Oz" [74], [135] study design was implemented. This study design replaces the unreliable sensors with more steady solutions to eliminate or significantly reduce the effect of instability and ensure that the results reflect the effect of the shared control modality and the difference of targeted final prosthesis' pose.

The system developed in this study enabled the two agents (the user and the autonomous controller) to interact with the $3.5 \mathrm{DoF}$ prosthesis previously used to study the effects of the shared control implementation. The shared control module ruled this interaction, and both agents aimed to grasp an object placed in front of the user on the table at a known position. As in the study presented in the previous chapter, the left- 
Chapter 4: Impact of the sharing control modality on the user's performance and workload

hand prosthesis (Michelangelo, Ottobock, Duderstadt Germany) used in this study had 3.5 DoFs, including a two DoFs active wrist with flexion/extension and supination/pronation and two grasp types, palmar and lateral (only the palmar grasp was used here).

The system replicated the SA system developed in the last chapters with some differences. The design of the intention detection implemented in the previous chapters requires the system to wait until the hand reaches the vicinity of the object, some centimetres before the final grasping hand position. While innovative and allowing a prediction of the grasping strategy, this design only enables a relatively late prediction and preshaping of the hand prosthesis by the autonomous controller. The window of interaction between the two control agents is highly reduced. Moreover, this design does not reproduce a natural preshaping timing, which set the draft position of the wrist during the flight of the forearm and potentially adjusts the wrist at the vicinity of the object [136], [137], but suddenly preshapes simultaneously every DoFs of the hand nearby the objects. Methods for predicting the intended final hand position earlier in the movement exist in the literature [84], [97]. These suggest that a stable and accurate prediction of the intention of the grasping strategy is realistic, whether it will be combining the prediction of the final position of the hand in relation to the object or predicting the final orientation of the hand directly. In the "Wizard of Oz" study design implemented, the prediction of the grasping strategy and the object is replaced by given instruction, ensuring that both agents know the final position of the hand. This early "prediction" enables the autonomous controller to reproduce a more natural actuation of the wrist by preshaping the prosthesis during the flight of the forearm.

The commands emitted by the two agents were then integrated by the control fusion module, which implemented the three different shared control modalities, simultaneous, continuous, and sequential.

Figure 4.1 illustrates the SA system implemented in this study. 
Chapter 4: Impact of the sharing control modality on the user's performance and workload

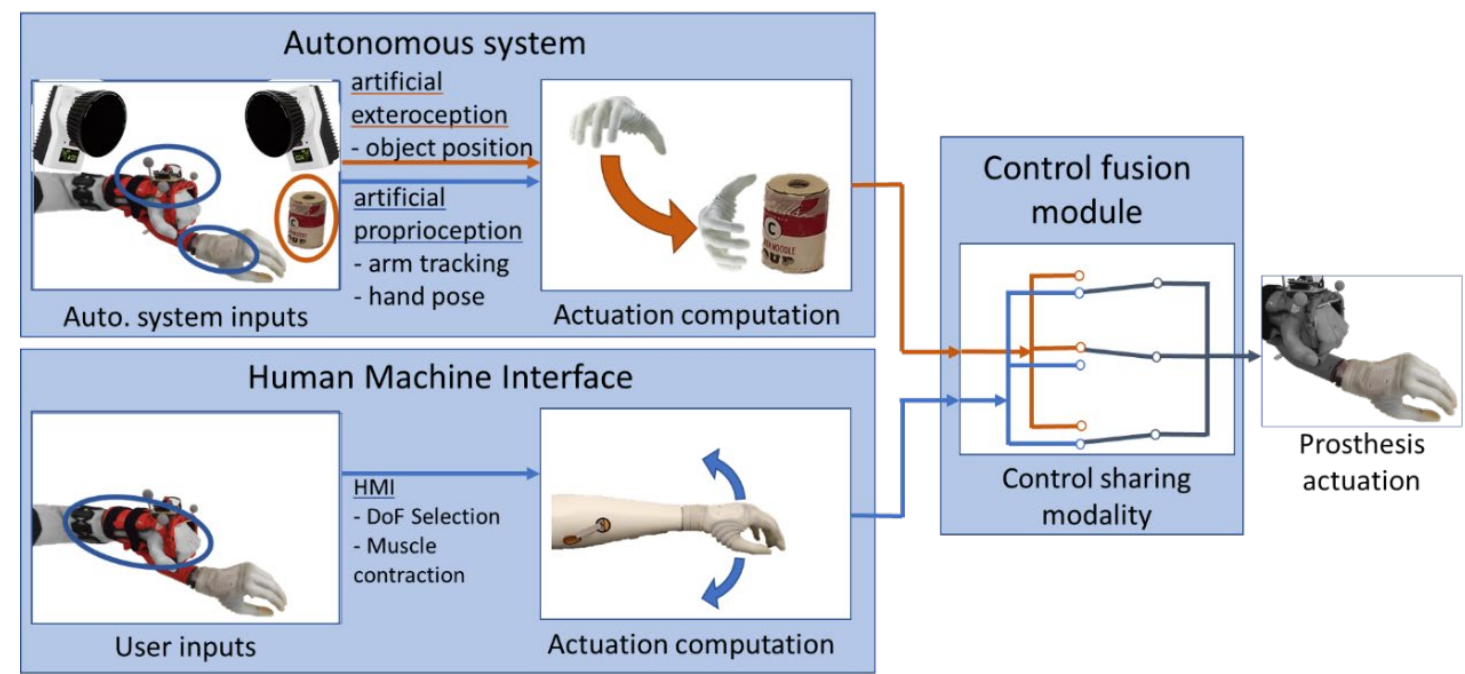

Figure 4.1 Schema of the SA system replicated in a controlled environment. The two controllers, the autonomous system and the myo-controller, compute the prosthesis's actuation based on the inputs they receive. The two outputs are merged in the control fusion module, which builds the command sent to the prosthesis.

\subsubsection{Volitional control module}

This module replicated the HMI provided by an SoA 6 classes classifier by implementing a proportional three DoF prosthesis control associating particular movements of the wrist or the fingers to each DoF of the prosthesis. The SoA approach involves acquiring the EMG via several electrodes placed on the forearm and classifying the muscle contraction pattern. Instead, the system implemented in this study detected the movements of the wrist and the fingers mechanically. Hence, the module comprised two main components illustrated in Figure 4.2. 
Chapter 4: Impact of the sharing control modality on the user's performance and workload

Sensory

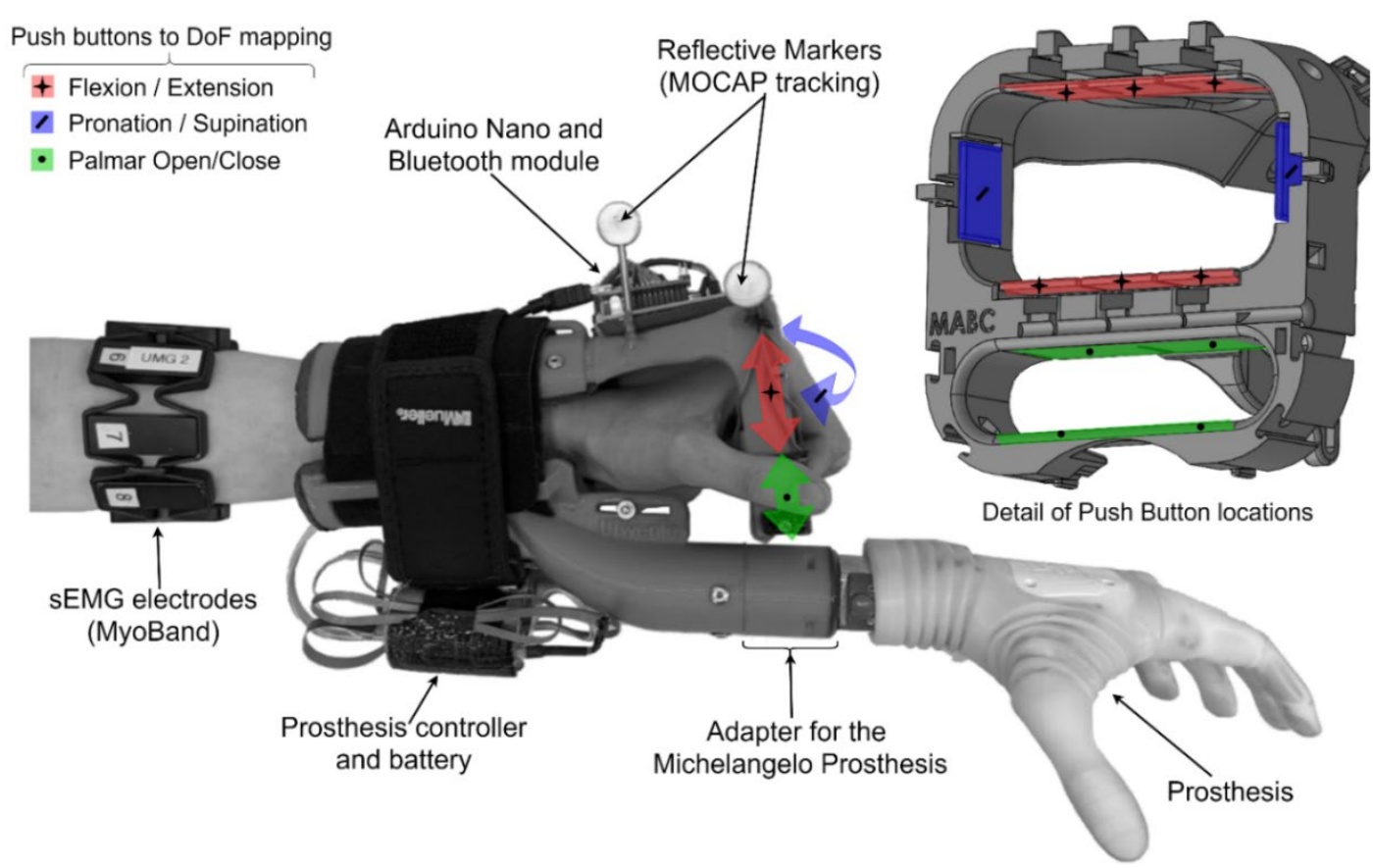

Figure 4.2 Sensorized prosthesis adaptor. The 3D-printed adaptor at the centre of the pictures is fixed to the left forearm of the user and holds the left-hand prosthesis, Michelangelo. Push buttons surround the hand to detect movements of the fingers to associate them to functions of the hand. The armband placed at $5 \mathrm{~cm}$ of the elbow records the intensity of the muscle contraction. The reflective markers constellation fixed on the adaptor enables the mocap system to track it in space.

First, a custom-made mechanical adapter held the hand prosthesis and was attached to the forearm, surrounding the hand of the able-body user. Push buttons were embedded inside, associated with each DoF of the prosthesis. This way, movements of the wrist and the fingers were detected and selected the associated DoF. In case of conflict caused by several buttons pressed, DoFs were given different priorities to ensure that only one prosthesis function could be triggered. The pushbuttons were connected to an Arduino board that streamed their states to the host PC through a Bluetooth connection at $100 \mathrm{~Hz}$. Second, a myoelectric armband containing eight electrodes was placed on the forearm at five centimetres from the elbow. This armband acquired the EMGs of the forearm as an SoA myo-control HMI does. These eight EMG channels streamed the data at $200 \mathrm{~Hz}$ to the host $\mathrm{PC}$ via Bluetooth connection. The data was passed through a Root-Mean-Squared (RMS) filter with a window of $150 \mathrm{~ms}$ and an offset between consecutive time windows of $10 \mathrm{~ms}$. The mean of the eight channels represented the intensity of the contraction of the user, which, once 
Chapter 4: Impact of the sharing control modality on the user's performance and workload

normalised between the intensity of the minimum and $80 \%$ of the maximum voluntary contraction, enabled the user to proportionally control the speed and strength of the actuation of the device.

$$
\begin{aligned}
\text { Speed }_{\text {actuation }} & =\frac{L v l_{\text {contraction }}-m V C}{0.80 * M V C-m V C} \\
L v l_{\text {contraction }} & =\sum_{\text {Channel }=1}^{8} R M S(\text { Channel }) \\
& =\sum_{\text {Channel }=1}^{8} \sqrt{\frac{1}{300} \sum_{\text {sample }=T}^{T+300} E M G(\text { sample })^{2}}
\end{aligned}
$$

In these equations, Speed $d_{\text {actuation }}$ is the velocity command sent to the prosthesis, $m V C$ and $M V C$ are respectively the minimal and the maximal voluntary contraction levels while $L v l_{\text {contraction }}$ is the current contraction level. RMS (Channel) is the value given by the RMS filter, and EMG is the value acquired and streamed by the electrode of the armband. This data filtering induced a delay of around $80 \mathrm{~ms}$, below the critical delay of $100 \mathrm{~ms}$ [138].

\subsubsection{Autonomous module}

The second agent was the ideal autonomous controller designed to preshape the prosthesis during the flight to reach the instructed grasping position once it arrived in the object vicinity. This module received the position and the orientation of the prosthesis tracked via a motion tracking system (Qualisys Ltd., Gothenburg, Sweden) at $100 \mathrm{~Hz}$ and the rotation of the two DoFs of the wrist and fingers via the encoders embedded in the prosthesis. This data constituted the artificial proprioception of the system. The module received the position of the target object as well, which constituted the artificial exteroception of the system.

During each trial, the autonomous system aimed to reach the desired grasping configuration associated with the grasping side instructed to the user. This desired grasping configuration was calibrated according to the user preference at the beginning of each session. The forearm position was tracked and expressed into the 
Chapter 4: Impact of the sharing control modality on the user's performance and workload

percentage of the movement according to equation (4-3) to follow the user's forearm movement and enslave the wrist movement.

$$
\text { Percentage }=\frac{\text { Dist }_{\text {traveled }}}{\text { Dist }_{\text {traveled }}+\text { Dist }_{\text {remaining }}}
$$

In this equation, the Dist traveled is the distance between the hand prosthesis (extracted from the position and the orientation of the forearm) and the rest area, the Dist $_{\text {remaining }}$ is the distance between the hand prosthesis and the object, and Percentage is the percentage of the carried out movement. In case of missing data, the hand prosthesis position was linearly interpolated from the speed and direction of the movement of the last two seconds where the forearm was still tracked.

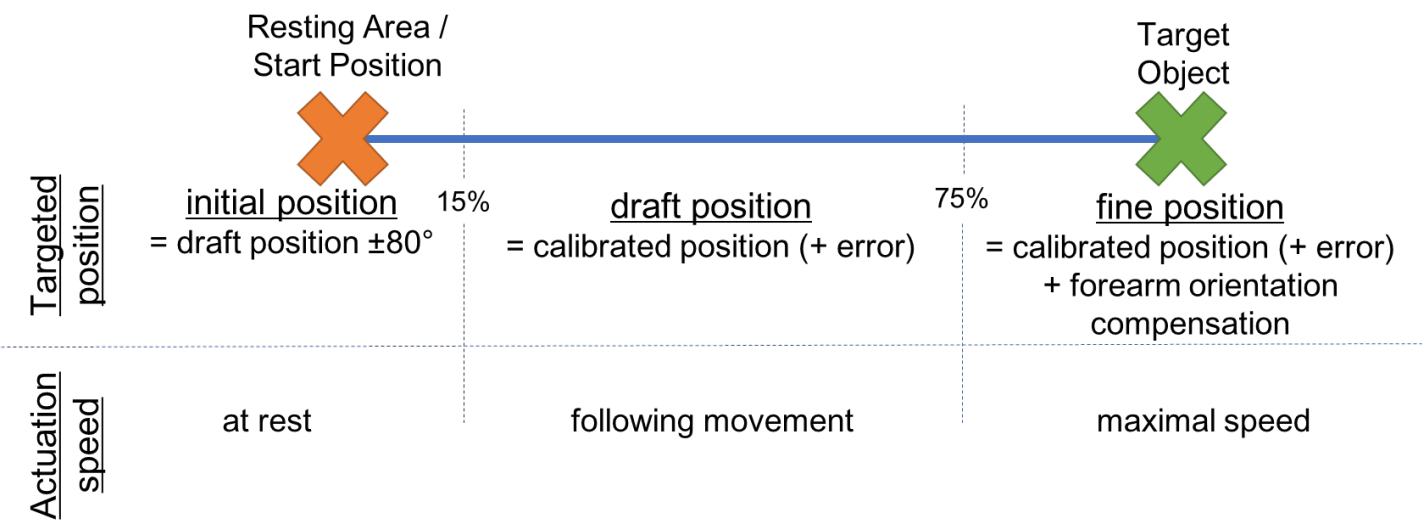

Figure 4.3 Decomposition of the different phases of the automatic preshaping. The phases are related to the position of the forearm

Thanks to this percentage, the movement was split into three phases illustrated in Figure 4.3. In the first one, below $15 \%$ of the movement, the hand remained in starting pose. In the second one, between $15 \%$ and $75 \%$ of the movement, the wrist actuation followed the speed of the forearm to bring the wrist into the final position, calibrated at the start of the session. Hence, the remaining time for the forearm to arrive in the object vicinity (the last $75 \%$ of the trajectory) was computed according to equation (4-4). 
Chapter 4: Impact of the sharing control modality on the user's performance and workload

\section{RemainingTime}

$$
=\frac{\left(0.75 *\left(\text { Dist }_{\text {traveled }}+\text { Dist }_{\text {remaining }}\right)\right)-\text { Dist }_{\text {traveled }}}{\text { Forearm }}
$$

In this equation,Forearm speed $_{\text {is }}$ the average speed computed on the last $200 \mathrm{~ms}$ tracking position data and RemainingTime the time remaining for the hand prosthesis to arrive at the vicinity of the object. Once the remaining time was computed, the DoF actuation speed of the prosthesis was calculated as the ratio between the angular distance between the final and the current position and the remaining time (equation (4-5)). This implementation of the wrist actuation provided the speed curves during the movement similar to the one observed by Dr. Jeannerod during natural prehension movements [136].

$$
\text { Speed }_{\text {DoF }}=\frac{\text { Distance }_{\text {DoF }}}{\text { RemainingTime }}
$$

In this equation, Speed $_{D o F}$ is the computed speed at which the specific DoF of the prosthesis had to be actuated to reach the targeted position when the prosthesis entered the object vicinity. This speed was given in degrees per second before being normalised according to the specifications of the device. Distance $e_{D o F}$ is the distance the DoF still has to travel before arriving at the target position.

In the third and last phase, after $75 \%$ of the movement, the system aimed to make the final adjustment, such as finishing the preshaping if still needed and compensating for a different forearm orientation. The object proximity required to actuate the wrist at the maximum speed authorised by the prosthesis. The compensation for the possible different forearm orientation reproduced the previous system [124]. It also had the advantage of reducing the use of a compensatory forearm rotation from the participant since this movement is not available for many prosthesis users. The hand's orientation was first computed in the world reference frame from the orientation of the forearm obtained from the motion capture system and the position command controlling the wrist of the prosthesis (equation (4-6)).

$$
O_{V_{\text {hand }}}=O_{\text {forearm }} *\left(O_{\text {forearm }} * O_{\text {instruct }_{\text {rot }}} * O_{\text {forearm }}^{-1}\right)
$$


Chapter 4: Impact of the sharing control modality on the user's performance and workload

In this equation $O_{\text {forearm }}$ is the orientation of the forearm of the participant in the world coordinate system and $O_{\text {instruct }_{\text {rot }}}$ is the rotation commanded to the prosthetic wrist. The term $\left(O_{\text {forearm }} * O_{\text {instruct }_{\text {rot }}} * O_{\text {forearm }}^{-1}\right)$ represents, therefore, the expression of the instructed wrist rotation in the world coordinate system. Finally, $O_{V_{\text {hand }}}$ is the orientation in the world coordinate system of a virtual hand prosthesis representing the hand prosthesis after the instructed rotation. Quaternions were used in the model instead of rotation matrices to reduce the quantity of data transferred. The hand's orientation in the world coordinates can then compute the possible compensatory movements (equation (4-7)).

$$
O_{\text {Compensated }}=O_{\text {instruct }} *\left(O_{V_{\text {hand }}} * O_{\text {forearm }} * O_{V_{\text {hand }}}{ }^{-1}\right)
$$

In this equation, $O_{\text {instruct }}$ the rotation and flexion instruction calibrated for the current grasp side. Since the forearm orientation was calibrated to be aligned with the origin when grasping an object, the term $\left(O_{V_{\text {hand }}} * O_{\text {forearm }} * O_{V_{\text {hand }}}{ }^{-1}\right)$ is the expression of the difference of orientation of the forearm with the calibrated position in the virtual hand coordinate system. This way, the difference of orientation of the forearm with the calibrated position was retrieved from the position instruction. Finally, $O_{\text {Compensated }}$ is the system's final orientation of the hand target, compensating for potential over-rotation of the participant's forearm. The final orientation was then expressed in Euler angles to extract the rotation and flexion commands to the control fusion module. 
Chapter 4: Impact of the sharing control modality on the user's performance and workload

\subsection{Implementation of the different shared control $\underline{\text { modalities }}$}

Once the shared control module received the commands emitted by the two agents, it composed the final command to send to the prosthesis according to the shared control modality of the trial. As listed earlier, the three possible modalities of the study were sequential, simultaneous, and continuous, illustrated in Figure 4.4. In the manual-baseline condition, the control fusion module retained any command emitted by the autonomous controller.

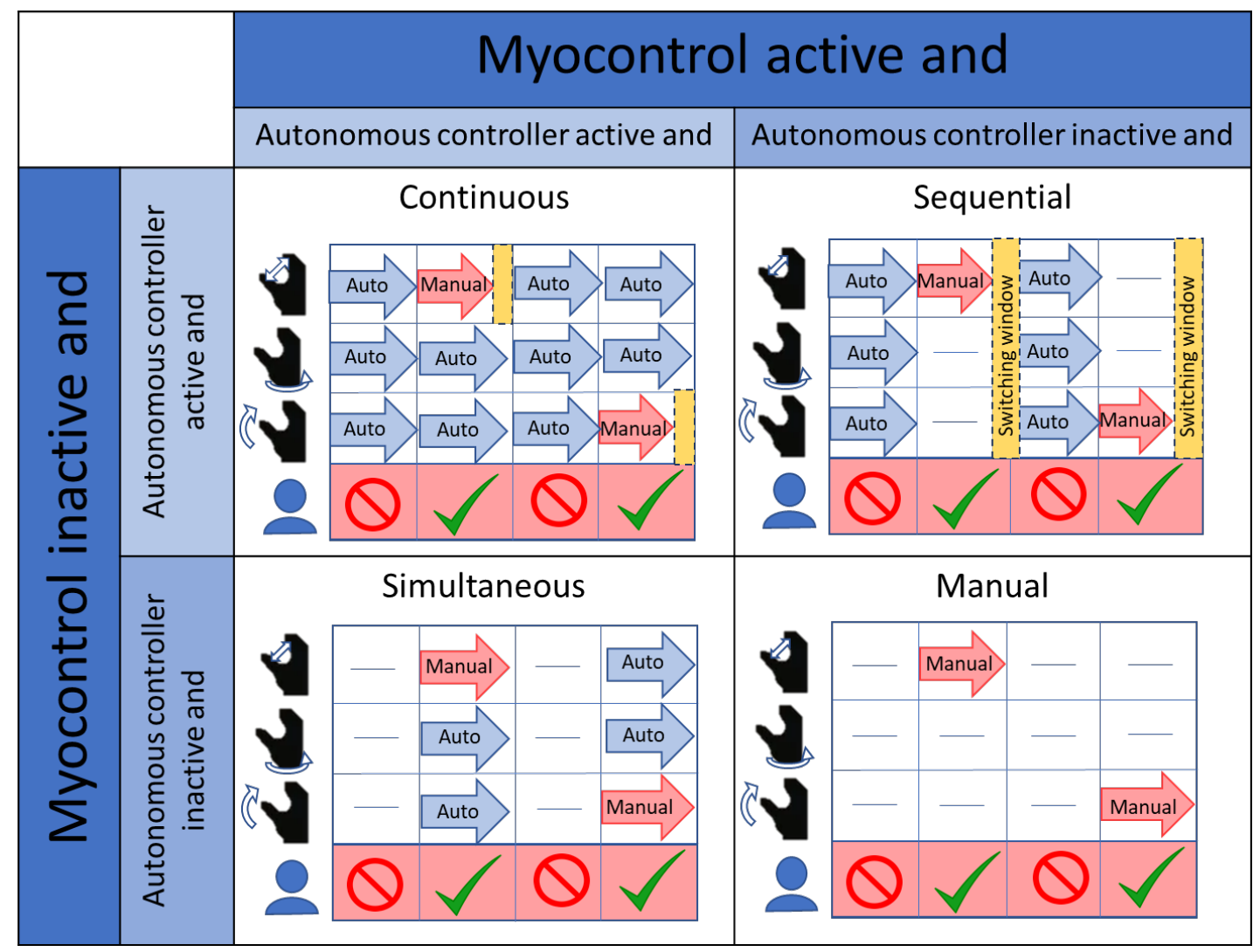

Figure 4.4 Scheme of the different shared control modalities implemented. They are here represented according to their behaviour regarding the myo-control inputs

In the sequential SA modality, the autonomous controller controlled all the DoF of the hand under the condition that the user was not actuating the prosthesis through myo-control. In that case, the commands sent by the autonomous controller were ignored to let the user be the only agent in control. After any myo-control input, a window of 1.5 seconds of inactivity was observed before resuming the autonomous 
Chapter 4: Impact of the sharing control modality on the user's performance and workload

system actuation. This window acted as a temporal filter for the potentially intermittent myo-control.

In the simultaneous SA modality, the autonomous controller was only active during the actuation of one of the DoFs by the user via myo-control. In this case, it actuated the DoF that the user did not currently control. The prosthesis was therefore not actuated when the user did not produce any muscle contraction.

Finally, in the continuous SA modality, the autonomous controller controlled all the DoFs of the prosthesis not presently actuated by the user. A window of 1.5 seconds was observed before handling back to the autonomous controller a previously manually actuated DoFs. In this modality, the autonomous controller was in charge of several DoFs regardless of the user's interaction with the myo-control. 
Chapter 4: Impact of the sharing control modality on the user's performance and workload

\subsection{Experimentation}

The experiments were performed in ten non-disabled subjects (five females and five males), from 24 to 55 years old, all right-handed. The goal of the study and the manual control of the prosthesis and all three SA control modalities were explained to each participant. All subjects signed a written consent form approved by the Ethical Committee of the University Medical Centre Göttingen (22/04/16).

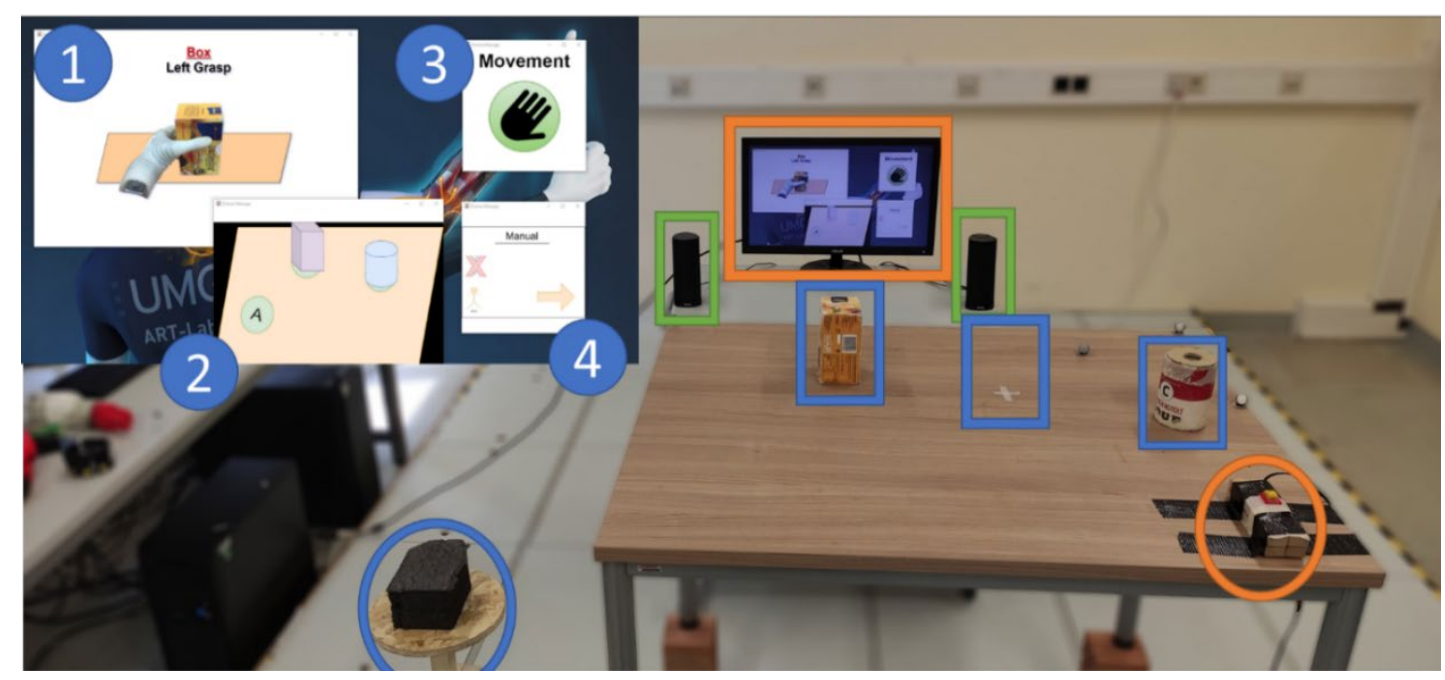

Figure 4.5 Illustration of the experimental setup. The participant started the trial with his forearm on the rest area platform (blue circle). In front of him, on the table, two objects stand in three possible positions (blue rectangles). On the right, a push button (orange circle) enabled the participant to react to the auditory task emitted by the high speakers (green rectangles). The instructions were displayed on the monitor (orange rectangle): 1) the targeted object and grasp side, 2) the position of the objects at the end of the manipulation, 3) the countdown, and 4) the currently implemented shared control modality.

\subsubsection{Experimental setup}

The effect of the three modalities on the three outcomes, time performance, physical and cognitive efforts, have been assessed through a dual-task combining a reach and grasp task and an auditory reaction task. Therefore, the subject was standing in front of a table on which two objects, a box $(130 \times 65 \times 80 \mathrm{~mm})$ and a cylinder ( $117 \mathrm{~mm}$ height, $65 \mathrm{~mm}$ diameter), were standing on two of the three spots marked with tape. The height of the table was adjusted to the height of the participant so that the objects were graspable by the user with a horizontal forearm. On the user's left was placed a platform with foam to place the below part of the adapter between each trial. This platform was called the resting area and marked the starting position of the 
Chapter 4: Impact of the sharing control modality on the user's performance and workload

movement. On the user's right, a push-button enabled the user to react to the auditory task. Finally, two high speakers emitted the auditory cues to which the participant had to react. The instruction and the countdown were visually provided on the screen standing in front of the participant.

\subsubsection{Experimental protocol}

Each experiment session started with the calibration and training of the participant. The participant was first explained the goal and the process of the study as well as the functioning of the myo-control, then equipped with the armband and prosthesis adapter. The maximal and minimal voluntary contractions were acquired by the mean of three high and low muscle contractions for each of the six functions of the prosthesis. These values were then used to normalise the contraction level to control the prosthesis speed of actuation proportionally. The participant was then trained through a series of specific actions of the prosthesis and of object grasps. Once the four different grasp sides had been practised, the preferred grasping position of the wrist and forearm orientation were calibrated for each of them.

Once this was done, the measurement session started. At each trial, the participant was asked to keep the forearm on the rest area platform. The prosthesis was there preshaped in a pseudo-random position. This pseudo-random position was always at $80^{\circ}$ from the expected final position to ensure the same amount of required actuation of the prosthesis for each trial. At the end of the three seconds countdown, the participant was asked to reach the instructed object and grasp it according to the instructed grasp side. The object had to be re-located on the previously free spot, ensuring a relative diversity of object position. The grasp of the object triggered the end of the measurement. This grasp was defined and detected as a none null force exerted by the fingers and none fully closed fingers. Once the re-localisation was done, the participant was required to put back the forearm on the rest area platform and wait for the subsequent trial. Starting at the end of the three countdowns, the auditory task started as well. Auditory signals were emitted and lasted until the reaction of the participant on the push button. After the reaction, a quiet window of either 0.8 or 1.6 seconds was imposed before the next signal. The auditory task stopped with the participant's reaction to the last signal emitted before the grasp of the object. 
Chapter 4: Impact of the sharing control modality on the user's performance and workload

To study the impact of the shared control modalities on the outcomes regarding the error level, three error conditions were implemented: no error, moderate error, and high error. The no error condition does not affect the commands of the autonomous controller. The moderate and large error conditions were added respectively $30^{\circ}$ and $60^{\circ}$ on each wrist DoF with a pseudo-random direction. If the addition of the error to the autonomous controller's decision was outside of the prosthesis' range of motion, then the direction of the error was reversed.

Once the calibrations and training were done, the dual-task was performed. The ten reach-and-grasp conditions comprising the manual baseline and the nine combinations of three shared control modalities and three error conditions were performed through two groups of ten trials. To confirm the validity of the auditory task, a pure auditory task baseline condition was added through four groups of ten trials. The twenty-four groups were then performed in random order. At the start of each group of trials, the shared control modality was communicated to the participant. Fifteen-minute breaks were observed after every 60 trials.

\subsubsection{Outcome measures and data analysis}

The effect of the control-sharing modalities was measured through three outcome measures. The duration of the trial was associated with the time performance. The myo-control duration accounting for the time the participant used myo-control to actuate the prosthesis was associated with the physical effort required to perform the trial. Finally, the delay of reaction, which was the maximal time of the trial needed by the participant to press the push button after the auditory signal, was associated with the cognitive workload caused by using the prosthesis in the specific condition.

Each participant performed 240 trials decomposed into twenty-four groups of ten trials. The two first trials of each group were removed from the analysis to avoid adapting to the new modality on the measures. For each participant, the average values of the outcome variables were computed per condition. None of the outcome variables passed the Kolmogorov-Smirnov test. Consequently, in the case of a significant Friedman test, the differences between conditions were assessed using the Wilcoxon 
Chapter 4: Impact of the sharing control modality on the user's performance and workload

signed-rank test, which considered the dependence between outcomes from the same individual. The obtained $\mathrm{p}$ values were corrected using a Bonferroni-Holm correction.

\section{4. $\underline{\text { Results }}$}

Three outcomes were measured to quantify the impact of the shared control modality, which will be detailed here in sequence.

\subsubsection{Trial Duration}

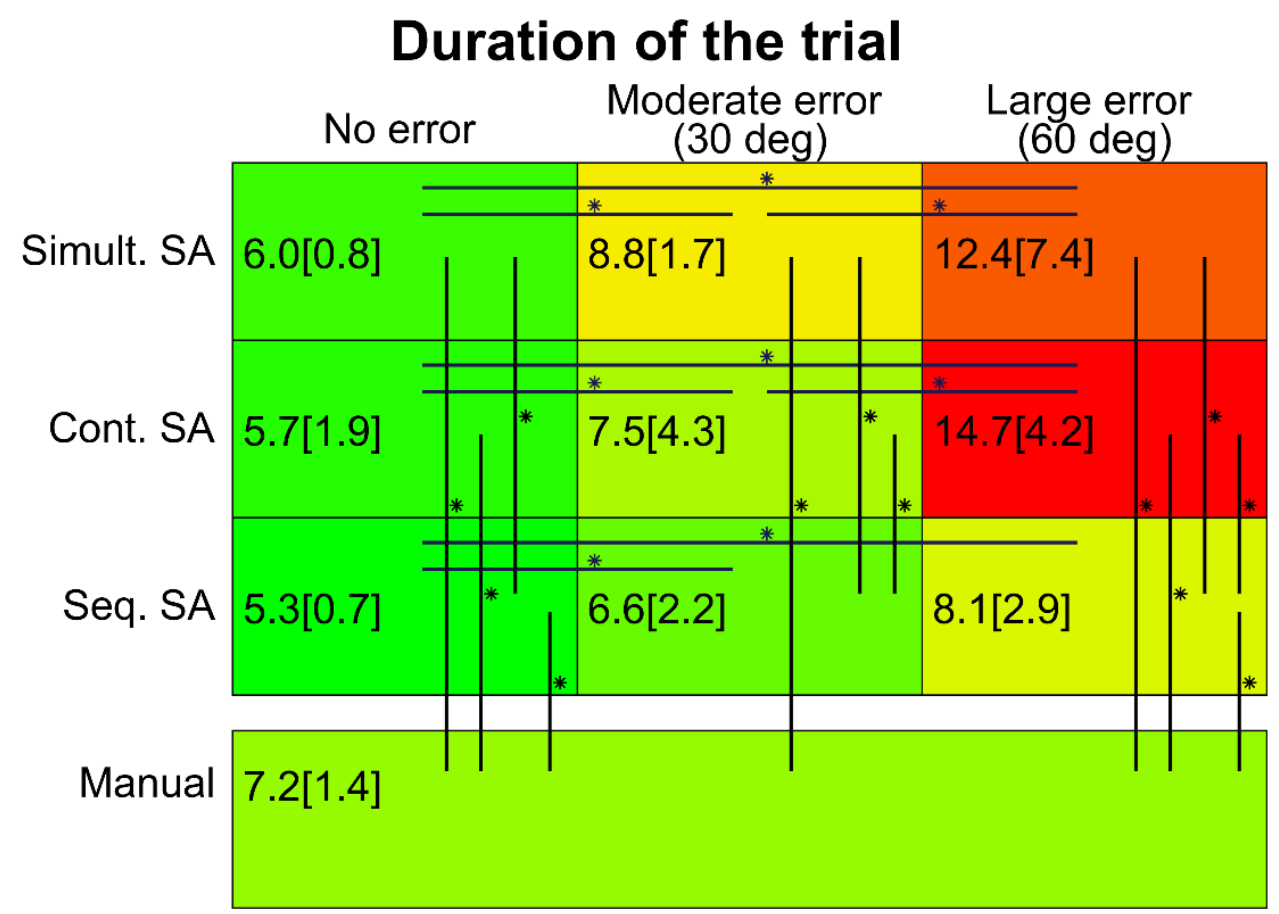

Figure 4.6 Outcomes of the duration of the trial during the different conditions. The depicted values are the medians [interquartile ranges] of the calculated averages for all participants in the respective condition. The colour scale is normalised to the minimal and maximal values of the table. Statistical differences with a p-value lower than 0.05 are marked with a '*'.

As illustrated by Figure 4.6, the increase of error led to the increase of the time necessary to complete the task. Thus, the performance of the three SA modalities decreased, as shown by the significant increases in the trial duration for each modality between the no error condition and the moderate error condition. The second step of 
Chapter 4: Impact of the sharing control modality on the user's performance and workload

added error also increased the trial duration using the simultaneous SA modality and the continuous SA modality. Still, no significant difference was observed for the sequential SA modality.

When errors were added, the decrease in performance shown by the SA modalities also affected the comparison with the manual condition. Compared to the manual condition, all SA modalities significantly reduced the trial duration in the no error condition. In the moderate error condition, the time performance of the continuous and sequential SA modalities was similar to the baseline (no statistical differences observed). In contrast, the simultaneous SA modality led to a significant increase in the trial's duration. During the large error condition, participants performed significantly faster during the manual conditions than the conditions featuring any of the three SA modalities.

Finally, the differences between the trial's duration obtained using the different SA modalities remained similar among the different error conditions. Using the sequential SA modality led to a shorter trial duration compared to the simultaneous SA modality during the three error conditions and compared to the continuous SA modality during the moderate and large error conditions. 
Chapter 4: Impact of the sharing control modality on the user's performance and workload

\subsubsection{Myo-control usage duration}

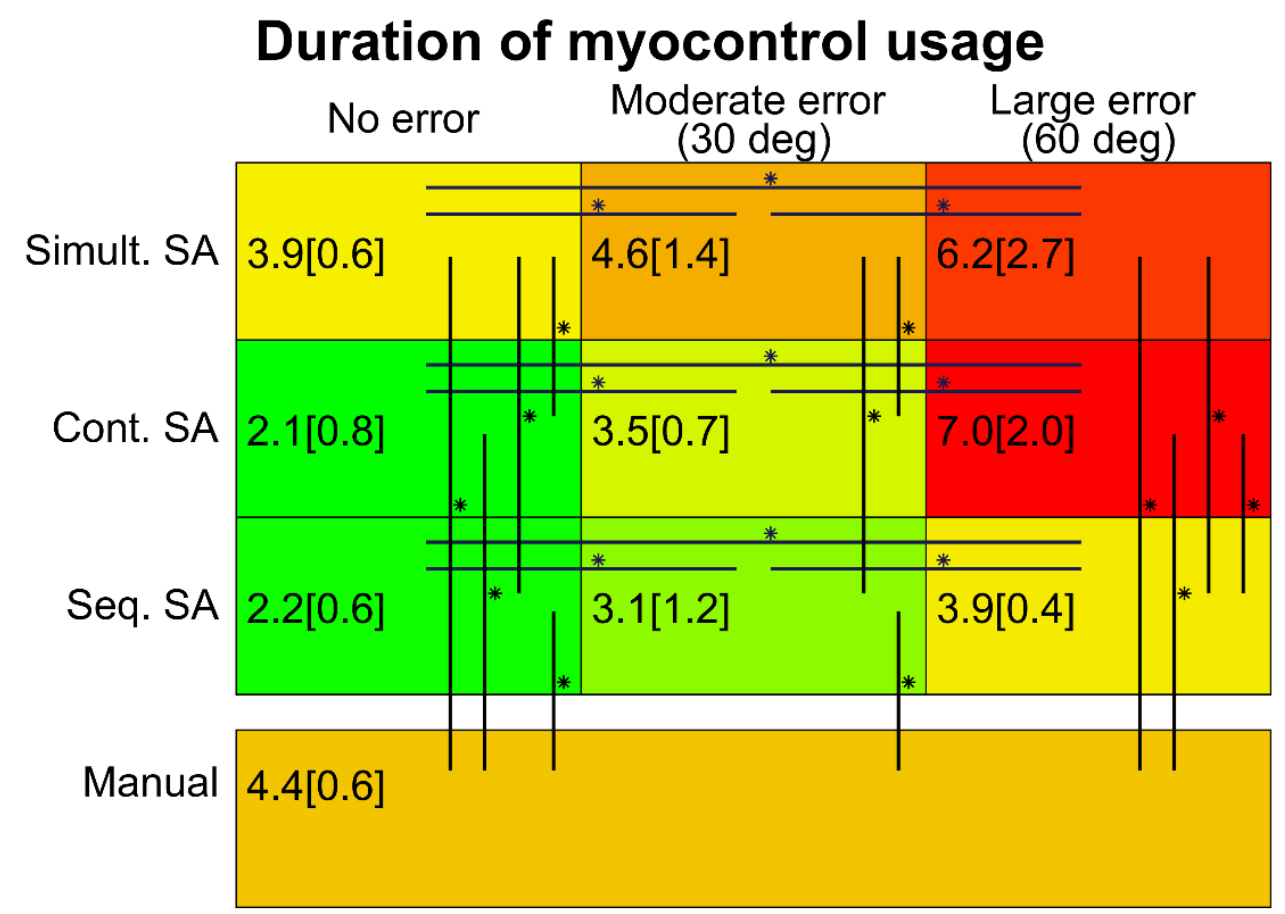

Figure 4.7 Outcomes of the duration of the myocontrol usage during the different conditions. The depicted values are the medians [interquartile ranges] of the calculated averages for all participants in the respective condition. The colour scale is normalised to the minimal and maximal values of the table. Statistical differences with a p-value lower than 0.05 are marked with a '*'.

A similar pattern was observed for the duration of the myo-control usage. Again, the increase of error led to an increase in the usage of myo-control, shown in Figure 4.7. This increase of the myo-control usage's duration was significant for each modality between the no error and the moderate error conditions and between the moderate and the large error conditions.

In a similar pattern to the trial duration, the increase of the myo-control usage duration related to the addition of error affected the comparison with the manual condition. The three SA modalities reduced the myo-control usage compared to the trial performed under the manual condition during the no error condition. Nevertheless, with moderate errors, only the use of the sequential SA modality reduced the myo-control usage duration. In contrast, the continuous and the simultaneous SA modalities led to a similar performance to the manual condition (no statistical differences observed). With large errors, the participants required more 
Chapter 4: Impact of the sharing control modality on the user's performance and workload

myo-control usage when using the simultaneous and continuous SA modalities than pure manual conditions. Only the sequential SA modality remained similar to a manual, with no statistical difference observed.

Finally, the difference between the myo-control duration obtained using the different SA modalities evolved over the different error conditions. Under the no error and the moderate errors conditions, the simultaneous SA modality required significantly more myo-control than the two other SA modalities. Under the large error condition, the simultaneous and continuous SA modalities exhibited similar myo-control duration. Both exhibited a significantly higher myo-control duration than the sequential SA modality. 
Chapter 4: Impact of the sharing control modality on the user's performance and workload

\subsubsection{Reaction delay to the auditory task}

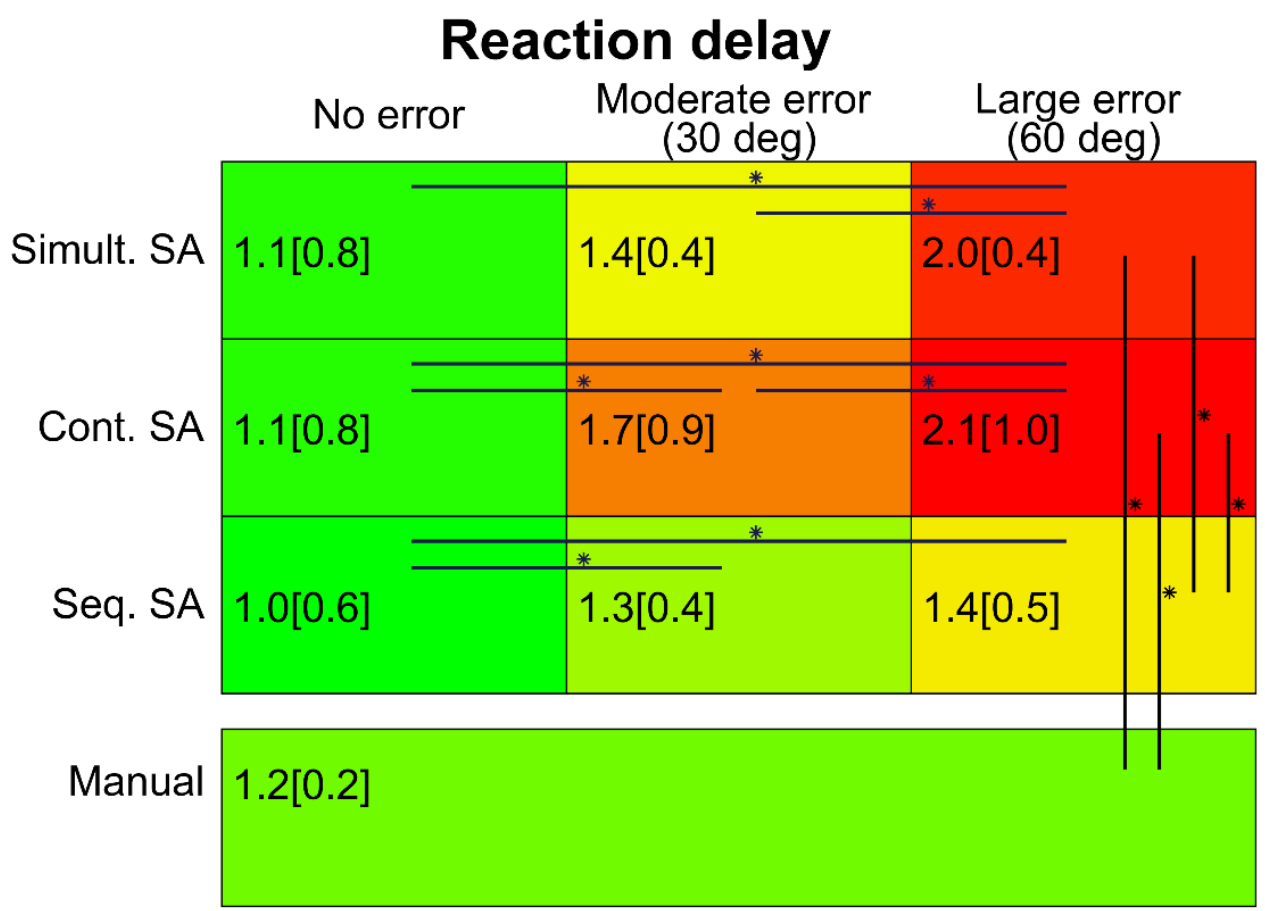

Figure 4.8 Outcomes of the reaction delay during the different conditions. The depicted values are the medians [interquartile ranges] of the calculated averages for all participants in the respective condition. The colour scale is normalised to the minimal and maximal values of the table. Statistical differences with a p-value lower than 0.05 are marked with a '*'.

Illustrated in Figure 4.8, the reaction delay was significantly longer when using the continuous and sequential SA modalities between the no error and moderate error conditions and the continuous and simultaneous SA modalities between moderate and large error conditions. No statistical differences were observed between the manual condition and the three SA modalities under the no error and the moderate error conditions. Under the large error condition, the simultaneous and the continuous SA modalities showed a significantly longer reaction delay than under the pure manual condition. When using the sequential SA modality, participants had faster reactions than when using the continuous SA modality under the moderate error condition and faster reactions than when using either the continuous or the simultaneous SA modalities under the large error condition. 
Chapter 4: Impact of the sharing control modality on the user's performance and workload

\subsection{Representative examples of user's behaviour in continuous, simultaneous, and sequential SA- $\underline{\text { modalities }}$}

During the experiment, different behaviours appeared according to the condition and the user. Some conditions appeared to have similar behaviours, and it is essential to review them in parallel with the median outcomes obtained per condition. They can provide inputs to explain the outcomes of the different conditions.

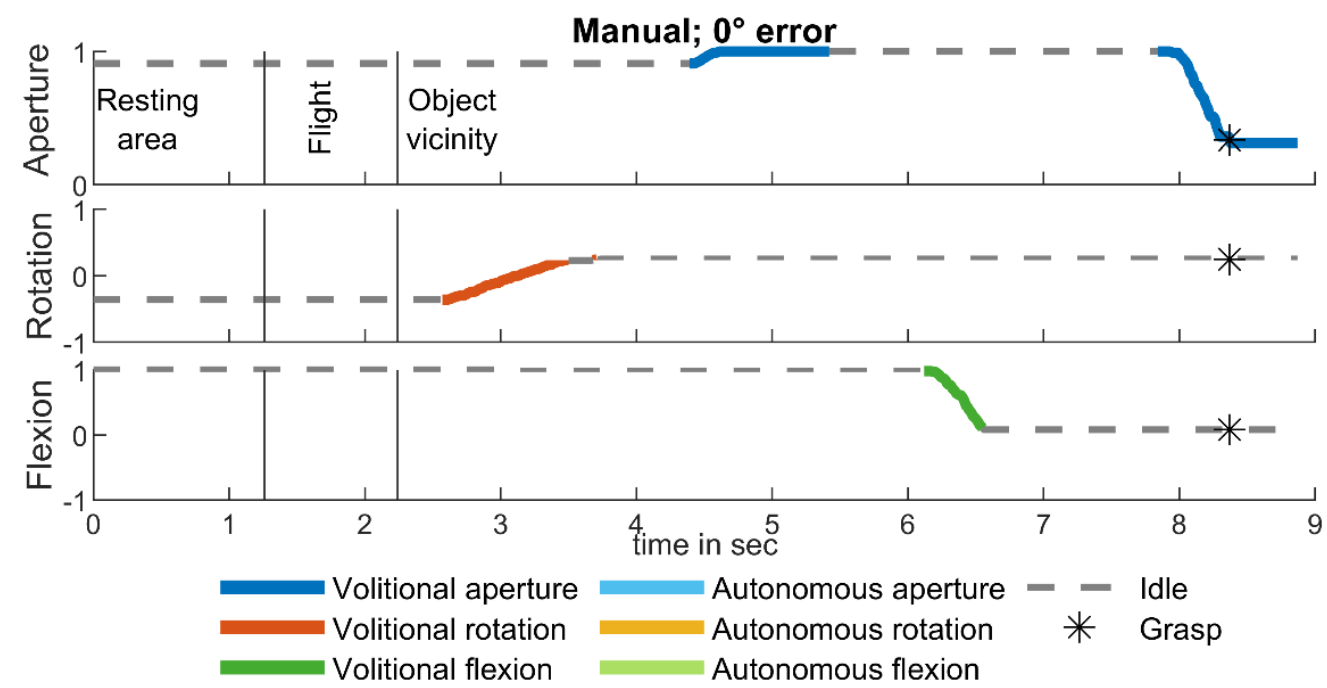

Figure 4.9 Examples of the behaviour of the pose of the three DoFs of the prosthesis under manual condition. The three different DoFs (aperture, rotation, flexion) are represented by one colour each (blue, orange, green), either dark when the movement was due to commands from the volitional controller or light when the autonomous controller was the origin of the actuation. The different phases of the reaching movement are marked by vertical bars: the resting phase (R) from $0 \%$ to $15 \%$ of the trajectory, the flight (F) from $15 \%$ to

$75 \%$ of the trajectory, and the object's vicinity $(\mathrm{V})$ after $75 \%$ of the trajectory.

As illustrated in Figure 4.9, the user had to control every DoFs sequentially to grasp the object from the required side in the manual condition. When the user conditioned the movement (in manual and simultaneous SA modality conditions), the actuation of the prosthesis starts most often in the vicinity of the object. When no error was added to the autonomous controller (Figure 4.10), the continuous and the sequential shared control modalities produced similar pose profiles with the autonomous controller preshaping the prosthesis until a graspable position and the user grasping at the end. The simultaneous shared control condition required the user to be 
Chapter 4: Impact of the sharing control modality on the user's performance and workload

active earlier to preshape the prosthesis. When $30^{\circ}$ of error were added to the autonomous controller (Figure 4.11), a minor correction was often observed to place one of the DoF over- or under-actuated by the autonomous controller. The sequential shared control modality transforms the SA system into a pure myo-control system when the user intervenes (Figure 4.11.C and Figure 4.12.C). On the other hand, the simultaneous and the continuous shared control modalities sometimes led to conflictual situations during the large error conditions (Figure 4.12 A adn B). Indeed, the autonomous controller positioned back a DoF previously corrected by the user while this one corrected a second DoF. This led to cycles of corrections delaying the grasp of the object. 
Chapter 4: Impact of the sharing control modality on the user's performance and workload

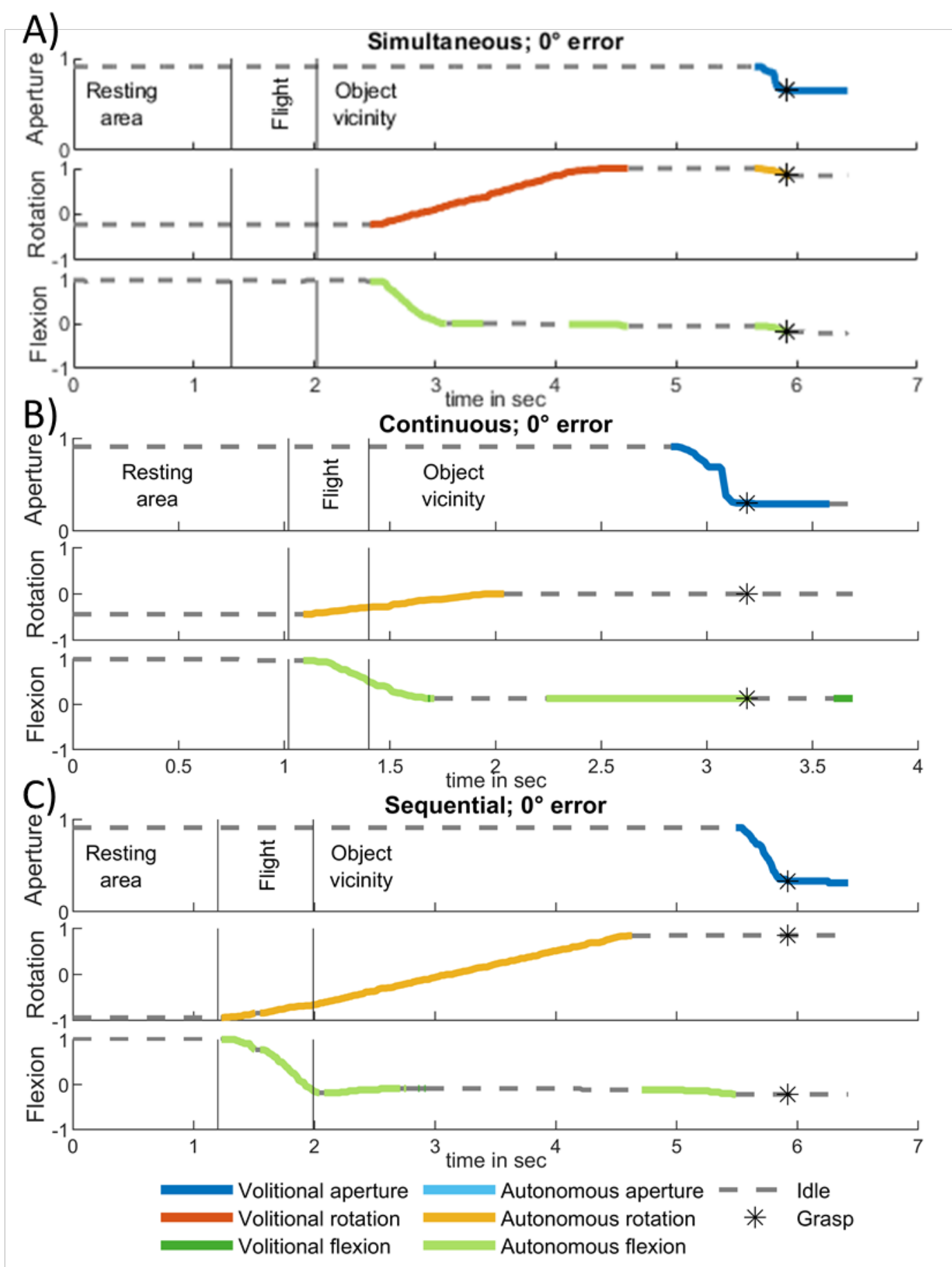

Figure 4.10 Examples of the behaviour of the pose of the three DoFs of the prosthesis under the no error condition for the three shared control modalities. The three different DoFs (aperture, rotation, flexion) are represented by one colour each (blue, orange, green), either dark when the movement was due to commands from the volitional controller or light when the autonomous controller was the origin of the actuation. The different phases of the reaching movement are marked by vertical bars: the resting area from $0 \%$ to $15 \%$ of the trajectory, the flight from $15 \%$ to $75 \%$ of the trajectory, and the object's vicinity after $75 \%$ of the trajectory. 
Chapter 4: Impact of the sharing control modality on the user's performance and workload

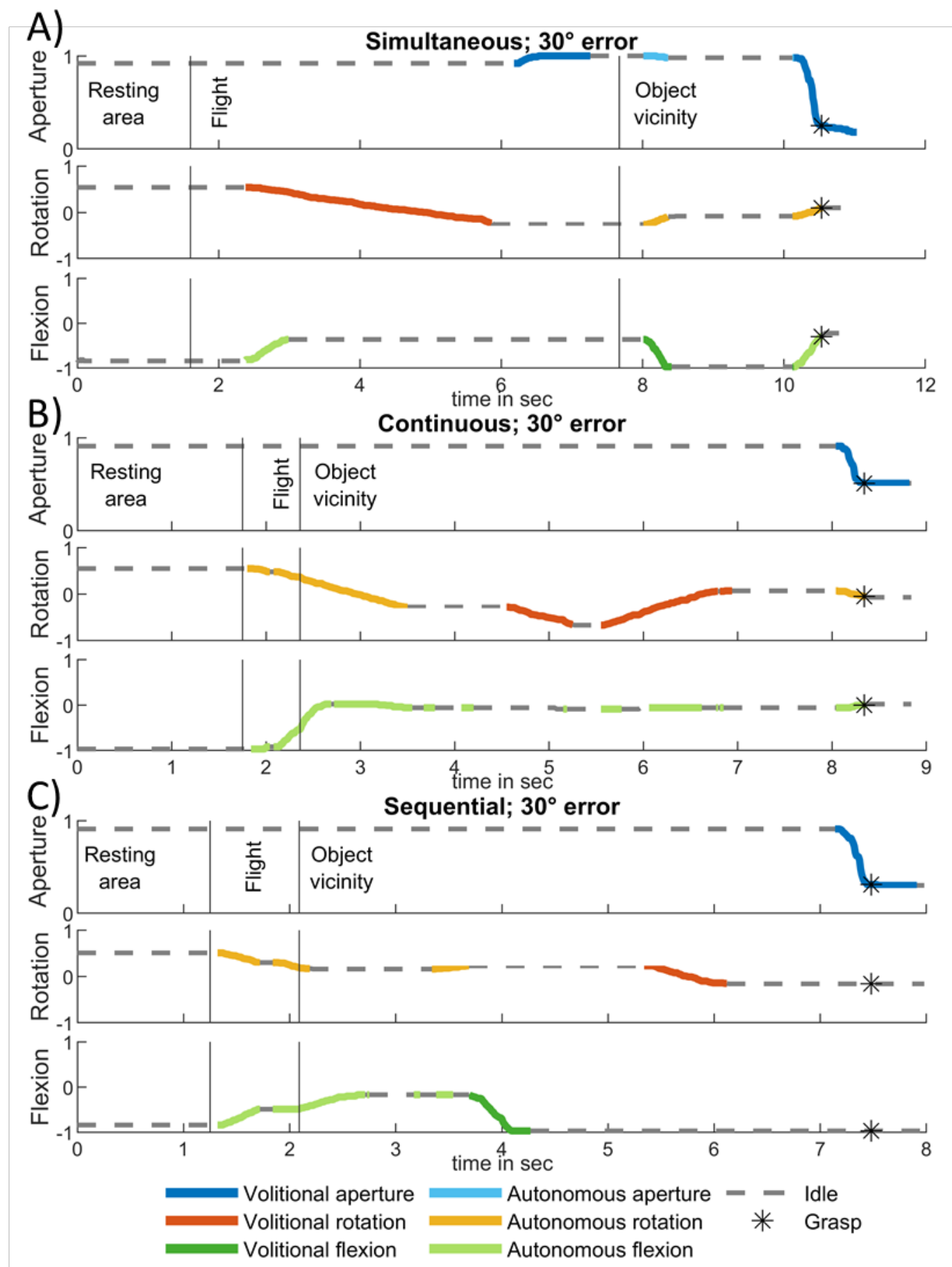

Figure 4.11 Examples of the pose behaviour of the prosthesis's three DoFs under the $30^{\circ}$ error condition for the three shared control modalities. The three different DoFs (aperture, rotation, flexion) are represented by one colour each (blue, orange, green), either dark when the movement was due to commands from the volitional controller or light when the autonomous controller was the origin of the actuation. The different phases of the reaching movement are marked by vertical bars: the resting area from $0 \%$ to $15 \%$ of the trajectory, the flight from $15 \%$ to $75 \%$ of the trajectory, and the object's vicinity after $75 \%$ of the trajectory. 
Chapter 4: Impact of the sharing control modality on the user's performance and workload

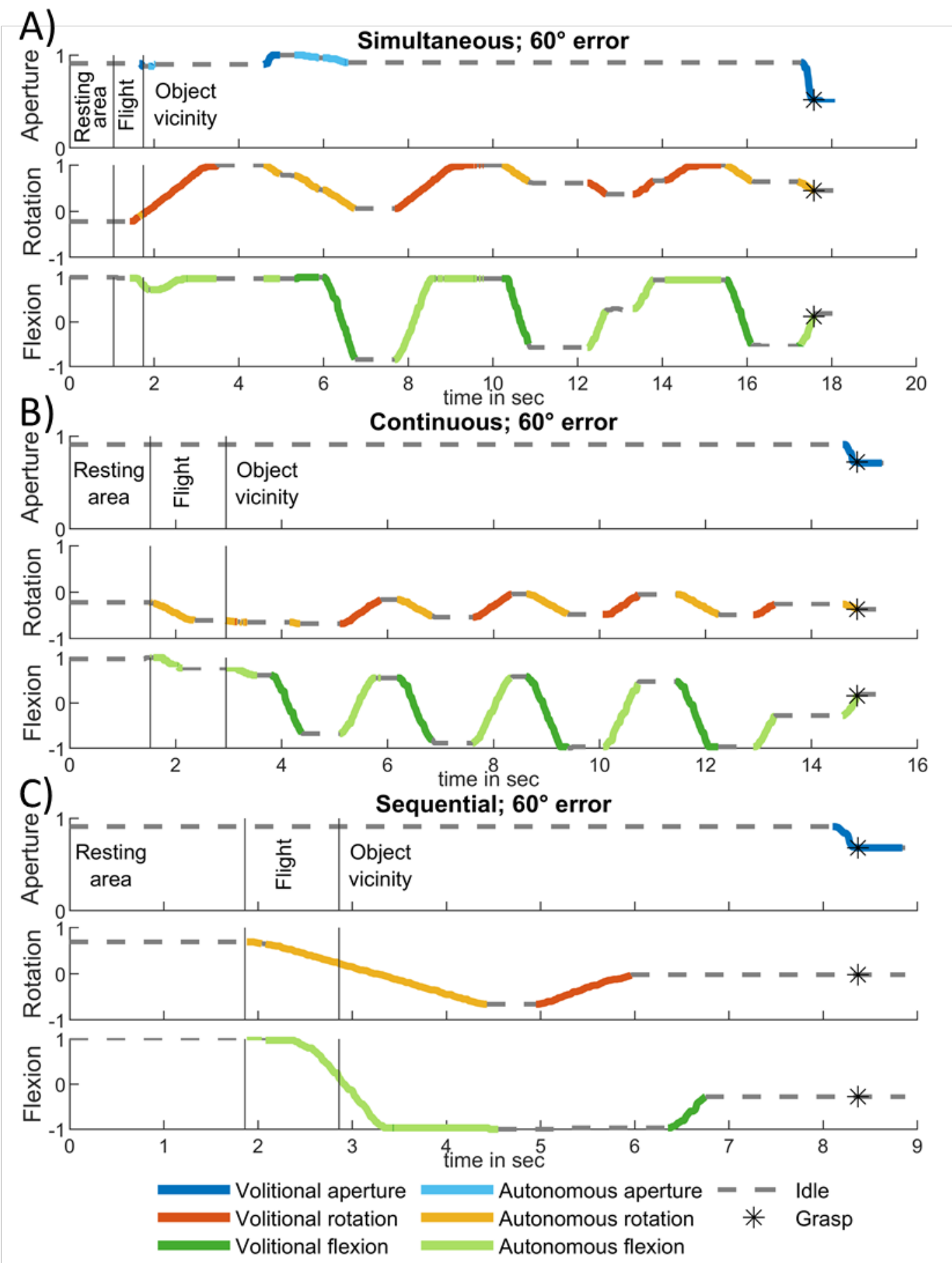

Figure 4.12 Examples of the pose behaviour of the prosthesis's three DoFs under the $60^{\circ}$ error condition for the three shared control modalities. The three different DoFs (aperture, rotation, flexion) are represented by one colour each (blue, orange, green), either dark when the movement was due to commands from the volitional controller or light when the autonomous controller was the origin of the actuation. The different phases of the reaching movement are marked by vertical bars: the resting area from $0 \%$ to $15 \%$ of the trajectory, the flight from $15 \%$ to $75 \%$ of the trajectory, and the object's vicinity after $75 \%$ of the trajectory. 
Chapter 4: Impact of the sharing control modality on the user's performance and workload

\subsection{Summary of the findings}

This chapter aimed to assess the impact of shared control modalities on the performance, the physical, and the cognitive workload. Therefore, ten participants performed the same reach-and-grasp task under different error conditions and different SA modalities.

First of all, it can be noted that the results obtained with the three SA modalities during the no error condition show that the SA supports the user in terms of time performance, which joins conclusions from previous studies [81], [101], as well as the previous chapter. This benefit of SA could be explained by the fact that the SA system actuated several DoFs of the prosthesis simultaneously while the user was limited to one. It is to be expected that the more DoFs are controlled by the SA system, the greater the performance gain. It was also shown that the performance difference was very low between the three shared control modalities when no error was applied. This result may contribute to enable the comparison between different SA systems implementing different shared control modalities. It should also be noted that the simultaneous SA modality required more myo-control usage than the other shared control modalities. This result was also expected since, by design, the SA system was only activated when using myo-control. The significant difference between the sequential and the simultaneous SA modalities shows that activation reduces the benefit of time performance brought by the SA system. The continuous and sequential SA modalities have similar performances, explained by the fact that both share the same advantage of starting the SA support from the beginning of the movement without waiting for the activation from the user. In both cases, the hand was already preshaped at the end of the reaching movement. The user only had to close the hand with no activation from the SA since the hand was already at the final position.

However, as the error increased, the continuous modality moved away from the sequential shared control modality and towards the simultaneous one. These results can be fathered to the simultaneity of the control between the two agents. This simultaneity can lead to a situation of a fight between the two agents where the SA brings back one DoF that the user just corrected. This opposition leads to a sharp decrease of the time performance and increase of the physical and cognitive effort as 
Chapter 4: Impact of the sharing control modality on the user's performance and workload

demonstrated by the significant difference with the sequential modality, which excludes this simultaneity of control. This fighting situation can be dangerous for any system authorising simultaneity, such as [105]. One possibility to avoid this situation is to condition the support of the SA system by a concordance of the actuation of the two agents. If the commands are relatively similar, then the SA is allowed to complete the myo-control from the user [95]

On the other side, the sequential modality seemed to reach a plateau when the error increased. As the results show, the sequential shared control SA modality obtained similar time performance and cognitive workload outcomes between the moderate and large error conditions. One interpretation is that when the user detected the error from the SA system, they switched to manual control by using myo-control and performed the whole preshaping task manually. According to this interpretation, the small significant increase of trial duration for the sequential shared control modality under the large error condition compared to manual baseline would be due to the time for the participant to realise the error from the SA system. Although requiring greater cognitive effort than without error, the sequential shared control modality in the large error condition still shows a similar reaction delay to the auditory task than the manual baseline.

Overall, in the setup implemented here, the sequential modality seems to stand above the other SA modalities because it does not require an action from the user to activate it, reducing the physical effort required and increasing the time performance. It also allows the user to have complete control over the prosthesis if errors appear in the system. This modality has already been used in at least one recent SA system [101].

The sequential modality is similar to a triggered system since those conditions the activation of the autonomous controller by an action or a signal emitted by the user and do not allow simultaneous control from the two agents. Trigger systems use, for example, muscle contraction[75], [82], or EEG [85] to condition the support provided by the autonomous controller. In this perspective, the sequential modality conditions the activation of the autonomous controller by the absence of a user signal, which does not require a specific action from the user. This constitutes, therefore, a transparent trigger condition. 
Chapter 4: Impact of the sharing control modality on the user's performance and workload

\subsubsection{Limitations}

The "Wizard of Oz" study design enabled limiting the effect of the inaccuracy of sensors or the myo-control in the interaction between the user and the autonomous controller. It enabled to study more precisely the effect of the difference of target prosthesis' pose when the autonomous controller and the user collaborate to preshape the prosthesis. Each technological improvement makes this setup more realistic. However, the lower accuracy and robustness of the SoA myo-control and autonomous system does not allow the conclusion of this chapter to be directly transposed to current semi-autonomous configurations. As a matter of fact, the volitional control implemented here impacted the effort required to actuate the prosthesis and the confidence the participant could place in their control. This may impact the incentive for the user to rely on one controller or the other.

The results presented in this chapter exclude amputee participants. The experience that the prosthesis end-users gather in their everyday life on the control of their prosthesis may influence the impacts of the shared control modality on the three outcomes collected. The choice of the shared control modality may also be made considering other outcomes such as the impact on the embodiment of the prosthesis. Indeed, the embodiment is an essential factor for the acceptance of the prosthesis, and the addition of a second agent controlling the hand may interfere with the feeling of ownership and agency that the prosthesis' user usually has with his limb's replacement device. It might be necessary to condition the actuation of the hand with a muscle contraction to ensure the feeling of agency. In the study [139], the authors Tsakiris and Haggard claim that the movement does not have to be congruent with the intent of the individual to produce a feeling of agency but needs to be simultaneous. This would then justify the use of a simultaneous shared control modality.

Finally, the experiment proposes a short task with a few seconds of preparation during which the participant can plan the actuation necessary to complete the task. This may influence the impact of the SA system on the performances. While forearm movement remains natural because it is not affected by the limb deficiency condition and therefore can be good support for intention detection, the exact actuation of the wrist may require some cognitive workload. The difference in cognitive workload can 
Chapter 4: Impact of the sharing control modality on the user's performance and workload

be more significant if the user does not have the time to plan the actuation before. This would require either a sequence of movements like in the previous chapter or to show the initial prosthesis pose and the instructions to the user only at the start of the trial. 


\section{Discussion of the dissertation}

At the beginning of this thesis, the prosthetic devices state and the current myocontrol interfaces were exposed. The gap between the increasing number of actionable prostheses DoFs and the limited bandwidth of the myo-control commands was presented to conclude that the myo-control bottleneck prevents the full use of dexterous prostheses and their active wrist. The two solutions currently developed (pattern recognition algorithms and multi-modalities controllers) were described. Since these solutions have been developed separately, a substantial lack of knowledge on the impact of their combination was identified. Therefore, in the further progress of this thesis, two studies were dedicated to investigating this matter by the mean of newly developed semi-autonomous systems. 


\subsection{Summary of the findings}

The first chapter proposed a method to preshape the prosthesis based on its position in the scene. The goal was to study the applicability of an autonomous controller in a dynamically changing, object-crowded scene. Hence, an autonomous controller was developed, comprising several innovations such as the whole scene modelling and the preshaping of the hand based on its position. Its functioning is based on the fusion of several modalities, including computer vision, inertial measurement units, pressure sensors, position encoders, while providing feedback to the user through augmented reality glasses. This diversity of sensors enables the system to perform a full scene analysis using a SLAM algorithm. In contrast to other studies, which associate a single grasp strategy to the recognised or analysed object [75], [77], [78], [80], [88], [98], [108], the developed SA system adapts the grasp strategy to the intention of the user, through real-time tracking of the forearm and of the surrounding, and a continuous update of the preshape fitting to the context. This continuous support provides the preshape of a two DoFs active wrist, while most autonomous controllers developed until now only preshape one wrist DoF, if any.

The second chapter aimed to determine if using an SA controller combining a semi-autonomous controller with an established state-of-the-art machine learning myo-control could provide better performance than using this myo-control alone. An SA interface (the SA system) was designed by associating the autonomous controller developed in the previous chapter with an LDA myo-controller. This collaboration between the two controllers was ruled through a sequential shared control (traded control) and supported by visual feedback through an augmented reality display. This SA system has been assessed in clinical tests where no other SA systems have been tested before to confirm the range of application of the autonomous controller developed previously. It performed thus, in a standard test known for reproducing ADLs, in a scene where objects were numerous and touching each other, which challenges the computer vision and the intention detection programs, and were relocated. Eight able-bodied and two amputee subjects participated in this study. A detailed analysis enabled to demonstrate 1) the increase of performance compared to the SoA ML controller alone due to faster preshaping, potentially partly explained by 
the simultaneity of the movements performed by the autonomous controller, 2) a reduction of the physical effort required to perform the task since the muscle contraction was not anymore necessary to preshape the prosthesis, 3) a reduction of the wrist control, leading to a reduction of the number of different actuation required to perform the task which is a step in the direction of a simplified control, 4) a reduction of the variance of the performance, bringing the users to a similar level less impacted by their original skills. These results motivate the development of active wrists and more advanced SA systems, which could enlarge the benefit observed in this study to more detailed object interaction or through a system more comfortable to wear for the user.

Finally, the third chapter focused on the impact of the design of the shared control modality of SA controllers for prosthetic hands. Hence, a review was done on the shared control modalities implemented in the different SA systems from the literature. This review concluded by establishing a lack of knowledge regarding the impact of the shared control implementation, both about its effect on the performance and the overall user interaction with the prosthesis, and the lack of consideration of these effects when assessing the developed SA interface. To contribute to answering to this lack of knowledge, the detailed effects of three different shared control modalities on the time performance, the physical and cognitive efforts have been assessed. The interaction with the accuracy of the autonomous controller has also been investigated through the addition of errors on the autonomous controller decisions. Therefore, an SA system has been implemented in a controlled environment, reproducing a realistic myo-control and realistic hand tracking with almost flawless accuracy. The controlled environment enabled 1) to implement a system capable of preshaping the prosthesis in flight, 2) to implement an errorless intention detection, and 3) to accurately control the error added on the decision of the SA controller. The data of ten participants, who performed a dual-task combining a reach-and-grasp task with an auditory reaction task, has been recorded. The results show that the time performance and the physical workload are impacted by the shared control modality implemented. This impact changes and increases when the level of added error increases. The benefits of adding a SA system over a pattern recognition system were impacted as well. The design of the sequential SA modality, assimilable to a traded 
control, seemed more advantageous than the two other modalities thanks to its flexibility and its performance. It offered the support of the autonomous controller when this one made sense and limited its impact when the support was not possible nor desired. These results stressed the importance of implementing and the clinical tests when studying SA systems since the same implementation obtained different outcomes according to the chosen shared control modality. It also illustrated the risk of fixing one grasp strategy per object, leading to conflicts between an autonomous controller with a single grasp solution and a user who naturally has several grasp solutions.

\subsection{Implications of the findings}

Overall, this dissertation has shown that combining an autonomous controller to an ML myo-control can improve the performance when performing a reach and grasp task by increasing the time performance and reducing the required physical effort. It also shows that these results apply to a more extensive range of scenarios than previously tested, such as an object-crowded scene or a standard rehabilitation test with complex object shapes. The possible interactions also evolved by enabling a sequence of manipulation without re-initialising the system and continuous support in synergy with the user, without requiring extra steps in the prehensile process. It also demonstrated that performance and physical workload benefits partly depend on implementing the shared control modality ruling the interaction between the user and the autonomous controller.

The performance improvement brought by the addition of autonomy in the frame of an advanced pattern recognition myocontrol extends previous studies. Indeed, the increase of performance brought by the addition of autonomy has been previously demonstrated in the frame of the historical and most basic two-channel control, during a simple manipulation (reach and grasp task of a single object) [81], [108], or during more complex bi-manual manipulation with an upgraded two-channel control with control of the wrist rotation through elevation of the shoulder [101]. However, improved myo-controllers use pattern recognition algorithms to answer the 
task performance and the amount of muscle contraction needed to actuate the prosthesis manually. The better performances obtained when associating an autonomous controller shows that there is still a marge for improvement of the manual control before reaching the mechanical limitation of the device and that this marge can be partially covered through the automation of the hand. The significant improvements in the total duration of the test performed by the participant also show that systems focusing on the preshaping of the hand can still significantly improve the global performances. It might be interesting to examine the daily use of the hands to search for other sub-task repetitive and frequent enough to bring significant improvements through automation. Eventually, the gain of performances obtained by a semiautonomous system relying on wearable sensors in a complex setup shows that the technology is mature enough to propose a semi-autonomous system performing in real-life conditions.

The benefits brought by the pattern recognitions myocontrol providing direct control over several DoFs did not seem sufficient to answer the complexity of the control of a multi-function prosthesis since this complexity still encouraged the development of under-actuated mechanical solutions. These under-actuated solutions deprave the dexterity of the prosthesis to simplify the control of the device for the user drastically. The significant reduction of the wrist control during the manipulation obtained during this dissertation compared to the advanced myocontrol alone tends to indicate a simplification of the control while preserving the diversity of functionality offered by the medical device. The reduction of the variability across subjects when using the SA system also shows a reduced learning time since the autonomy provided by the system covers the potential lack of skills from the user. Overall, SA systems should not only be considered as a solution to simplify the interaction between the user and its prosthesis anymore but as a complement of the most advanced myocontrol to simultaneously facilitate the full use of the prosthesis by the user and increase the task performances in everyday life conditions.

In addition to the justification of the further development of SA systems, this dissertation brought precise elements to improve the design of future SA systems. While different implementations of shared control modalities are already proposed to include the developed autonomous controllers into SA systems such as sequential, 
simultaneous, or continuous, this dissertation investigated their effects to understand better the impacts of some design components of this element of any SA interface. Thus, the effect of these modalities on the performances, cognitive workload and muscle usage has been studied, demonstrating their similarity at the best level of accuracy of the autonomous controller but highlighting the danger of conflicts between the user and its own hand replacement when the accuracy of the autonomous controller degrades. By highlighting the impacts of the control sharing modality on the performances, future studies are encouraged to pay particular attention when designing and describing the SA systems but also when comparing them to the systems previously developed ones. Finally, the results obtained encourage using a traded control modality design for upper limb rehabilitation devices to disable involuntary movements of the limb replacement and warns for the risk of associating single grasping solutions to the object of interest, potentially leading to conflicts.

\subsection{Future Development}

The results obtained in this dissertation demonstrated that SA systems can already benefit the user. On this basis, it seems promising to improve SA systems based on a more substantial inclusion of interdisciplinary findings. For example, studies providing a better understanding of human grasps such as joint synergies [99], [140], finger synergies [141], object-oriented grasps [142], [143] may be used to benefit the prosthesis control through SA systems. Instead of leading to conflicts, unsure intention prediction could therefore lead to a preshape of the prosthesis based on other information such as the most common finger pose when grasping this type of object or a base position of the fingers and the palm leading to the shortest path for all the probable grasp strategies associated to a given object.

Additionally, the benefit of using an autonomous controller with an ML myocontroller does not reduce the interest in improving advanced myo-control. As a matter of fact, advanced pattern recognition algorithms may give more inputs for the SA system to predict the user's intention. When based on the same sensors on which the SA system is relying, such as IMUs, sensor fusion can improve the accuracy and the 
robustness of the myo-control [64]-[66]. Based on the same sensors, synergy could be implemented between the two controls. In such a synergy, the lack of accuracy and robustness of the myo-control could be detected and corrected by the solution provided by the autonomous controller when high uncertainty is detected. On the other hand, the output of the myocontrol could be integrated into the intention prediction to provide a better understanding of the user's plans and better support them.

SA systems provide the automatic actuation of several DoFs of the prosthesis and therefore aim to reduce the physical and cognitive workload associated with the task. As demonstrated in this dissertation, this can have immediate benefits for the user. However, it adds a second agent which controls the device.

The first goal of prosthetic devices is to replace a missing limb, which heavily impacts the relationship between the patient and the prosthesis. This enables, for example, the treatment of phantom limb pain through the presence or the use of the prosthesis [144]. The addition of a second agent may induce an issue for the user's acceptance and embodiment of the prosthesis.

The sense of embodiment is the recognition of something as part of the biological body and can be broken down into several components [144]: ownership, location, and agency. Probably less impacted in the case of the addition of a SA system, the sense of self-location corresponds to a determined volume of space where the user feels situated [145]. The sense of agency is the sensation of global motor control, including the subjective experience of action, control, intention motor selection, and the conscious experience of will [146]. Eventually, the sense of ownership refers to the self-attribution of a body [147]. Upper-limb prostheses do not provide feedback usually, and therefore the user does not have a proprioceptive input from the movement that the limb replacement device produces. This can perturbate the feeling of ownership of the user for its prosthesis and justify several studies to tackle this issue [148]-[150]. It seems, however, that this lack of direct proprioceptive feedback and the failure of the usual rubber hand experiment usually used to assess the sense of ownership do not prevent the embodiment of the prosthesis by the user [151]. On the other hand, the feeling of agency was until now preserved since the different myocontrols, historical or more advanced, enable direct control over the 
prosthesis joints. By producing movement of the prosthesis not explicitly intended by the user, these movements may interfere with the sense of agency. It is already known that the movement's intention does not need to be congruent with the final actuation of the limb [152], but the temporality of the movement plays a significant role [153].

Considering its role in the acceptance of the prosthesis by the patient and its importance to alleviate phantom limb pain, embodiment could be considered one of the key metrics when assessing prosthesis controllers. While standard myo-control is limited in bandwidth and can be frustrating, SA systems can actuate every joint of the prosthesis simultaneously, which reproduce the natural movement of a hand. Therefore, it might be essential to study the other conditions under which a SA system can still participate in the embodiment of the device by the user to provide them back the feeling of having a natural hand. 


\section{Bibliography}

[1] K. Ziegler-Graham, E. J. MacKenzie, P. L. Ephraim, T. G. Travison, and R. Brookmeyer, "Estimating the Prevalence of Limb Loss in the United States: 2005 to 2050," Arch. Phys. Med. Rehabil., vol. 89, no. 3, pp. 422-429, Mar. 2008 .

[2] F. Cordella et al., "Literature review on needs of upper limb prosthesis users," Front. Neurosci., vol. 10, no. MAY, p. 209, May 2016.

[3] W. R. Frontera and J. K. Silver, Fondamenti di medicina fisica e riabilitativa. Verduci, 2004.

[4] M. Bianchi, R. Haschke, G. Büscher, S. Ciotti, N. Carbonaro, and A. Tognetti, "A multi-modal sensing glove for human manual-interaction studies," Electron., vol. 5, no. 3, Jul. 2016.

[5] S. G. Postema et al., "Musculoskeletal Complaints in Transverse Upper Limb Reduction Deficiency and Amputation in the Netherlands: Prevalence, Predictors, and Effect on Health," Arch. Phys. Med. Rehabil., vol. 97, no. 7, pp. 1137-1145, Jul. 2016.

[6] C. R. Gambrell, "Overuse syndrome and the unilateral upper limb amputee: Consequences and prevention," J. Prosthetics Orthot., vol. 20, no. 3, pp. 126132, Jul. 2008.

[7] L. E. Jones and J. H. Davidson, "Save that arm: A study of problems in the remaining arm of unilateral upper limb amputees," Prosthet. Orthot. Int., vol. 23, no. 1, pp. 55-58, Apr. 1999.

[8] K. Østlie, R. J. Franklin, O. H. Skjeldal, A. Skrondal, and P. Magnus, "Musculoskeletal pain and overuse syndromes in adult acquired major upperlimb amputees," Arch. Phys. Med. Rehabil., vol. 92, no. 12, pp. 1967-1973.e1, Dec. 2011. 
[9] E. Koskimies, N. Lindfors, M. Gissler, J. Peltonen, and Y. Nietosvaara, "Congenital upper limb deficiencies and associated malformations in Finland: A population-based study," J. Hand Surg. Am., vol. 36, no. 6, pp. 1058-1065, Jun. 2011.

[10] K. V. Ravindra et al., "Hand transplantation in the United States: Experience with 3 patients," Surgery, vol. 144, no. 4, pp. 638-644, Oct. 2008.

[11] J. M. Dubernard et al., "Human hand allograft: Report on first 6 months," Lancet, vol. 353, no. 9161, pp. 1315-1320, Apr. 1999.

[12] N. Alolabi, H. Augustine, and A. Thoma, "Hand transplantation: Current challenges and future prospects," Transplant Research and Risk Management, vol. 9. pp. 23-29, 2017.

[13] A. G. Nerlich, A. Zink, U. Szeimies, and H. G. Hagedorn, “Ancient Egyptian prosthesis of the big toe," Lancet, vol. 356, no. 9248. Elsevier, pp. 2176-2179, 23-Dec-2000.

[14] A. Otte, "3D Computer-Aided Design Reconstructions and 3D Multi-Material Polymer Replica Printings of the First 'Iron Hand' of Franconian Knight Gottfried (Götz) von Berlichingen (1480-1562): An Overview," Prosthesis, vol. 2, no. 4, pp. 304-312, Oct. 2020.

[15] A. Chadwell, N. Chinn, L. Kenney, Z. J. Karthaus, D. Mos, and G. Smit, “An evaluation of contralateral hand involvement in the operation of the Delft SelfGrasping Hand, an adjustable passive prosthesis," PLoS One, vol. 16, no. 6, p. e0252870, Jun. 2021.

[16] B. Maat, G. Smit, D. Plettenburg, and P. Breedveld, "Passive prosthetic hands and tools: A literature review," Prosthet. Orthot. Int., vol. 42, no. 1, pp. 66-74, Feb. 2018.

[17] L. F. Engels and C. Cipriani, "Nature's Masterpiece: How Scientists Struggle to Replace the Human Hand," Front. Young Minds, vol. 7, Jun. 2019.

[18] A. Grogan, “Artificial hand.,” Jun. 1906. 
[19] K. Ostlie, I. M. Lesjø, R. J. Franklin, B. Garfelt, O. H. Skjeldal, and P. Magnus, "Prosthesis use in adult acquired major upper-limb amputees: Patterns of wear, prosthetic skills and the actual use of prostheses in activities of daily life," Disabil. Rehabil. Assist. Technol., vol. 7, no. 6, pp. 479-493, Nov. 2012.

[20] Ottobock US, “Body-powered prosthetic solutions," 2021. [Online]. Available: https://www.ottobockus.com/prosthetics/upper-limb-prosthetics/solutionoverview/body-powered-prosthetic-solutions/. [Accessed: 16-Dec-2020].

[21] Enabling The Future, "WHICH DESIGN? - Enabling The Future," 2015. [Online]. Available: http://enablingthefuture.org/which-design/. [Accessed: 16-Dec-2020].

[22] J. M. Zuniga et al., "Functional changes through the usage of 3D-printed transitional prostheses in children," Disabil. Rehabil. Assist. Technol., vol. 14, no. 1, pp. 68-74, Jan. 2019.

[23] S. L. Carey, D. J. Lura, and M. Jason Highsmith, "Differences in myoelectric and body-powered upper-limb prostheses: Systematic literature review," $J$. Rehabil. Res. Dev., vol. 52, no. 3, pp. 247-262, 2015.

[24] S. B. Godfrey et al., "SoftHand at the CYBATHLON: A user's experience Olivier Lambercy; Roger Gassert," J. Neuroeng. Rehabil., vol. 14, no. 1, pp. 112, Nov. 2017.

[25] J. Billock, "Upper limb prosthetic terminal devices: Hands versus hooks," Clin. Prosthetics Orthot., vol. 10, no. 2, pp. 57-65, 1986.

[26] K. J. Zuo and J. L. Olson, "The evolution of functional hand replacement: From iron prostheses to hand transplantation," Canadian Journal of Plastic Surgery, vol. 22, no. 1. Pulsus Group Inc., pp. 44-51, 2014.

[27] E. D. SHERMAN, "a Russian Bioelectric-Controlled Prosthesis: Report of a Research Team From the Rehabilitation Institute of Montreal.," Can. Med. Assoc. J., vol. 91, no. 24, pp. 1268-1270, Dec. 1964.

[28] M. Rakić, “An automatic hand prosthesis," Med. Electron. Biol. Eng., vol. 2, no. 1, pp. 47-55, Mar. 1964. 
[29] M. Atzori, H. Müller, and H. Muller, "Control Capabilities of Myoelectric Robotic Prostheses by Hand Amputees: A Scientific Research and Market Overview," Front. Syst. Neurosci., vol. 9, no. Nov, p. 162, Nov. 2015.

[30] T. Feix, J. Romero, H. B. Schmiedmayer, A. M. Dollar, and D. Kragic, "The GRASP Taxonomy of Human Grasp Types," IEEE Trans. Human-Machine Syst., vol. 46, no. 1, pp. 66-77, Feb. 2016.

[31] M. G. Catalano, G. Grioli, E. Farnioli, A. Serio, C. Piazza, and A. Bicchi, "Adaptive synergies for the design and control of the Pisa/IIT SoftHand," Int. J. Rob. Res., vol. 33, no. 5, pp. 768-782, 2014.

[32] P. Weiner, J. Starke, F. Hundhausen, J. Beil, and T. Asfour, "The KIT Prosthetic Hand: Design and Control," in IEEE International Conference on Intelligent Robots and Systems, 2018, pp. 3328-3334.

[33] B. D. Adams, N. M. Grosland, D. M. Murphy, and M. Mccullough, "Impact of Impaired Wrist Motion on Hand and Upper-Extremity Performance,” 2003.

[34] F. Montagnani, M. Controzzi, and C. Cipriani, "Is it Finger or Wrist Dexterity That is Missing in Current Hand Prostheses?," IEEE Trans. Neural Syst. Rehabil. Eng., vol. 21, no. c, pp. 1-10, 2015.

[35] N. M. Bajaj, A. J. Spiers, and A. M. Dollar, "State of the Art in Artificial Wrists: A Review of Prosthetic and Robotic Wrist Design," IEEE Trans. Robot., vol. 35, no. 1, pp. 261-277, 2019.

[36] N. Jiang, S. Dosen, K. R. Muller, and D. Farina, "Myoelectric Control of Artificial Limbs: Is There a Need to Change Focus? [In the Spotlight]," IEEE Signal Process. Mag., vol. 29, no. 5, pp. 150-152, 2012.

[37] L. H. Smith, L. J. Hargrove, B. A. Lock, and T. A. Kuiken, "Determining the optimal window length for pattern recognition-based myoelectric control: Balancing the competing effects of classification error and controller delay," IEEE Trans. Neural Syst. Rehabil. Eng., vol. 19, no. 2, pp. 186-192, 2011. 
[38] A. Fougner, O. Stavdahl, P. J. Kyberd, Y. G. Losier, and P. A. Parker, "Control of upper limb prostheses: Terminology and proportional myoelectric controla review," IEEE Trans. Neural Syst. Rehabil. Eng., vol. 20, no. 5, pp. 663-677, 2012 .

[39] M. Hakonen, H. Piitulainen, and A. Visala, "Current state of digital signal processing in myoelectric interfaces and related applications," Biomed. Signal Process. Control, vol. 18, pp. 334-359, Apr. 2015.

[40] G. N. Saridis and T. P. Gootee, "EMG Pattern Analysis and Classification for a Prosthetic Arm," IEEE Trans. Biomed. Eng., vol. BME-29, no. 6, pp. 403412, Jun. 1982.

[41] S. Amsuess et al., "Context-dependent upper limb prosthesis control for natural and robust use," IEEE Trans. Neural Syst. Rehabil. Eng., vol. 24, no. 7, pp. 744-753, Jul. 2016.

[42] M. A. Oskoei and H. Hu, "Support vector machine-based classification scheme for myoelectric control applied to upper limb," IEEE Trans. Biomed. Eng., vol. 55, no. 8, pp. 1956-1965, Aug. 2008.

[43] M. Atzori, M. Cognolato, and H. Müller, "Deep learning with convolutional neural networks applied to electromyography data: A resource for the classification of movements for prosthetic hands," Front. Neurorobot., vol. 10, no. SEP, 2016.

[44] C. F. Pasluosta and A. W. L. Chiu, "Evaluation of a neural network-based control strategy for a cost-effective externally-powered prosthesis," Assist. Technol., vol. 24, no. 3, pp. 196-208, 2012.

[45] B. Karlik, M. O. Tokhi, and M. Alci, “A Fuzzy Clustering Neural Network Architecture for Multifunction Upper-Limb Prosthesis," IEEE Trans. Biomed. Eng., vol. 50, no. 11, pp. 1255-1261, 2003.

[46] D. Farina et al., "The extraction of neural information from the surface EMG for the control of upper-limb prostheses: Emerging avenues and challenges," IEEE Trans. Neural Syst. Rehabil. Eng., vol. 22, no. 4, pp. 797-809, Jul. 2014. 
[47] C. Igual, J. Igual, J. M. Hahne, and L. C. Parra, "Adaptive auto-regressive proportional myoelectric control," IEEE Trans. Neural Syst. Rehabil. Eng., vol. 27, no. 2, pp. 314-322, Feb. 2019.

[48] J. M. Hahne, M. A. Schweisfurth, M. Koppe, and D. Farina, "Simultaneous control of multiple functions of bionic hand prostheses: Performance and robustness in end users," Sci. Robot., vol. 3, no. 19, Jun. 2018.

[49] I. Kyranou, S. Vijayakumar, and M. S. Erden, "Causes of performance degradation in non-invasive electromyographic pattern recognition in upper limb prostheses," Front. Neurorobot., vol. 12, no. September, p. 58, Sep. 2018.

[50] G. Li, A. E. Schultz, and T. A. Kuiken, "Quantifying pattern recognition- based myoelectric control of multifunctional transradial prostheses," IEEE Trans. Neural Syst. Rehabil. Eng., vol. 18, no. 2, pp. 185-192, 2010.

[51] G. Emayavaramban, A. Amudha, T. Rajendran, M. Sivaramkumar, K. Balachandar, and T. Ramesh, "Identifying User Suitability in sEMG Based Hand Prosthesis Using Neural Networks," Curr. Signal Transduct. Ther., vol. 14, no. 2, pp. 158-164, Jun. 2018.

[52] S. Salminger et al., "Long-term implant of intramuscular sensors and nerve transfers for wireless control of robotic arms in above-elbow amputees," Sci. Robot., vol. 4, no. 32, p. 6306, Jul. 2019.

[53] E. N. Kamavuako, E. J. Scheme, and K. B. Englehart, "On the usability of intramuscular EMG for prosthetic control: A Fitts' Law approach," $J$. Electromyogr. Kinesiol., vol. 24, no. 5, pp. 770-777, Oct. 2014.

[54] S. M. Engdahl, B. P. Christie, B. Kelly, A. Davis, C. A. Chestek, and D. H. Gates, "Surveying the interest of individuals with upper limb loss in novel prosthetic control techniques," J. Neuroeng. Rehabil., vol. 12, no. 1, Jun. 2015.

[55] M. Ortiz-Catalan, R. Brånemark, B. Håkansson, and J. Delbeke, "On the viability of implantable electrodes for the natural control of artificial limbs: Review and discussion,” Biomed. Eng. Online, vol. 11, p. 33, Jun. 2012. 
[56] M. Jochumsen, A. Waris, and E. N. Kamavuako, "The effect of arm position on classification of hand gestures with intramuscular EMG," Biomed. Signal Process. Control, vol. 43, pp. 1-8, May 2018.

[57] D. Novak and R. Riener, "A survey of sensor fusion methods in wearable robotics," in Robotics and Autonomous Systems, 2015, vol. 73, pp. 155-170.

[58] R. C. Luo, C. C. Chang, and C. C. Lai, "Multisensor fusion and integration: Theories, applications, and its perspectives," IEEE Sens. J., vol. 11, no. 12, pp. 3122-3138, 2011.

[59] Y. Wu, D. Jiang, X. Liu, R. Bayford, and A. Demosthenous, "A HumanMachine Interface Using Electrical Impedance Tomography for Hand Prosthesis Control," IEEE Trans. Biomed. Circuits Syst., vol. 12, no. 6, pp. 1322-1333, Dec. 2018.

[60] R. Kusche and M. Ryschka, "Combining Bioimpedance and EMG Measurements for Reliable Muscle Contraction Detection," IEEE Sens. J., vol. 19, no. 23, pp. 11687-11696, Dec. 2019.

[61] W. Guo, X. Sheng, H. Liu, and X. Zhu, "Toward an Enhanced Human-Machine Interface for Upper-Limb Prosthesis Control with Combined EMG and NIRS Signals," IEEE Trans. Human-Machine Syst., vol. 47, no. 4, pp. 564-575, 2017.

[62] N. Akhlaghi et al., "Real-time classification of hand motions using ultrasound imaging of forearm muscles," IEEE Trans. Biomed. Eng., vol. 63, no. 8, pp. 1687-1698, Aug. 2016.

[63] X. Yang, J. Yan, Z. Chen, H. Ding, and H. Liu, "A proportional pattern recognition control scheme for wearable a-mode ultrasound sensing," IEEE Trans. Ind. Electron., vol. 67, no. 1, pp. 800-808, Jan. 2020.

[64] A. Fougner, E. Scheme, A. D. C. Chan, K. Englehart, and Ø. Stavdahl, "Resolving the Limb Position Effect in Myoelectric Pattern Recognition," IEEE Trans. Neural Syst. Rehabil. Eng., vol. 19, no. 6, pp. 644-651, 2011. 
[65] A. Gijsberts, M. Atzori, C. Castellini, H. Müller, and B. Caputo, "Movement error rate for evaluation of machine learning methods for sEMG-based hand movement classification," IEEE Trans. Neural Syst. Rehabil. Eng., vol. 22, no. 4, 2014.

[66] A. Krasoulis, I. Kyranou, M. S. Erden, K. Nazarpour, and S. Vijayakumar, "Improved prosthetic hand control with concurrent use of myoelectric and inertial measurements," J. Neuroeng. Rehabil., vol. 14, no. 1, Jul. 2017.

[67] D. A. Bennett and M. Goldfarb, "IMU-Based Wrist Rotation Control of a Transradial Myoelectric Prosthesis," IEEE Trans. Neural Syst. Rehabil. Eng., vol. 26, no. 2, pp. 419-427, 2018.

[68] D. Johansen, C. Cipriani, D. B. Popovic, and L. N. S. A. Struijk, "Control of a Robotic Hand Using a Tongue Control System-A Prosthesis Application," IEEE Trans. Biomed. Eng., vol. 63, no. 7, pp. 1368-1376, 2016.

[69] J. Gonzalez-Vargas, S. Dosen, S. Amsuess, W. Yu, and D. Farina, "HumanMachine Interface for the Control of Multi-Function Systems Based on Electrocutaneous Menu: Application to Multi- Grasp Prosthetic Hands,” 2015.

[70] Y. Hao et al., "Controlling hand-assistive devices: Utilizing electrooculography as a substitute for vision," IEEE Robot. Autom. Mag., vol. 20, no. 1, pp. 40-52, Mar. 2013.

[71] M. M. D. Sobuh et al., "Visuomotor behaviours when using a myoelectric prosthesis," J. Neuroeng. Rehabil., vol. 11, no. 1, p. 72, Apr. 2014.

[72] M. Liu, D. Wang, and H. Helen Huang, "Development of an EnvironmentAware Locomotion Mode Recognition System for Powered Lower Limb Prostheses," IEEE Trans. Neural Syst. Rehabil. Eng., vol. 24, no. 4, pp. 434443, Apr. 2016.

[73] T. Carlson and Y. Demiris, "Collaborative control for a robotic wheelchair: Evaluation of performance, attention, and workload," IEEE Trans. Syst. Man, Cybern. Part B Cybern., vol. 42, no. 3, pp. 876-888, 2012. 
[74] I. M. Mitchell, P. Viswanathan, B. Adhikari, E. Rothfels, and A. K. Mackworth, "Shared control policies for safe wheelchair navigation of elderly adults with cognitive and mobility impairments: Designing a wizard of oz study," in Proceedings of the American Control Conference, 2014, pp. 4087-4094.

[75] M. Markovic, S. Dosen, C. Cipriani, D. Popovic, and D. Farina, "Stereovision and augmented reality for closed-loop control of grasping in hand prostheses," J. Neural Eng., vol. 11, no. 4, p. 046001, Aug. 2014.

[76] Y. He, R. Kubozono, O. Fukuda, N. Yamaguchi, and H. Okumura, "VisionBased Assistance for Myoelectric Hand Control," IEEE Access, vol. 8, pp. 201956-201965, 2020.

[77] J. DeGol, A. Akhtar, B. Manja, and T. Bretl, “Automatic grasp selection using a camera in a hand prosthesis," in 2016 38th Annual International Conference of the IEEE Engineering in Medicine and Biology Society (EMBC), 2016, vol. 2016-Octob, pp. 431-434.

[78] G. Ghazaei, A. Alameer, P. Degenaar, G. Morgan, and K. Nazarpour, "Deep learning-based artificial vision for grasp classification in myoelectric hands," $J$. Neural Eng., vol. 14, no. 3, p. 036025, Jun. 2017.

[79] J. Fajardo, V. Ferman, A. Munoz, D. Andrade, A. R. Neto, and E. Rohmer, "User-Prosthesis Interface for Upper Limb Prosthesis Based on Object Classification," in 2018 Latin American Robotic Symposium, 2018 Brazilian Symposium on Robotics (SBR) and 2018 Workshop on Robotics in Education (WRE), 2018, pp. 390-395.

[80] S. Došen, C. Cipriani, M. Kostić, M. Controzzi, M. C. Carrozza, and D. B. Popovič, "Cognitive vision system for control of dexterous prosthetic hands: Experimental evaluation," J. Neuroeng. Rehabil., vol. 7, no. 1, p. 42, Jan. 2010.

[81] M. Markovic, S. Dosen, D. Popovic, B. Graimann, and D. Farina, "Sensor fusion and computer vision for context-aware control of a multi degree-offreedom prosthesis.," J. Neural Eng., vol. 12, no. 6, p. 066022, Nov. 2015. 
[82] J. Li, C. Shi, D. Yang, and H. Liu, "A novel grasping control method for dexterous prosthesis based on eye-tracking," IEEE Int. Conf. Robot. Biomimetics, ROBIO 2019, pp. 2293-2297, Dec. 2019.

[83] C. Shi, L. Qi, D. Yang, J. Zhao, and H. Liu, "A novel method of combining computer vision, eye-tracking, EMG, and IMU to control dexterous prosthetic hand," in IEEE International Conference on Robotics and Biomimetics, ROBIO $2019,2019$.

[84] N. E. Krausz, D. Lamotte, I. Batzianoulis, L. J. Hargrove, S. Micera, and A. Billard, "Intent Prediction Based on Biomechanical Coordination of EMG and Vision-Filtered Gaze for End-Point Control of an Arm Prosthesis," IEEE Trans. Neural Syst. Rehabil. Eng., vol. 28, no. 6, pp. 1471-1480, Jun. 2020.

[85] D. P. McMullen et al., "Demonstration of a Semi-Autonomous Hybrid BrainMachine Interface Using Human Intracranial EEG, Eye Tracking, and Computer Vision to Control a Robotic Upper Limb Prosthetic," IEEE Trans. Neural Syst. Rehabil. Eng., vol. 22, no. 4, pp. 784-796, Jul. 2014.

[86] D. A. Rosenbaum, C. M. Van Heugten, and G. E. Caldwell, "From cognition to biomechanics and back: The end-state comfort effect and the middle-is-faster effect," Acta Psychol. (Amst)., vol. 94, no. 1, pp. 59-85, Oct. 1996.

[87] O. Herbort, A. Koning, J. van Uem, and R. G.J. Meulenbroek, "The end-state comfort effect facilitates joint action," Acta Psychol. (Amst)., vol. 139, no. 3, pp. 404-416, 2012.

[88] F. Hundhausen, D. Megerle, and T. Asfour, "Resource-Aware Object Classification and Segmentation for Semi-Autonomous Grasping with Prosthetic Hands," in IEEE-RAS International Conference on Humanoid Robots, 2019, vol. 2019-Octob, pp. 215-221.

[89] Y. He, R. Shima, O. Fukuda, N. Bu, N. Yamaguchi, and H. Okumura, "Development of distributed control system for vision-based myoelectric prosthetic hand," IEEE Access, vol. 7, pp. 54542-54549, 2019. 
[90] A. Chadwell et al., "Upper limb activity in myoelectric prosthesis users is biased towards the intact limb and appears unrelated to goal-directed task performance," Sci. Rep., vol. 8, no. 1, 2018.

[91] A. Mingrino, A. Bucci, R. Magni, and P. Dario, "Slippage control in hand prostheses by sensing grasping forces and sliding motion," in IEEE/RSJ/GI International Conference on Intelligent Robots and Systems, 1994, vol. 3, pp. 1803-1809.

[92] G. Puchhammer, "The tactile slip sensor: integration of a min- iaturized sensory device on an myoelectric hand," Orthopadie-Technik Quart, vol. 1, no. 2000, pp. 7-12, 2000.

[93] E. D. Engeberg and S. G. Meek, "Adaptive sliding mode control for prosthetic hands to simultaneously prevent slip and minimize deformation of grasped objects," IEEE/ASME Trans. Mechatronics, vol. 18, no. 1, pp. 376-385, Feb. 2013

[94] Z. Ray and E. D. Engeberg, "Human-inspired reflex to autonomously prevent slip of grasped objects rotated with a prosthetic hand," J. Healthc. Eng., vol. 2018, 2018.

[95] K. Z. Zhuang et al., "Shared human-robot proportional control of a dexterous myoelectric prosthesis," Nat. Mach. Intell., vol. 1, no. 9, pp. 400-411, Sep. 2019.

[96] M. Tavakoli et al., "Autonomous Selection of Closing Posture of a Robotic Hand Through Embodied Soft Matter Capacitive Sensors," IEEE Sens. J., vol. 17, no. 17, pp. 5669-5677, Sep. 2017.

[97] M. Carrasco and X. Clady, "Exploiting eye-hand coordination to detect grasping movements," Image Vis. Comput., vol. 30, no. 11, pp. 860-874, Nov. 2012

[98] B. Zhong, H. Huang, and E. Lobaton, "Reliable Vision-Based Grasping Target Recognition for Upper Limb Prostheses," IEEE Trans. Cybern., pp. 1-13, Jun. 2020 . 
[99] F. Montagnani, M. Controzzi, and C. Cipriani, "Exploiting arm posture synergies in activities of daily living to control the wrist rotation in upper limb prostheses: A feasibility study," in Proceedings of the Annual International Conference of the IEEE Engineering in Medicine and Biology Society, EMBS, 2015, vol. 2015-November, pp. 2462-2465.

[100] S. L. Carey, M. Jason Highsmith, M. E. Maitland, and R. V. Dubey, "Compensatory movements of transradial prosthesis users during common tasks," Clin. Biomech., vol. 23, no. 9, pp. 1128-1135, Nov. 2008.

[101] R. Volkmar, S. Dosen, J. Gonzalez-Vargas, M. Baum, and M. Markovic, "Improving bimanual interaction with a prosthesis using semi-autonomous control," J. Neuroeng. Rehabil., vol. 16, no. 1, pp. 1-13, Nov. 2019.

[102] F. Flemisch, D. Abbink, M. Itoh, M. P. Pacaux-Lemoine, and G. Weßel, "Shared control is the sharp end of cooperation: Towards a common framework of joint action, shared control and human machine cooperation," IFACPapersOnLine, vol. 49, no. 19, pp. 72-77, 2016.

[103] N. Amirshirzad, A. Kumru, and E. Oztop, "Human adaptation to human-robot shared control," IEEE Trans. Human-Machine Syst., vol. 49, no. 2, pp. 126136, Apr. 2019.

[104] A. Cherubini and D. Navarro-Alarcon, "Sensor-Based Control for Collaborative Robots: Fundamentals, Challenges, and Opportunities," Front. Neurorobot., vol. 14, p. 113, Jan. 2021.

[105] C. Sherstan, J. Modayil, and P. M. Pilarski, "A Collaborative Approach to the Simultaneous Multi-joint Control of a Prosthetic Arm," in IEEE International Conference on Rehabilitation Robotics, 2015, vol. 2015-September, pp. 13-18.

[106] T. Feix, I. M. Bullock, and A. M. Dollar, "Analysis of human grasping behavior: Object characteristics and grasp type," IEEE Trans. Haptics, vol. 7, no. 3, pp. 311-323, Jul. 2014.

[107] F. Cini, V. Ortenzi, P. Corke, and M. Controzzi, "On the choice of grasp type and location when handing over an object," 2019. 
[108] C. Shi, D. Yang, J. Zhao, and H. Liu, “Computer Vision-Based Grasp Pattern Recognition with Application to Myoelectric Control of Dexterous Hand Prosthesis," IEEE Trans. Neural Syst. Rehabil. Eng., vol. 28, no. 9, pp. 20902099, 2020.

[109] R. Jonker and T. Volgenant, "Improving the Hungarian assignment algorithm," Oper. Res. Lett., vol. 5, no. 4, pp. 171-175, Oct. 1986.

[110] R. B. Rusu and S. Cousins, "3D is here: Point Cloud Library (PCL)," in Proceedings - IEEE International Conference on Robotics and Automation, 2011.

[111] M. A. Fischler and R. C. Bolles, "Random sample consensus: A Paradigm for Model Fitting with Applications to Image Analysis and Automated Cartography," Commun. ACM, vol. 24, no. 6, pp. 381-395, Jun. 1981.

[112] J. Papon, A. Abramov, M. Schoeler, and F. Worgotter, "Voxel cloud connectivity segmentation - Supervoxels for point clouds," in Proceedings of the IEEE Computer Society Conference on Computer Vision and Pattern Recognition, 2013, pp. 2027-2034.

[113] G. Gao, M. Lauri, J. Zhang, and S. Frintrop, "Saliency-guided adaptive seeding for supervoxel segmentation," IEEE Int. Conf. Intell. Robot. Syst., vol. 2017Septe, pp. 4938-4943, Dec. 2017.

[114] S. C. Stein, M. Schoeler, J. Papon, and F. Worgotter, "Object partitioning using local convexity," in Proceedings of the IEEE Computer Society Conference on Computer Vision and Pattern Recognition, 2014, pp. 304-311.

[115] W. Vogt, “Mahalanobis Distance,” 1999.

[116] M. Carfagni, R. Furferi, L. Governi, M. Servi, F. Uccheddu, and Y. Volpe, “On the Performance of the Intel SR300 Depth Camera: Metrological and Critical Characterization," IEEE Sens. J., vol. 17, no. 14, pp. 4508-4519, Jul. 2017.

[117] J. Linde, "Marker-based Hand Tracking for Prosthesis Control," Georg-August University Göttingen, 2017. 
[118] "Leica Camera AG \& pmd Expand Partnership with New 3D Camera." [Online]. Available: https://pmdtec.com/news_media/press_release/leicapmd.php. [Accessed: 29-Jan-2020].

[119] H. Choudhry and S. Khan, "smartARM | Supercharged bionics." [Online]. Available: https://smartarm.ca/. [Accessed: 10-Sep-2019].

[120] "Why Would Prosthetic Arms Need to See or Connect to Cloud AI? | Microsoft Docs.” [Online]. Available: https://docs.microsoft.com/frfr/archive/blogs/machinelearning/why-would-prosthetic-arms-need-to-see-orconnect-to-cloud-ai?utm_source=vs_developer_news\&utm_medium=referral. [Accessed: 18-Feb-2020].

[121] R. Mur-Artal, J. M. M. Montiel, and J. D. Tardos, “ORB-SLAM: A Versatile and Accurate Monocular SLAM System," IEEE Trans. Robot., vol. 31, no. 5, pp. 1147-1163, 2015.

[122] R. Mur-Artal and J. D. Tardos, "ORB-SLAM2: An Open-Source SLAM System for Monocular, Stereo, and RGB-D Cameras," IEEE Trans. Robot., vol. 33, no. 5, pp. 1255-1262, 2017.

[123] F. Teich, T. Lüddecke, and F. Wörgötter, “3D object classification via part graphs," VISIGRAPP 2021 - Proc. 16th Int. Jt. Conf. Comput. Vision, Imaging Comput. Graph. Theory Appl., vol. 5, pp. 417-426, 2021.

[124] J. Mouchoux, S. Carisi, S. Dosen, D. Farina, A. F. Schilling, and M. Markovic, "Artificial Perception and Semiautonomous Control in Myoelectric Hand Prostheses Increases Performance and Decreases Effort," IEEE Trans. Robot., vol. 37, no. 4, pp. 1298-1312, 2021.

[125] S. Wang et al., "Evaluation of Performance-Based Outcome Measures for the Upper Limb: A Comprehensive Narrative Review," PM R, vol. 10, no. 9, pp. 951-962.e3, Sep. 2018.

[126] C. M. Light, P. H. Chappell, and P. J. Kyberd, "Establishing a standardized clinical assessment tool of pathologic and prosthetic hand function: Normative data, reliability, and validity," Arch. Phys. Med. Rehabil., vol. 83, no. 6, pp. 776-783, Jun. 2002. 
[127] "Focals by North." [Online]. Available: https://www.bynorth.com/. [Accessed: 16-Nov-2019].

[128] D. P. Losey, C. G. McDonald, E. Battaglia, and M. K. O’Malley, “A review of intent detection, arbitration, and communication aspects of shared control for physical human-robot interaction," Applied Mechanics Reviews, vol. 70, no. 1. American Society of Mechanical Engineers (ASME), 01-Jan-2018.

[129] T. Carlson and J. Del R. Millan, "Brain-controlled wheelchairs: A robotic architecture," IEEE Robot. Autom. Mag., vol. 20, no. 1, pp. 65-73, 2013.

[130] K. Stojmenova and J. Sodnik, "Detection-response task - uses and limitations," Sensors (Switzerland), vol. 18, no. 2, Feb. 2018.

[131] P. Viswanathan et al., "A Wizard-of-Oz Intelligent Wheelchair Study with Cognitively- Impaired Older Adults: Attitudes toward User Control,” in IEEE/RSJ International Conference on Intelligent Robots and Systems, 2014.

[132] A. Kadambi, A. Bhandari, and R. Raskar, "3D depth cameras in vision: Benefits and limitations of the hardware with an emphasis on the first-and secondgeneration kinect models," in Advances in Computer Vision and Pattern Recognition, vol. 67, Springer London, 2014, pp. 3-26.

[133] M. S. Ahn, H. Chae, D. Noh, H. Nam, and D. Hong, "Analysis and Noise Modeling of the Intel RealSense D435 for Mobile Robots," in 2019 16th International Conference on Ubiquitous Robots, UR 2019, 2019, pp. 707-711.

[134] T. Mallick, P. P. Das, and A. K. Majumdar, "Characterizations of noise in Kinect depth images: A review," IEEE Sensors Journal, vol. 14, no. 6. Institute of Electrical and Electronics Engineers Inc., pp. 1731-1740, 2014.

[135] D. Strazdas, J. Hintz, A. M. Felbberg, and A. Al-Hamadi, "Robots and Wizards: An Investigation into Natural Human-Robot Interaction," IEEE Access, vol. 8, pp. 207635-207642, 2020.

[136] M. Jeannerod, "The timing of natural prehension movements," J. Mot. Behav., vol. 16, no. 3, pp. 235-254, Sep. 1984. 
[137] M. Jeannerod, "Visuomotor channels: Their integration in goal-directed prehension-," Hum. Mov. Sci., vol. 18, no. 2-3, pp. 201-218, Jun. 1999.

[138] T. R. Farrell and R. F. Weir, "The optimal controller delay for myoelectric prostheses," IEEE Trans. Neural Syst. Rehabil. Eng., vol. 15, no. 1, pp. 111118, Mar. 2007.

[139] M. Tsakiris and P. Haggard, Experimenting with the acting self, vol. 22, no. 34. Psychology Press, 2005, pp. 387-407.

[140] H. Grea, M. Desmurget, and C. Prablanc, "Postural invariance in threedimensional reaching and grasping movements," Exp. Brain Res., vol. 134, no. 2, pp. 155-162, Sep. 2000.

[141] M. Santello, M. Flanders, and J. F. Soechting, "Postural hand synergies for tool use," J. Neurosci., vol. 18, no. 23, pp. 10105-10115, Dec. 1998.

[142] J. Starke, C. Eichmann, S. Ottenhaus, and T. Asfour, "Synergy-Based, DataDriven Generation of Object-Specific Grasps for Anthropomorphic Hands," in IEEE-RAS International Conference on Humanoid Robots, 2019, vol. 2018Novem, pp. 327-333.

[143] J. Starke, C. Eichmann, S. Ottenhaus, and T. Asfour, "Human-Inspired Representation of Object-Specific Grasps for Anthropomorphic Hands," Int. J. Humanoid Robot., vol. 17, no. 2, Apr. 2020.

[144] M. Lotze, W. Grodd, N. Birbaumer, M. Erb, E. Huse, and H. Flor, "Does use of a myoelectric prosthesis prevent cortical reorganization and phantom limb pain? [2]," Nat. Neurosci., vol. 2, no. 6, pp. 501-502, Jun. 1999.

[145] G. Gorisse, O. Christmann, E. A. Amato, and S. Richir, "First- and ThirdPerson Perspectives in Immersive Virtual Environments: Presence and Performance Analysis of Embodied Users," Front. Robot. AI, vol. 4, p. 33, Jul. 2017.

[146] O. Blanke and T. Metzinger, "Full-body illusions and minimal phenomenal selfhood," Trends Cogn. Sci., vol. 13, no. 1, pp. 7-13, Jan. 2009. 
[147] K. Kilteni, R. Groten, and M. Slater, "The Sense of embodiment in virtual reality," Presence: Teleoperators and Virtual Environments, vol. 22, no. 1. pp. 373-387, 2013.

[148] H. H. Ehrsson, B. Rosén, A. Stockselius, C. Ragnö, P. Köhler, and G. Lundborg, "Upper limb amputees can be induced to experience a rubber hand as their own," Brain, vol. 131, no. 12, pp. 3443-3452, Dec. 2008.

[149] K. L. Collins, A. Guterstam, J. Cronin, J. D. Olson, H. H. Ehrsson, and J. G. Ojemann, "Ownership of an artificial limb induced by electrical brain stimulation," Proc. Natl. Acad. Sci. U. S. A., vol. 114, no. 1, pp. 166-171, Jan. 2017.

[150] J. W. Sensinger and S. Dosen, “A Review of Sensory Feedback in Upper-Limb Prostheses From the Perspective of Human Motor Control," Front. Neurosci., vol. 14 , p. 345 , Jun. 2020.

[151] J. Zbinden and M. Ortiz-Catalan, "The rubber hand illusion is a fallible method to study ownership of prosthetic limbs," Sci. Rep., vol. 11, no. 1, pp. 1-11, Feb. 2021.

[152] P. Haggard, S. Clark, and J. Kalogeras, "Voluntary action and conscious awareness," Nat. Neurosci., vol. 5, no. 4, pp. 382-385, 2002.

[153] J. Arata, M. Hattori, S. Ichikawa, and M. Sakaguchi, "Robotically enhanced rubber hand illusion," IEEE Trans. Haptics, vol. 7, no. 4, pp. 526-532, Oct. 2014. 



\title{
Curriculum Vitae - Jérémy Mouchoux
}

\author{
Address HainbundStr. 21 \\ 37085 Göttingen \\ Nationality French \\ Date of Birth $16^{\text {th }}$ March 1993 \\ Place of Birth $\quad \mathrm{S}^{\mathrm{t}}$ Cyr l'École, FRANCE \\ Email jeremymouchoux@msn.com
}

\section{$\underline{\text { Education/Qualifications }}$}

2017-2021: Ph.D. Student at the GAUSS university in Göttingen, GERMANY, working at the Universitätsmedizin Göttingen (UMG):

Design and development of a semi-autonomous context-aware upper limb prosthesis using computer vision, IMUs, data fusion, and machine learning

2013-2016: Student in general engineering at Ecole Centrale de Nantes (a highly selective French School of Engineering conferring a diploma equivalent to a Master's Degree), Nantes FRANCE:

Specializations :

- Signal treatment for medical applications

- Management and communication

- Virtual reality

2011-2013: Classes préparatoire (a 2-year intensive program preparing for the national competitive exams for entry to French Schools of Engineering) at Chaptal, Paris FRANCE:

- 2012-2013: Physic and Industrial Science

- 2011-2013: Mathematics, Physics, and Industrial Science

2011: Baccalaureate (equivalent to A levels) in Science

\section{$\underline{\text { Work Experience }}$}

\begin{tabular}{|c|c|}
\hline $\begin{array}{l}\text { Mai } 2017 \text { to } \\
\text { October } 2021\end{array}$ & $\begin{array}{l}\text { Research assistant in the Applied Rehabilitation Technology } \\
\text { Laboratory (ART-Lab), Department for Trauma surgery, } \\
\text { Orthopedics, and Plastic Surgery (CUOP) } \\
\text { University Medical Center Göttingen } \\
\text { Work financed by the German Ministry for Education and } \\
\text { Research (BMBF) under the project INOPRO (16SV7657). }\end{array}$ \\
\hline
\end{tabular}




\begin{tabular}{|c|c|}
\hline $\begin{array}{c}\text { February 2017 to } \\
\text { Mai 2017: }\end{array}$ & $\begin{array}{c}\text { Informatic provider in Astek company working at IXBlue } \\
\text { company on PASSION project: } \\
\text { Development of a new military navigation software with } \\
\text { sensor data fusion and augmented reality. }\end{array}$ \\
\hline $\begin{array}{c}\text { April 2016 to } \\
\text { September 2016: }\end{array}$ & $\begin{array}{c}\text { Internship at the neurorehabilitation department of the } \\
\text { Medical University of Göttingen, GERMANY. } \\
\text { Conception of an autonomous prosthesis using depth camera } \\
\text { and augmented reality feedback }\end{array}$ \\
\hline $\begin{array}{c}\text { March 2015 to } \\
\text { August 2015: }\end{array}$ & $\begin{array}{c}\text { Internship at Light-and-Shadows, Suresnes FRANCE: } \\
\text { Developed a virtual mannequin tracked in real-time on Unity } \\
\text { software. Participated in the design of interactive devices. }\end{array}$ \\
\hline July 2014: & $\begin{array}{c}\text { Internship in a Siniat's Factory, Chateau Gontier, FRANCE } \\
\text { Study and optimization of the production chain. }\end{array}$ \\
\hline $\begin{array}{c}\text { February 2014 to } \\
\text { June 2014: }\end{array}$ & $\begin{array}{c}\text { Industrial Study Project with Cezus-Areva, Paimboeuf } \\
\text { FRAn } \\
\text { Optimization of a tube cutting machine in a team of students. }\end{array}$ \\
\hline
\end{tabular}

\section{$\underline{\text { Publications }}$}

Mouchoux, Jeremy, Carisi, S., Dosen, S., Farina, D., Schilling, A. F., \& Markovic, M. (2021). Artificial Perception and Semiautonomous Control in Myoelectric Hand Prostheses Increases Performance and Decreases Effort. IEEE Transactions on Robotics. https://doi.org/10.1109/TRO.2020.3047013

Mouchoux, Jérémy, Bravo-Cabrera, M. A., Dosen, S., Schilling, A. F., \& Markovic, M. (2021). Impact of Shared Control Modalities on Performance and Usability of Semi-autonomous Prostheses. Frontiers in Neurorobotics, 0, 172. https://doi.org/10.3389/FNBOT.2021.768619

\section{Conferences and Posters}

J. Mouchoux, S. Carisi, and M. Markovic, "Intelligent, context-aware myoelectric hand prosthesis", Deutscher Kongress für Orthopädie und Unfallchirurgie (DKOU 2018). Berlin, 23.-26.10.2018. Düsseldorf: German Medical Science GMS Publishing House; 2018. DocPT11-1003

(Poster)

J. Mouchoux, A. F. Schilling, and M. Markovic, "Task decomposition as a new approach for assessing the prosthesis performance in standardized tests", In CYBATHLON Arm and Leg Prosthesis Series \& Symposium on Assistive and Wearable Robotics, AsWeR 2019.

(Presentation)

\section{Prizes}

Second price for the clinical poster "Intelligent, context-aware myoelectric hand prosthesis" at DKOU 2018 